\title{
Higher cluster categories and QFT dualities
}

\author{
Sebastián Franco ${ }^{1,2, *}$ and Gregg Musiker ${ }^{3, \dagger}$ \\ ${ }^{1}$ Physics Department, The City College of the CUNY 160 Convent Avenue, \\ New York, New York 10031, USA \\ ${ }^{2}$ The Graduate School and University Center, \\ The City University of New York 365 Fifth Avenue, New York, New York 10016, USA \\ ${ }^{3}$ School of Mathematics, University of Minnesota, Minneapolis, Minnesota 55455, USA
}

(Received 22 June 2018; published 27 August 2018)

\begin{abstract}
We introduce a unified mathematical framework that elegantly describes minimally supersymmetry gauge theories in even dimensions, ranging from six dimensions to zero dimensions, and their dualities. This approach combines and extends recent developments on graded quivers with potentials, higher Ginzburg algebras, and higher cluster categories (also known as $m$-cluster categories). Quiver mutations studied in the context of mathematics precisely correspond to the order- $(m+1)$ dualities of the gauge theories. Our work indicates that these equivalences of quiver gauge theories sit inside an infinite family of such generalized dualities.
\end{abstract}

DOI: 10.1103/PhysRevD.98.046021

\section{INTRODUCTION}

Recently, it was realized that minimally supersymmetric gauge theories in $6-2 m$ dimensions exhibit order- $(m+1)$ dualities, generalizing the well-known case of Seiberg duality for four-dimensional (4D) $\mathcal{N}=1$ theories [1]. ${ }^{1}$ The first hint in this direction was the discovery that twodimensional (2D) $\mathcal{N}=(0,2)$ gauge theories enjoy an order3 duality named triality [2]. This was soon followed by the proposal of quadrality, an order-4 duality, for zero-dimensional (0D) $\mathcal{N}=1$ gauge theories [3].

There has also been significant progress in the brane engineering of $2 \mathrm{D} \mathcal{N}=(0,2)$ and $0 d \mathcal{N}=1$ theories. These constructions include D-brane probes of toric CalabiYau (CY) singularities [4], T-dual brane configurations generalizing brane tilings [5-8] and D-branes in the mirror geometries $[3,9] .^{2}$ These brane configurations have been useful for both understanding and postulating some of these dualities.

\footnotetext{
*sfranco@ccny.cuny.edu

musiker@math.umn.edu

${ }^{1}$ By "order- $(m+1)$ duality," we mean a generalization of duality relating $(m+1)$ different theories. Furthermore, in this case, $(m+1)$ consecutive applications of an elementary duality transformation amount to the identity.

${ }^{2}$ See Refs. [10-13] for alternative constructions of $2 \mathrm{D}$ $\mathcal{N}=(0,2)$ theories.

Published by the American Physical Society under the terms of the Creative Commons Attribution 4.0 International license. Further distribution of this work must maintain attribution to the author(s) and the published article's title, journal citation, and DOI. Funded by SCOAP ${ }^{3}$.
}

In parallel, there have been interesting mathematical developments concerning graded quivers with potentials $[14,15]$, higher Ginzburg algebras [15,16], and higher cluster categories [14]. While these topics are closely related to each other, their presentation in the literature has not been fully integrated. In this paper, we will show that they can be combined into a unified mathematical framework that elegantly describes minimally supersymmetric (SUSY) gauge theories in even dimensions, ranging from six dimensions to zero dimensions. Moreover, quiver mutations studied in the mathematical context precisely correspond to the order- $(m+1)$ dualities of the gauge theories. Higher Ginzburg algebras thus provide an algebraic unification of gauge theories in different dimensions and their dualities, which is similar to the geometric unification attained in Refs. [3,9,17] using mirror symmetry. Interestingly, this realization implies that these equivalences of quiver gauge theories sit inside an infinite family of such generalized dualities. For general $m$, the graded quivers and their new dualities have a physical realization in terms of the category of branes in the topological B-model on CY $(m+2)$-folds [18]. Therefore, our framework significantly extends the results beyond those for six-dimensional (6D) to OD gauge theories. Our presentation will try to make the mathematical concepts accessible to the physics audience and vice versa.

This paper is organized as follows. Section II introduces graded quivers with potentials. These are quivers containing different types of arrows, the number of which is controlled by an integer $m \geq 1$. Section III discusses the higher Ginzburg algebras associated to such quivers. Section IV contains some of the key ideas of this paper, introducing 
mutations of graded quivers and their potentials. The order of such mutations is established in Sec. V. In Sec. VI, the field theoretic concept of anomalies is generalized to graded quivers with arbitrary $m$. Section VII outlines the connection between graded quivers with $m \leq 3$ and physics, in terms of gauge theories in various dimensions and $\mathrm{D}$-brane probing CY singularities. Section VIII discusses at length the connection between graded quivers with $m=0,1,2,3$ and minimally supersymmetric gauge theories in $d=6,4$, 2,0 . Section IX explains how the mutations of graded quivers unify the order- $(m+1)$ dualities of the corresponding gauge theories. In Sec. X, we discuss the class of graded quivers coming from toric CY's and explain how they are described using mirror symmetry. Section XI generalizes the physical notion of dimensional reduction to arbitrary $m$. We summarize our results and present directions for future research in Sec. XII. We also include four Appendices, discussing the mathematics of potentials, the mutation of differentials, cluster categories, and silting.

\section{GRADED QUIVERS WITH POTENTIALS}

In this section, we introduce graded quivers with potentials. Our treatment combines the ideas developed in Refs. [14,15]. Buan and Thomas [14] defined graded quivers, called colored quivers therein, motivated by their generalization of cluster categories to higher cluster categories (or $m$-cluster categories). Further mathematical details of (higher) cluster categories and (higher) tilting theory are included in Appendix C. Oppermann [15] was motivated by a variant of tilting theory known as silting, see Appendix D, and higher Ginzburg algebras. Because of its closer connection to Calabi-Yau manifolds and physics, we utilize this second perspective for the majority of this paper.

\section{A. Graded quivers}

A quiver $Q=\left(Q_{0}, Q_{1}, s, t\right)$ consists of a set of nodes $Q_{0},{ }^{3}$ a set of arrows $Q_{1}$, and two functions $s$ and $t$ that denote the start and target of every arrow. In particular, $\varphi: v \rightarrow w \in Q_{1}$ has $s(\varphi)=v$ and $t(\varphi)=w$. We say that a quiver is finite if it consists of a finite number of nodes and arrows. A path is a concatenation of arrows $\varphi_{1} \varphi_{2} \cdots \varphi_{k}$ such that $s\left(\varphi_{i+1}\right)=t\left(\varphi_{i}\right)$. We say that $k$ is the length of such a path. A path is known as a cycle if in addition it satisfies the identity $s\left(\varphi_{1}\right)=t\left(\varphi_{k}\right)$.

Given an algebraically closed field $k$, e.g., $\mathbb{C}$, we let $k Q$ be the path algebra of $Q$. The path algebra is defined as the algebra of which the elements are paths plus idempotents $e_{i}$ for $i \in Q_{0}$. By convention, we consider $e_{i}$ to be a path of length 0 and set $s\left(e_{i}\right)=t\left(e_{i}\right)=i$. We define multiplication in the path algebra by concatenation, i.e., $p \cdot q=p q$ if $s(q)=t(p)$ and $p \cdot q=0$ otherwise (where $p$ or $q=e_{i}$ possible). In particular, $e_{i} \cdot e_{j}=\delta_{i j} e_{i}$, i.e., the $\left\{e_{i}\right\}$ 's

\footnotetext{
${ }^{3}$ The nodes are often indexed as $\{1,2, \ldots, n\}$.
}

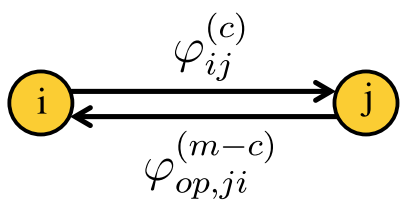

FIG. 1. Double arrow in a graded quiver.

indeed form an orthogonal collection of elements that are unchanged by taking their power.

We now fix $m$ to be a nonnegative integer and use this parameter to turn $Q$ into a graded quiver $\bar{Q}$. In particular, $\overline{Q_{0}}=$ $Q_{0}$ is the same set of nodes, but $\overline{Q_{1}}$ is now the set of graded arrows. A graded arrow $\varphi: i \rightarrow j \in Q_{1}$ has a start $s(\varphi)=i \in Q_{0}$, a target $t(\varphi)=j \in Q_{0}$, and a degree $|\varphi|$, which we will assume is an integer from the set $\{0,1,2, \ldots, m\}{ }^{4}$ For every graded arrow $\varphi \in Q_{1}$, we also adjoin its opposite $\varphi_{\mathrm{op}}: j \rightarrow i$ with its start and target reversed and degree given as $\left|\varphi_{\text {op }}\right|=m-|\varphi|$. Since the integer $m$ determines the possible degrees or colors, different values of $m$ give rise to qualitatively different classes of graded quivers.

Lastly, for every node $i \in Q_{0}$, we adjoin a loop $\ell_{i}$ based at node $i$, i.e., with $s\left(\ell_{i}\right)=t\left(\ell_{i}\right)=i$ and degree $\left|\ell_{i}\right|=m+1$. These special loops are the only arrows of $\bar{Q}$ with degree greater than $m$. Since such loops are present at every node, we will leave them implicit whenever we draw a quiver diagram.

We let $\bar{Q}$ denote the resulting graded quiver after adjoining the opposite arrows and loops. ${ }^{5}$

We will soon incorporate potentials, which are linear combinations of cycles. These will allow us to consider quivers that contain loops (i.e., adjoints) and 2-cycles.

\section{Double arrows}

It is convenient to combine every $\varphi_{i j}^{(c)}$ with its corresponding $\varphi_{\mathrm{op}, j i}^{(m-c)}$ to form a double arrow, as illustrated in Fig. $1 .^{6}$ In this extended notation, the subscripts are the nodes connected by an arrow, and the superscript indicates its degree. We will refer to such a pair as a $(c, m-c)$ arrow. The number of different types of double arrows, i.e., the number of $(c, m-c)$ pairs with $0 \leq c \leq m / 2$, is

$$
n_{f}=\lfloor(m+1) / 2\rfloor \text {. }
$$

Double arrows of $(0, m)$ type exists for any $m$. Motivated by physics, we refer to such arrows as chiral fields. Furthermore, for even $m$, there is always a type of

\footnotetext{
${ }^{4}$ In other references, such as Ref. [14], the degree of an arrow is instead referred to as the color. Graded quivers are consequently also called colored quivers.

${ }^{5}$ In Ref. [15], Oppermann also includes the identity $\left(\varphi_{\mathrm{op}}\right)_{\mathrm{op}}=$ $(-1)^{|\varphi|(m-|\varphi|)+1} \varphi$ as part of the definition. We gloss over this technicality for the time being.

${ }^{6}$ This also applies to adjoint arrows, for which $i=j$.
} 


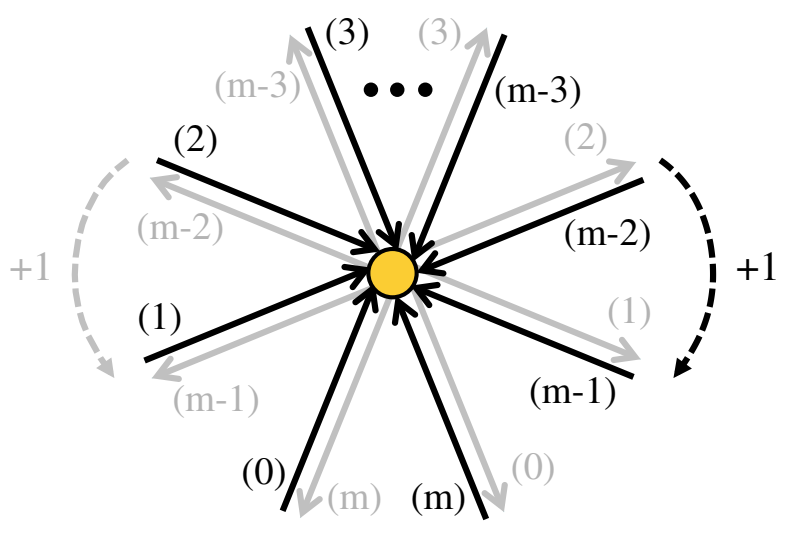

FIG. 2. Cyclic ordering of arrows connected to a node. The degree of incoming arrows increases clockwise.

$(m / 2, m / 2)$ arrows, in which both components have the same degree.

The (graded) signed adjacency matrix $q$ is skew symmetric, which in the context of graded quivers means that

$$
q_{i j}^{(c)}=q_{j i}^{(m-c)}
$$

\section{Remark 1}

We will not impose the monochromaticity condition of Ref. [14]; i.e., we will not require that if $q_{i j}^{(c)} \neq 0$ then $q_{i j}^{\left(c^{\prime}\right)}=0$ for $c \neq c^{\prime}$.

\section{Cyclic order}

Let us focus on a node in the quiver. Taking into consideration both the degrees of arrows and their incidence orientation with respect to the node, there are $(m+1)$ different possibilities. There is a natural cyclic order for arrows around the node, in which the degree of incoming arrows increases clockwise, as shown in Fig. 2. There might be multiple or no arrows of each type.

This order will become handy when discussing mutations and, as explained in Sec. X, also arises from mirror symmetry.

\section{Double arrows as quantum fields and their orientation}

Perhaps it is not surprising that a framework that allows multiple types of arrows can be useful for describing gauge theories in different dimensions. Such theories can contain different type of superfields, which, as we discuss below, are captured by the different types, i.e., degrees, of arrows in the quiver.

Given a double arrow, the distinction between $\varphi_{i j}^{(c)}$ and $\varphi_{\mathrm{op}, j i}^{(m-c)}$ is arbitrary. As we will explain in Sec. VIII, in cases with known physical interpretations as quantum field theories, every double arrow corresponds to a matter superfield. More generally, a double arrow should be regarded as a single entity. With this in mind, it is natural to associate an orientation to double arrows. Without loss of generality, let us assume that $0 \leq c \leq m / 2$. We will adopt the convention

$$
\left(\varphi_{i j}^{(c)}, \varphi_{\mathrm{op}, j i}^{(m-c)}\right) \rightarrow \begin{cases}\text { evenc: } & \Phi_{i j}^{(c)} \\ \text { oddc: } & \Phi_{j i}^{(c)},\end{cases}
$$

which is defined such that it coincides with the orientation of fields in quantum field theories. In other words, $\varphi_{i j}^{(c)}$ and $\varphi_{\mathrm{op}, j i}^{(m-c)}$ should be identified with physical fields or their conjugates as follows:

\begin{tabular}{c|c|c} 
& $\varphi_{i j}^{(c)}$ & $\varphi_{\mathrm{op}, j i}^{(m-c)}$ \\
\hline even c & $\Phi_{i j}^{(c)}$ & $\bar{\Phi}_{i j}^{(c)}$ \\
\hline odd c & $\bar{\Phi}_{j i}^{(c)}$ & $\Phi_{j i}^{(c)}$
\end{tabular}.

For brevity, in what follows, we will often use the terms "double arrow" and "field" interchangeably.

In the special case of even $m$, there is an ambiguity in identifying the field associated to an $(\mathrm{m} / 2, \mathrm{~m} / 2)$ double arrow. We can pick the corresponding field to either be $\Phi_{i j}^{(m / 2)}=\varphi_{i j}^{(c)}$ or $\Phi_{j i}^{(m / 2)}=\varphi_{\mathrm{op}, j i}^{(c)}$. This is possible because such fields are unoriented but, more importantly, are a manifestation of a $\Phi_{i j}^{(m / 2)} \leftrightarrow \bar{\Phi}_{i j}^{(m / 2)}$ symmetry of such theories. ${ }^{7}$ This issue will be revisited in the coming sections.

The orientation of fields becomes more significant in physics, in which it enters the determination of anomalies. The orientation in (2.4) is nicely consistent with the generalization of anomalies to arbitrary $m$ that we will introduce in Sec. VI.

Finally, in the cases with a gauge theory interpretation, we can identify the loops $\ell_{i}$ at every node with vector superfields. ${ }^{8}$ Since these loops are in one-to-one correspondence with nodes in the quiver and we will leave them implicit, we can simply say, as it is standard, that nodes correspond to vector multiplets. Notice that the $\ell_{i}$ 's are special in that graded quivers do not include their conjugates; they would be $\ell_{\mathrm{op}, i}$ 's. This is in nice agreement with physics, in which vector superfields satisfy a reality condition.

\footnotetext{
${ }^{7}$ Notice that we are not saying that $\Phi_{i j}^{(m / 2)}$ and $\bar{\Phi}_{i j}^{(m / 2)}$ are equal. This symmetry is a generalization to all even $m$ of the wellknown Fermi-conjugate Fermi symmetry of $2 \mathrm{D} \mathcal{N}=(0,2)$ gauge theories. This symmetry acts on each unoriented field independently.

${ }^{8}$ More precisely, we mean gauge supermultiplets. In particular, we refer to the corresponding superfield in zero dimensions as a gaugino superfield, since it has no vector component. For brevity, this distinction will be implicit throughout most of the paper.
} 


\section{Single arrow representation}

For simplicity, throughout the paper, we will often focus on a single arrow representative for every double arrow. A way of doing so is by simply picking any of the two arrows, let us call it $\varphi_{i j}^{(c)}$, keeping $\varphi_{\mathrm{op}, j i}^{(m-c)}$ implicit. In what follows, the choice of representative in a double arrow will be guided by practical purposes. Another natural way of picking a single-arrow representation is by using the physical orientation we introduced above. This will be the approach we will use when connecting to gauge theories in Sec. VIII.

\section{B. Ranks}

We complete the definition of a graded quiver by assigning an integer $N_{i}$ to every node. This ingredient is typically absent in the math literature. In physics, each node corresponds to an $U\left(\left|N_{i}\right|\right)$ gauge group, so we refer to these integers as ranks.

It is natural to restrict ourselves to positive ranks. Negative ranks can even be generated when starting from quivers with non-negative ranks and applying a sequence of mutations. In physics, the presence of negative ranks is typically an indication of SUSY breaking. It would be interesting to determine whether SUSY breaking has a mathematical counterpart.

For nontrivial ranks, arrows connecting nodes $i$ and $j$ become $\left|N_{i}\right| \times\left|N_{j}\right|$ matrices. ${ }^{9}$ The matrix structure of arrows will be implicit in our presentation.

\section{Potentials}

In this section, we extend the theory of graded quivers to include potentials. Our discussion is closely related to the one in Ref. [15].

The potential $W$ is a $\mathbb{C}$-linear combination of certain cycles in the path algebra $k \bar{Q}$, excluding the loops $\ell_{i}{ }^{10}$ More precisely, $W \in k \bar{Q} /[k \bar{Q}, k \bar{Q}]$, where we quotient by the supercommutator $[u, v]=u v-(-1)^{|u||v|} v u$. In other words, a cycle is an equivalence class of words made by closed paths of arrows up to sign as defined by

$$
\begin{aligned}
& \left(u_{1} u_{2} \cdots u_{k}\right)\left(v_{1} v_{2} \cdots v_{\ell}\right) \\
& \quad \sim(-1)^{\left(\left|u_{1}\right|+\left|u_{2}\right|+\cdots+\left|u_{k}\right|\right)\left(\left|v_{1}\right|+\left|v_{2}\right|+\cdots+\left|v_{\ell}\right|\right)}\left(v_{1} v_{2} \cdots v_{\ell}\right) \\
& \quad \times\left(u_{1} u_{2} \cdots u_{k}\right) .
\end{aligned}
$$

This is well defined since we assume the path is a cycle, i.e., closed with $t\left(v_{\ell}\right)=s\left(u_{1}\right)$.

\footnotetext{
${ }^{9}$ This is also the case when $i=j$.

${ }^{10}$ More generally, open paths terminating on frozen nodes can also be terms in the potential. In what follows, this additional possibility will be implicit whenever we refer to cycles. In physics, the cycle condition corresponds to gauge invariance.
}

In addition, cycles must have degree ${ }^{11}(m-1)$ in order to be allowed potential terms. The reasons for this will be explained momentarily in Sec. III. It is important to note that the potential $W$ does not necessarily contain all degree$(m-1)$ cycles. As an immediate important consequence of this restriction on the degree of the potential, there cannot be two arrows of the same type going in opposite directions along a cycle in the potential. For example, when considered with the same orientation, we cannot simultaneously have degree $c$ and $(m-c)$ for any $c$. Physically, no potential term can simultaneously contain a type of superfield and its conjugate. Additionally, arrows of degree $m$ cannot appear in potentials of such degrees.

As we explain in Sec. III, potentials give rise to relations in the path algebra. In physics, they encode nongauge interactions.

\section{Further constraints on the potential: Kontsevich bracket}

The Kontsevich bracket, sometimes also referred to as necklace bracket, between two functions $f$ and $g$ of the arrows in a quiver is defined as

$$
\{f, g\}=\sum_{\Phi \in \bar{Q}}\left(\frac{\partial f}{\partial \Phi} \frac{\partial g}{\partial \bar{\Phi}}-\frac{\partial f}{\partial \bar{\Phi}} \frac{\partial g}{\partial \Phi}\right) .
$$

It is a generalization of a Poisson bracket defined by a quiver [19-21]. When evaluating this bracket, it is necessary to take into account the commutation rules for arrows, which for a pair of them is given by $u v \sim(-1)^{|u||v|} v u$.

The potential is required to satisfy the condition that the Kontsevich bracket vanishes,

$$
\{W, W\}=0 .
$$

In Sec. III, we will explain that this condition is necessary for the differential on the Ginzburg algebra to square to zero. The condition (2.7) leads to nontrivial constraints on the potential. In Sec. VIII, we will explicitly consider the Kontsevich bracket for $m=1,2,3$.

\section{Mass terms and removable 2-cycles}

Quadratic terms in the potential are of particular significance. In physics, they correspond to mass terms. Since the potential must have degree $(m-1)$, it is straightforward to classify all possible mass terms. For this, it is convenient to define the upper wedge of the plane. It corresponds to the wedge containing the $(0, m),(1, m-1), \ldots,(m, 0)$ sequence of arrows, as shown in Fig. 3.

Every consecutive pair of fields in the upper wedge forming a closed path in the quiver can be combined into a

\footnotetext{
${ }^{11}$ The degree of a product of arrows is the sum of the degrees of the constituent arrows.
} 


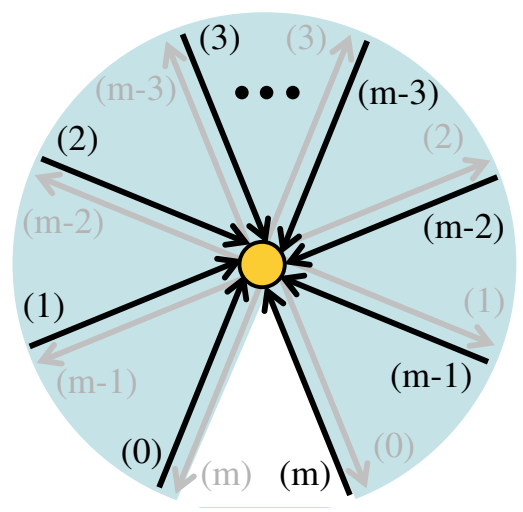

FIG. 3. The upper wedge.

quadratic term in the potential. We refer to such potential terms as mass terms.

We define a removable 2-cycle as a length-2 closed path in the quiver that, in addition, appears in a mass term in the potential. In this case, it is possible to integrate out the corresponding arrows, as we explain in Sec. IV B. ${ }^{12}$ In physics, a removable 2-cycle corresponds to a massive pair of fields. Note that chiral-chiral pairs can only form removable 2-cycles for $m=1$, for which $(0, m)$ and $(m, 0)$ fields are consecutive on the upper wedge.

\section{DIFFERENTIAL GRADED STRUCTURES}

We now introduce further structure that can be layered on top of a graded quiver with potential, combining together the treatments in Refs. [15,16].

\section{A. Differential operators}

For our final ingredient, we introduce a differential operator $d: k \bar{Q} \rightarrow k \bar{Q}$, which lowers the degree of a given term by 1 and then extends linearly. Furthermore, $d$ satisfies the graded Leibniz rule on products

$$
d(u v)=d(u) v+(-1)^{|u|} u d(v) .
$$

We define the differential $d$ on graded arrows as follows:

$$
d(\alpha)=0 \text { if } \alpha \text { has degree zero. }
$$

$d\left(\alpha_{\mathrm{op}}\right)=\partial_{\alpha} W$ if $\alpha$ has degree $\in\{0,1,2, \ldots, m-1\}$.

$$
d\left(\ell_{i}\right)=e_{i}\left(\sum_{\alpha \in Q_{1}}\left[\alpha, \alpha_{\mathrm{op}}\right]\right) e_{i} .
$$

Here, $e_{i}$ is the idempotent in the path algebra $k \bar{Q}$ at node $i$. The notation $\partial_{\alpha} W$ signifies the cyclic derivative, which is defined as $\partial_{\alpha}\left(v_{1} \cdots v_{k-1} \alpha\right)=v_{1} \cdots v_{k-1}$ and extended linearly. In the case in which $v=v_{1} v_{2} \cdots v_{k}$ is not written ending with $\alpha$, we use signed cyclic equivalence (2.5) to

\footnotetext{
${ }^{12}$ It is important to emphasize that 2-cycles cannot be removed if the corresponding term is not present in the potential.
}

move $\alpha$ to the rightmost position. ${ }^{13}$ As we will discuss later, the vanishings of all these differentials have important physical counterparts.

For later use, we also note that the right-hand side of (3.4) can be expressed as

$$
e_{i}\left(\sum_{\alpha \in Q_{1}}\left[\alpha, \alpha_{\mathrm{op}}\right]\right) e_{i}=\sum_{\alpha: i \rightarrow ? \in Q_{1}} \alpha \alpha_{\mathrm{op}}-\sum_{\beta: ? \rightarrow i \in Q_{1}} \beta_{\mathrm{op}} \beta
$$

for fixed $i \in Q_{0}$.

We now state two claims about differentials in our setting that will provide the backdrop for the correspondence between higher Ginzburg algebras and SUSY quantum field theories in various dimensions.

Claim 1: The fact that $W$ has degree $(m-1)$ implies that $d\left(\alpha_{\mathrm{op}}\right)=\partial_{\alpha} W$ indeed has degree one less than that of $\alpha_{\text {op }}$ (whenever $\alpha$ has degree ${ }^{14} \in\{0,1,2, \ldots, m-1\}$ ).

Proof: Suppose that $|\alpha|=c \in\{0,1,2, \ldots, m-1\}$ so that $\left|\alpha_{\mathrm{op}}\right|=(m-c) \in\{1,2, \ldots, m\}$. Then, in $\partial_{\alpha} W$, only the terms containing $\alpha$ at least once survive. Furthermore, each term resulting from $\partial_{\alpha} W$ is a term $v$ of $W$ with exactly one copy of $\alpha$ removed, which we abbreviate as $v \backslash \alpha$. Hence, if $W$ is homogenous of degree $(m-1)$, then $|v|=m-1$ and $|v \backslash \alpha|=(m-1)-|\alpha|=m-1-c$. We conclude that $d\left(\alpha_{\mathrm{op}}\right)=$ $\partial_{\alpha} W$ is homogeneous of degree $(m-c)-1$ as desired.

Claim 2: If the potential $W$ vanishes under the Kontsevich backet, i.e., $\{W, W\}=0$, then the differential defined above indeed squares to zero, i.e., $d^{2}=0 .{ }^{15}$

Proof: From Ref. [16], it follows that for $f \in k \bar{Q}$ we have $d f=\{W, f\}$. Hence, as in (10.6) of Ref. [16], we obtain

$$
d^{2} f=\{W,\{W, f\}\}=\frac{1}{2}\{\{W, W\}, f\}=0 .
$$

Furthermore, we have

$$
\begin{aligned}
d \ell_{i}= & d\left(\sum_{\alpha: i \rightarrow ? \in Q_{1}} \alpha \alpha_{\mathrm{op}}-\sum_{\beta: ? \rightarrow i \in Q_{1}} \beta_{\mathrm{op}} \beta\right) \\
= & \sum_{\alpha: i \rightarrow ? \in Q_{1}}\left(d(\alpha) \alpha_{\mathrm{op}}+(-1)^{|\alpha|} \alpha d\left(\alpha_{\mathrm{op}}\right)\right) \\
& -\sum_{\beta: ? \rightarrow i \in Q_{1}}\left(d\left(\beta_{\mathrm{op}}\right) \beta+(-1)^{\left|\beta_{\mathrm{op}}\right|} \beta_{\mathrm{op}} d(\beta)\right) \\
& +\sum_{\alpha: i \rightarrow ? \in Q_{1}}\left(\left(\partial_{\alpha_{\mathrm{op}}} W\right) \alpha_{\mathrm{op}}+(-1)^{|\alpha|} \alpha\left(\partial_{\alpha} W\right)\right) \\
& -\sum_{\beta: ? \rightarrow i \in Q_{1}}\left(\left(\partial_{\beta} W\right) \beta+(-1)^{\left|\beta_{\mathrm{op}}\right|} \beta_{\mathrm{op}}\left(\partial_{\beta_{\mathrm{op}}} W\right)\right) .
\end{aligned}
$$

\footnotetext{
${ }^{13}$ When a term $v=v_{1} v_{2} \cdots v_{k}$ of the potential $W$ contains $\alpha$ at multiple places, $\partial_{\alpha} v$ is the sum of the remainders after each such removal. More precisely, $\partial_{\alpha} v$ is defined as $\sum_{v=p \alpha q}(-1)^{|p \alpha||q|} q p$ in Ref. [15].

${ }^{14}$ In the special case in which $|\alpha|=m$, then $\left|\alpha_{\text {op }}\right|=0$, and we use (3.2) to compute $d\left(\alpha_{\text {op }}\right)$ instead.

${ }^{15}$ This property is in fact crucial because otherwise the map $d: k \bar{Q} \rightarrow k \bar{Q}$ defined above would not be a differential operator.
} 
Substituting in $\beta=\alpha_{\mathrm{op}}$, we note that these two sums are actually over the same arrows and cancel each other out. ${ }^{16}$ Hence, applying $d^{2}$ to the central element $\sum_{i \in Q_{0}} \ell_{i}$ yields zero.

\section{B. Higher Ginzburg algebras}

Given the three ingredients

(1) a graded quiver $\bar{Q}$ built from a quiver $Q$ with arrows $\alpha \in Q_{1},|\alpha| \in\{0,1,2, \ldots, m\}$, opposite arrows $\alpha_{\text {op }}$ for every such $\alpha$, and loops $\ell_{i}$ of degree $(m+1)$;

(2) a potential $W \in k \bar{Q} /[k \bar{Q}, k \bar{Q}]$ of degree $(m-1)$ satisfying $\{W, W\}=0$; and

(3) a differential operator $d: k \bar{Q}^{(j)} \rightarrow k \bar{Q}^{(j-1)}$ respecting the grading as above, ${ }^{17}$

we let the higher Ginzburg algebra $\Gamma_{m+2}(Q, W)$ denote the differential graded (dg) algebra given as the direct sum

$$
\Gamma_{m+2}(Q, W)=\oplus_{j \geq 0} k \bar{Q}^{(j)},
$$

where $k \bar{Q}^{(j)}$ denotes the space of paths of degree $j$ in the graded path algebra $k \bar{Q}$ and quotienting by the ideal of arrows.

The ordinary Ginzburg algebra from Refs. [22,23] corresponds to the $m=1$ case of the above. ${ }^{18}$

\section{Jacobian algebras and vacuum moduli spaces}

We now consider the following result of Ladkani, which in turn is a generalization of Ref. [23], Lemma 2.8. In particular, the result implies that it is sufficient to consider a quotient algebra formed by quotienting only by the relations arising from cyclic derivatives with respect to arrows of degree $(m-1)$.

Claim 3 (Ref. [24], Lemma 2.21): Let $(Q, W)$ be a quiver with potential in which $\bar{Q}$ is the associated graded quiver with degrees in $\{0,1,2, \ldots, m\}$. Then, the Jacobian algebra [with respect to the graded arrows of degree $(m-1)]$ is the zeroth cohomology ${ }^{19}$ of the complete Ginzburg dg algebra $\hat{\Gamma}_{m+2}(Q, W)$, i.e.,

$$
k \bar{Q} /\left(\left\{\partial_{\alpha} W: \alpha \in \bar{Q}_{1}^{(m-1)}\right\}\right)=H^{0}\left(\hat{\Gamma}_{m+2}(Q, W)\right) .
$$

\footnotetext{
${ }^{16}$ Technically, one uses footnote 5 to simplify the instances of $\left(\alpha_{\text {op }}\right)_{\text {op }}$ to $\pm \alpha$, but since the same sign change is applied twice, we still get zero.

${ }^{17}$ By convention, $k \bar{Q}^{(-1)}=0$, so using (3.2) we have $d: k \bar{Q}^{(j)} \rightarrow$ $k \bar{Q}_{18}^{(j-1)}$ even when $j=0$.

${ }^{18}$ Additionally, in Ref. [23], the authors focus on the completed Ginzburg dg algebra $\hat{\Gamma}(Q, W)$, i.e., the graded path algebra taking the limit of including paths of infinite length. This subtlety will not be needed in our work.

${ }^{19}$ In our language, this actually would be homology rather than cohomology, since we give arrows degrees that are positive rather than negative.
}

Proof: The zeroth homology is defined as

$$
\begin{aligned}
\text { Ker } & d: \hat{\Gamma}_{m+2}(Q, W)^{(0)} \\
& \rightarrow \hat{\Gamma}_{m+2}(Q, W)^{(-1)} / \operatorname{Im} d: \hat{\Gamma}_{m+2}(Q, W)^{(1)} \\
& \rightarrow \hat{\Gamma}_{m+2}(Q, W)^{(0)}
\end{aligned}
$$

where the superscripts indicate restricting to elements of the dg algebra $\hat{\Gamma}_{m+2}(Q, W)$ of certain degrees. Since we have no elements of degree $(-1)$, we get $\hat{\Gamma}_{m+2}(Q, W)^{(-1)}=0$, and hence the kernel is all of $\hat{\Gamma}_{m+2}(Q, W)^{(0)}$, i.e., the component of the graded path algebra $k \bar{Q}^{(0)}$ on arrows of degree 0 . We get the immediate equality $k \bar{Q}^{(0)}=k \bar{Q}$, the ordinary path algebra. Furthermore, the image consists of $\left\{d\left(\alpha_{\mathrm{op}}\right)\right\}$, where $\alpha_{\mathrm{op}}$ has degree 1 . Hence, the image consists of $\left\{\partial_{\alpha} W: \alpha \in \bar{Q}_{1}^{(m-1)}\right\}$, where the $(m-1)$ th component yields arrows in $\bar{Q}_{1}$ of degree $(m-1)$. In conclusion, we obtain the desired relations in the modified Jacobian algebra.

In the special case of $m=1$, the result in Ref. [23] relates the ordinary Ginzburg algebra to the ordinary Jacobian algebra, i.e., the quotient algebra $k Q /\left(\left\{\partial_{\alpha} W: \alpha \in Q_{1}\right\}\right)$.

The mathematical importance of the Jacobian algebra with respect to arrows of degree $(m-1)$, i.e., of next-tomaximal degree, has a physical counterpart. As we will see in Sec. VIII, it is all we need for computing the (classical) moduli spaces of the corresponding quantum field theories. The underlying reason is that, since the degree of the potential is $(m-1), H^{0}\left(\hat{\Gamma}_{m+2}(Q, W)\right)$ consists exclusively of chiral fields, which are the only fields containing scalar components. When determining the moduli space, we also demand the vanishing of (3.4). When it is expressed as in (3.5), it becomes clear that, when restricted to chiral fields, this condition corresponds to the vanishing of $D$-terms.

\section{Role of higher-degree arrows}

Since we focused solely on the Jacobian algebra, i.e., the zeroth homology, in the above, the reader might wonder what the roles of the higher-degree arrows and components of the (higher) Ginzburg algebra are. These higher-degree arrows and the differential operator are exactly defined so that all higher homologies of the (higher) Ginzburg algebra vanish.

In particular, in the $m=1$ case, notice that

$$
\begin{aligned}
H_{1}\left(\hat{\Gamma}_{m+2}(Q, W)\right) & =\operatorname{Ker} d: \hat{\Gamma}_{m+2}(Q, W)^{(1)} \\
& \rightarrow \hat{\Gamma}_{m+2}(Q, W)^{(0)} / \operatorname{Im} d: \hat{\Gamma}_{m+2}(Q, W)^{(2)} \\
& \rightarrow \hat{\Gamma}_{m+2}(Q, W)^{(1)}
\end{aligned}
$$

We note that $\hat{\Gamma}_{m+2}(Q, W)^{(2)}$ is generated by the loops $\ell_{i}$ and hence the image under $d$ is precisely generated by $e_{i}\left(\sum_{\alpha \in Q_{1}}\left[\alpha, \alpha_{\mathrm{op}}\right]\right) e_{i}$ as $i$ runs over possible nodes in $Q_{0}$. 
Meanwhile, the elements of $\hat{\Gamma}_{m+2}(Q, W)^{(1)}$ are the expressions in $k \bar{Q}$ of which the terms contain exactly one arrow $\alpha_{\text {op }} \in \bar{Q}_{1}^{(1)}$ and the rest in $\bar{Q}_{1}^{(0)}=Q_{1}$. The kernel of $d$ acting on this set is precisely the elements that become zero in $k \bar{Q}^{(0)}=k Q$ when $\alpha_{\text {op }}$ is replaced with $\partial_{\alpha} W \cdot{ }^{20}$ We will show that the kernel is in fact also generated by the elements of the form $e_{i}\left(\sum_{\alpha \in Q_{1}}\left[\alpha, \alpha_{\mathrm{op}}\right]\right) e_{i}$, which has image

$$
\sum_{\alpha: i \rightarrow ? \in Q_{1}} \alpha\left(\partial_{\alpha} W\right)-\sum_{\beta: ? \rightarrow i \in Q_{1}}\left(\partial_{\beta} W\right) \beta
$$

under $d$. This quantity indeed equals zero because the two summands express the sum over all terms in the potential $W$ incident to node $i$, in two different ways. In the positive summand, we sum over all terms of $W$ that contain an arrow $\alpha$ starting at $i$, while in the negative summand, we sum over all terms of $W$ that contain an arrow $\beta$ ending at $i$. However, since terms in $W$ are cycles, these two summands cancel each other out. For higher $m$, a related approach applies.

Construction 2.6 of Ref. [15] describes in more detail how one can start with a basic finite-dimensional algebra, e.g., one of the form $k Q /(R)$, where $k Q$ is a path algebra of a quiver $Q$ and $R$ is a minimal set of relations given by elements of the path algebra, and build a differential graded algebra. In particular, let the arrows of the original quiver $Q$ be of degree 0 and adjoin new arrows $\alpha_{r}$ of degree 1 for each of the relations $r \in R$, such that the differential map sends $\alpha_{r} \in k \bar{Q}^{(1)}$ to $d\left(\alpha_{r}\right)=r \in k \bar{Q}^{(0)}$. Consequently, $H_{0}\left(k \bar{Q}^{(1)}\right)=k \bar{Q}^{(0)} /(R)=\Lambda$. We then consider a generating set for $H_{1}\left(k \bar{Q}^{(1)}\right)$ and adjoin arrows of degree 2 for each element therein, defining the differential accordingly. Iterating this process, considering relations of relations, we get a differential graded algebra such that all higher homologies vanish.

Because all higher homologies vanish, the sequence of homologies of the graded (higher) Ginzburg algebra agrees identically with the sequence of homologies of the basic algebra $\Lambda$, treating this vacuously as a graded algebra (concentrated in degree 0 ). We conclude that the higher Ginzburg algebra $\Gamma_{m+2}(Q, W)$ and $\Lambda$ are quasi-isomorphic. As an application, when $\Lambda$ is the Jacobian algebra, it is quasi-isomorphic to $\Gamma_{m+2}(Q, W)$ while also agreeing to the zeroth homology of $\Gamma_{m+2}(Q, W)$.

However, because the higher Ginzburg algebra $\Gamma_{m+2}(Q, W)$ has the extra structure of a dg algebra, we observe that the higher Ginzburg algebra is $(m+2)$ Calabi-Yau. This means that $\Gamma_{m+2}(Q, W)$ is homology smooth and the shift functor $[m+2]$ is a Serre functor on the bounded derived category of finite-length $\Gamma_{m+2}(Q, W)$

\footnotetext{
${ }^{20}$ This calculation uses $d\left(u_{1} u_{2} \ldots u_{k} \alpha_{\text {op }}\right)=d\left(u_{1} u_{2} \ldots u_{k}\right) \alpha_{\text {op }}+$ $(-1)^{0} u_{1} u_{2} \ldots u_{k} d\left(\alpha_{\text {op }}\right)=u_{1} u_{2} \ldots u_{k} \partial_{\alpha} W$ if $\left|u_{1}\right|=\left|u_{2}\right|=\ldots=$ $\left|u_{k}\right|=|\alpha|=0$. In fact, for any such cyclic ordering, we have $d\left(u_{j+1} u_{j+2} \cdots u_{k} \alpha_{\text {op }} u_{1} u_{2} \ldots u_{j}\right)=u_{j+1} u_{j+2} \cdots u_{k} \partial_{\alpha} W u_{1} u_{2} \ldots u_{j}$.
}

modules. In particular, the composition of the suspension $[m+2]$ and duality yields a bimodule quasi-isomorphism (Ref. [22], Definition 3.2.3), i.e., a transformation that induces certain symmetries between spaces of homomorphisms under duality.

\section{ORDER- $(m+1)$ MUTATIONS}

In this section, we introduce mutations of graded quivers with potentials. Our treatment builds on the work in Refs. $[14,15]$. We explain how all the defining elements of a theory transform: the quiver in Sec. IVA, the potential in Sec. IV B, and the ranks in Sec. IV C. We postpone the explanation of how the differential transforms until Appendix B.

We will restrict to mutations on nodes without adjoint fields, i.e., without loops. Preliminary studies of this case have appeared in the mathematics literature $[15,25]$. However, we consider the understanding of such cases to be incomplete. It would be very interesting to revisit this problem.

\section{A. Mutation of the quiver}

Let us first explain how the graded quiver transforms under mutation:

(1) Flavors. - Let us consider a mutation on node $j$. In physics, the arrows connected to the mutated node are usually referred to as flavors. The flavors are

transformed as follows:
(1.a.) Replace every incoming arrow $i \stackrel{(c)}{\rightarrow} j$ with the arrow $i \stackrel{(c-1)}{\longrightarrow} j$.

(1.b.) Equivalently, replace every outgoing arrow $j \stackrel{(c)}{\rightarrow} k$ with an arrow $j \stackrel{(c+1)}{\rightarrow} k$.

Both of these values are taken modulo $(m+1)$.

This transformation has an elegant implementation in terms of the cyclic ordering of arrows introduced in Sec. II A. It simply becomes a rotation, as shown in Fig. 4, in which we have numbered the spectator nodes to emphasize that they remain fixed under the mutation.

(2) Composite arrows.-The second step in the transformation of the quiver involves the addition of composite arrows as follows. For every 2-path $i \stackrel{(0)}{\rightarrow} j \stackrel{(c)}{\rightarrow} k$ in $\bar{Q}$, where $c \neq m$, add a new arrow $i \underset{j}{j} k$.

In other words, we generate all possible composite arrows consisting of chiral fields, of degree 0 , coming into the mutated node and all other types of fields attached to it. In physics, such composite arrows are referred to as mesons.

\section{Anticomposition}

Equivalently, we can also understand the rule above as the composition of $\varphi_{i j}^{(0)}$ and $\varphi_{\mathrm{op}, k j}^{(m-c)}$, with $m-c \neq 0$, even though their orientations seem to be incompatible. The result is a meson that we can call $\varphi_{\mathrm{op}, i k}^{(m-c)}$, which is 

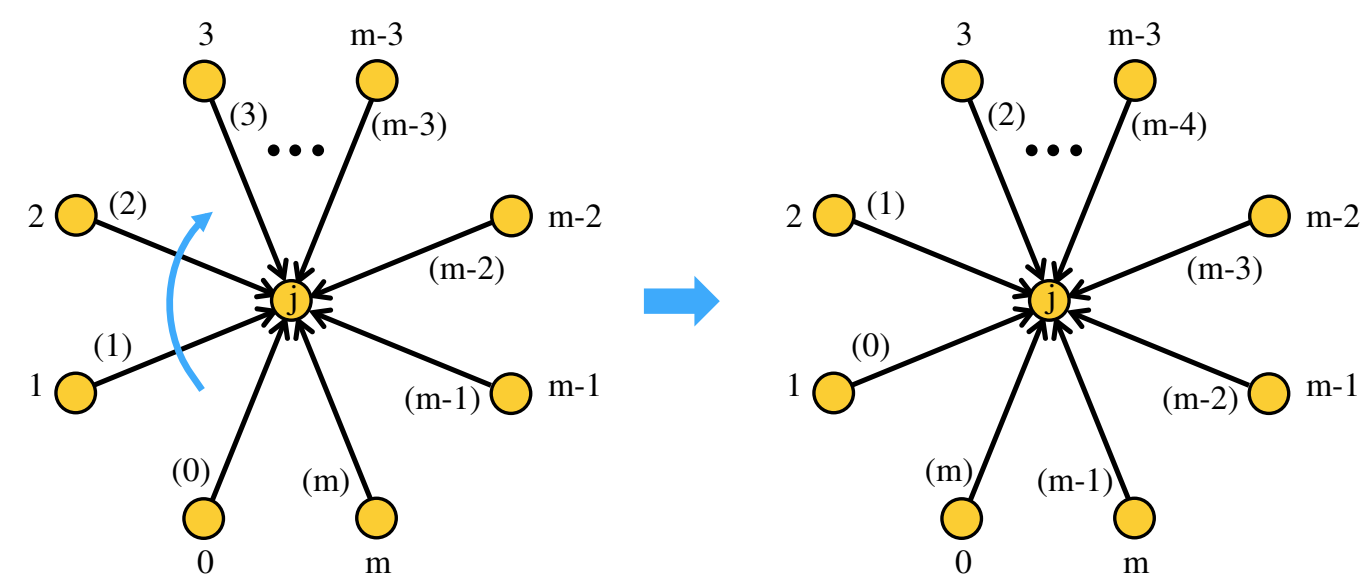

FIG. 4. The transformation of flavors upon a mutation on node $j$ can be implemented as a rotation of the degree of the arrows. The nodes remain fixed.

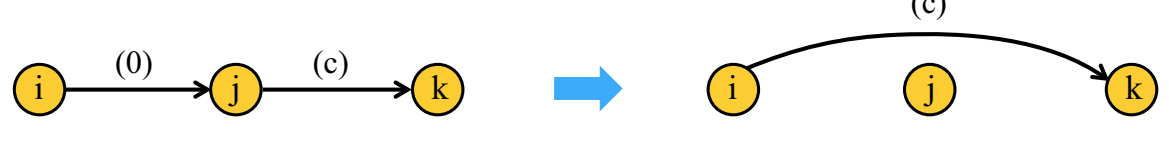

FIG. 5. Composite arrow, i.e., meson, generated by a mutation.
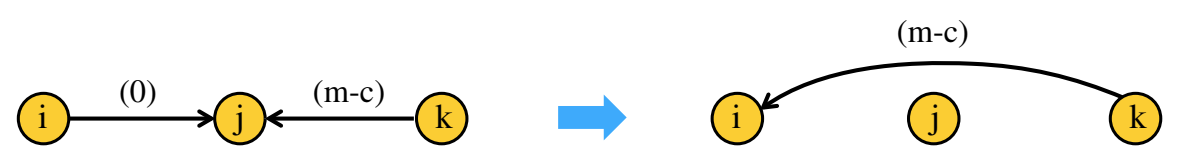

FIG. 6. Meson generated by anticomposition. Here, $m-c \neq 0$. This rule is equivalent to the one in Fig. 5 .

equivalent to a meson $\varphi_{k i}^{(c)}$. This phenomenon has been noted in both mathematics [15] and physics [3], and we refer to it as anticomposition. Anticomposition becomes important for $m \geq 3$. This alternative formulation of the composition rule is illustrated in Fig. 6.

Anticomposition becomes most shocking when focusing on the physical orientation of fields. In this case, a meson can correspond to the combination of a chiral field with another field of seemingly incompatible orientation, hence requiring the conjugation of the chiral field. This phenomenon was first noticed in Ref. [3] in the context of $0 \mathrm{D} \mathcal{N}=1$ quadrality. We will discuss it in further detail in Sec. IX.

It is worth noting we that we require $c \neq m$ in rule 2. In other words, the definition of composition forbids the anticomposition of two incoming chiral fields; i.e., we cannot generate a meson by composing an incoming chiral with the conjugate of another incoming chiral. ${ }^{21}$

\footnotetext{
${ }^{21}$ Our mutation rule for mesons coincides with some works in the mathematics literature such as Ref. [15], Sec. 6, but slightly differs from others, particularly Ref. [14]. In Appendix C, we elaborate on the relation between our mutation prescription and Ref. [14]. Contrary to ours, those rules do not follow from a proper consideration of the potential but have an equivalent effect when we restrict to higher cluster categories associated to graded quivers of Dynkin type, which is the problem of interest in Ref. [14].
}

\section{B. Mutation of the potential}

We now explain how the potential transforms under mutation, which can be summarized by a short set of rules. In Ref. [15], Oppermann provided an alternative, but equivalent, prescription for mutating the potential. Our approach is more combinatorial than his differential geometric treatment. The connection between the two is discussed in Appendix B.

(2.a.) Cubic dual flavors-meson couplings.-The first rule concerns new potential terms that are in one-to-one correspondence with mesons. For every 2-path, $i \stackrel{(0)}{\rightarrow} j \stackrel{(c)}{\rightarrow} k$ in $\bar{Q}$, with $c \neq m$, add the new arrow $i \stackrel{(c)}{\rightarrow} k$ in $\bar{Q}$ and the new cubic term $\varphi_{i k}^{(c)} \varphi_{j k}^{(c+1)} \varphi_{i j}^{(m)}=$ $\varphi_{i k}^{(c)} \varphi_{k j}^{(m-c-1)} \varphi_{j i}^{(0)}$ to $W$. Figure 7 shows the general form of these cycles.

Besides adding these new terms to the potential, the original terms can also be altered. Terms in the original potential that do not go through the mutated node remain unchanged. However, let us consider what happens to terms in the potential that contain the mutated node. There are two possibilities, depending on the degrees of the arrows that are connected to the mutated node in the corresponding cycle. These are handled by rule $2 . b$ in the first case and rules $2 . c$ and $2 . \mathrm{d}$ in the second. 


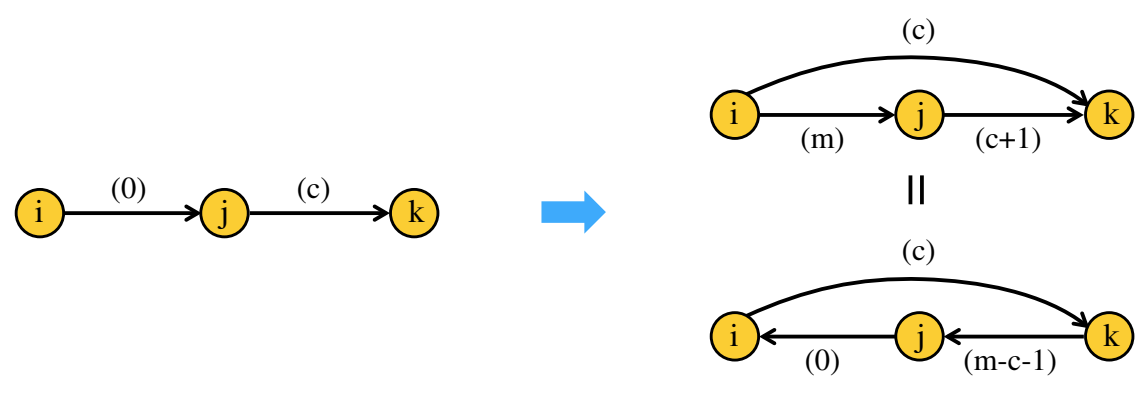

FIG. 7. New cubic terms coupling mesons to dual flavors.
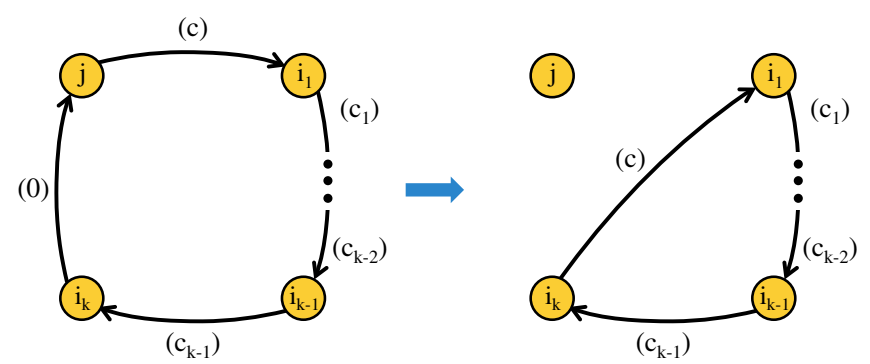

FIG. 8. Mutation of a potential term with a 2-path giving rise to a meson.

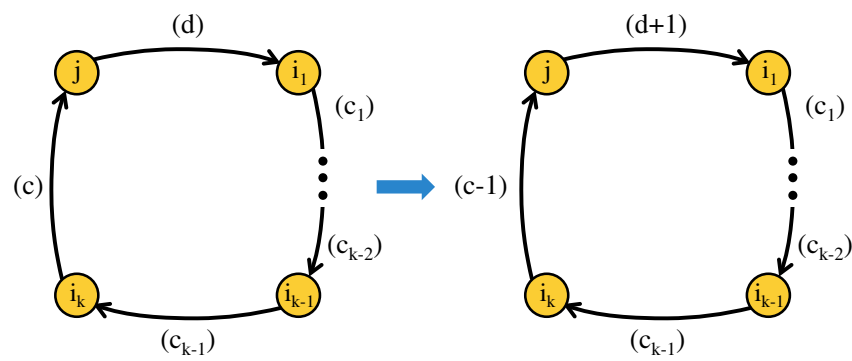

FIG. 9. Mutation of a potential term with a 2-path that goes through the mutated node but does not generate a meson.

(2.b.) Replace instances of $\varphi_{i j}^{(0)} \varphi_{j k}^{(c)}$ in $W$ with the meson $\varphi_{i k}^{(c)}$ obtained by composing the two arrows. Given the observation in Sec. II C, we know that $c \neq m$ (see Fig. 8). ${ }^{22}$

(2.c.) Replace instances of $\varphi_{i j}^{(c)} \varphi_{j k}^{(d)}$ in $W$, where $c \neq 0$ and $d$ is arbitrary (again, the case $d=m$ is already ruled out) with the product of dual flavors $\varphi_{i j}^{(c-1)} \varphi_{j k}^{(d+1)}$ (see Fig. 9).

(2.d.) Additionally, if there is an incoming chiral arrow $\varphi_{i_{0} j}^{(0)}$ at the mutated node, an additional term in $W$ is generated by duplicating this cycle but replacing instances of $\varphi_{i j}^{(c)} \varphi_{j k}^{(d)}$ with the product of mesons $\varphi_{i i_{0}}^{(c)} \varphi_{i_{0} k}^{(d)}$, which result from (anti)composing the

\footnotetext{
${ }^{22}$ The case $c=m$ is ruled out since this arrow is part of a potential term that has degree $(m-1)$.
}

original flavors $\varphi_{i j}^{(c)}$ and $\varphi_{j k}^{(d)}$, with $\varphi_{i_{0} j}^{(0)}$ (see Fig. 10). ${ }^{23}$ It is clear that whenever we apply $2 . \mathrm{d}$ we also apply 2.c.

Rules 2.c and 2.d are new features of graded quivers and are only relevant for $m \geq 2$. Interestingly, it is possible to distinguish the previous three rules by the number of mesons in the new potential terms. Rules 2.b, 2.c, and 2.d correspond to 1, 0, and $\geq 2$ mesons, respectively.

(3) Finally, we can apply reductions of mass terms, see Sec. II C 2, to get an equivalent graded quiver with potential. Massive fields are eliminated using the relations coming from the corresponding cyclic derivatives of the potential.

\section{Cycles that pass multiple times through the mutated node}

Additionally, in the preceding discussion, we have deliberately kept the nodes in potential cycles arbitrary. In particular, our rules also apply when a cycle passes through the mutated node multiple times. When this occurs, we simply apply the appropriate rules to all appearances of the mutated node in the cycle.

Rather than going into a lengthy analysis, it is probably better to illustrate this discussion with an explicit example. Figure 11 shows a potential cycle that passes twice through the mutated node $j$, which for clarity is shown in blue. Furthermore, $c, c^{\prime} \neq 0$, and there is an incoming chiral $\varphi_{i_{0} j}^{(0)}$. Both passings through node $j$ hence satisfy the conditions for rules 2.c and 2.d. Applying 2.c and 2.d in all possible ways, we obtain the four terms shown in Fig. 12. ${ }^{24}$

\section{Allowable potential terms and mutations}

As explained in Sec. II C, potential terms correspond to degree- $(m-1)$ cycles. Having explained how the potential

\footnotetext{
${ }^{23}$ Technically, to preserve $\left\{W^{\prime}, W^{\prime}\right\}=0$ once the potential $W$ is mutated to $W^{\prime}$, we in fact negate the coefficient in front of this additional term. However, we will not worry about the signs of potential terms in this exposition.

${ }^{24}$ Technically, the terms corresponding to the second and third cycles illustrated in this figure would have negative signs in front of them.
} 

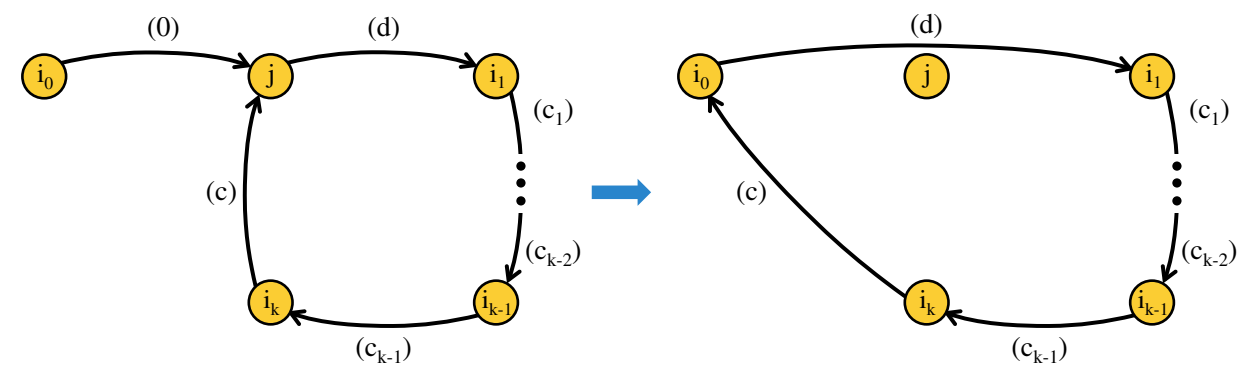

FIG. 10. Mutation of a potential term in the presence of an additional chiral field incoming into the mutated node.

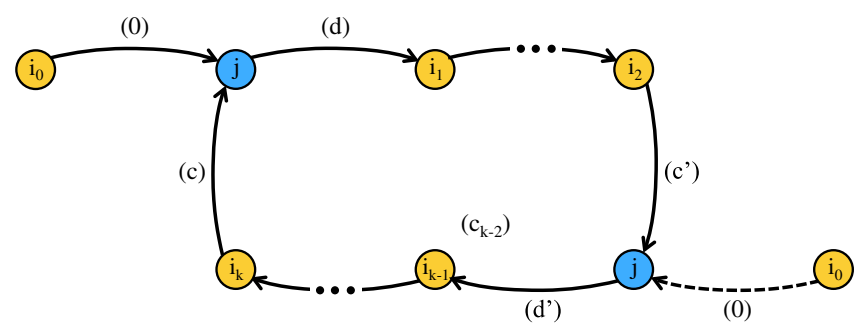

FIG. 11. A cycle going through the mutated node twice, such that the conditions for 2.d (and hence for 2.c) hold for both passings. The mutated node is shown in blue.

transforms under mutations, it is possible to show that any such cycle can be reached via a sequence of mutations from the basic configuration shown in Fig. 13. We prove this claim in Appendix A.

\section{Kontsevich bracket}

The transformation rules for the potential that we introduced imply that the $\{W, W\}=0$ condition is preserved by mutations. In Appendix B, we show that this is the case and present an alternative proof based on
Oppermann's ideas [15]. In the process, we will discuss connections between the two approaches.

\section{Mutation of the potential in combinatorial models}

It is worth noting that there exist various combinatorial models that can be interpreted as certain classes of graded quivers with potentials in which $m>1$. Going back to Ref. [14], the case of type- $A_{n} m$-graded quivers (called colored quivers therein) corresponds to $(m+2)$-angulations of an $(m n+m+2)$-gon. In toric cases when $m=2$, graded quivers and triality correspond to brane bricks and their transformations as studied in Refs. [4-6,9]. Both $(m+2)$ angulations and brane bricks can be modeled by potentials as defined in Sec. II C. The $(m+2)$-gons give rise to $\left(\begin{array}{c}m+2 \\ 3\end{array}\right)$ potential terms, each of which corresponds to a choice of three not necessarily consecutive edges on the boundary of the $(m+2)$-gon. For the brane brick models, potential terms correspond to edges of these three-dimensional cell complexes. Using these combinatorial models, one can describe how mutation affects potentials by comparing the potentials associated to the $(m+2)$-angulation (respectively, brane brick model) before or after mutation. The mutation of the potentials in these classes of combinatorial models coincides
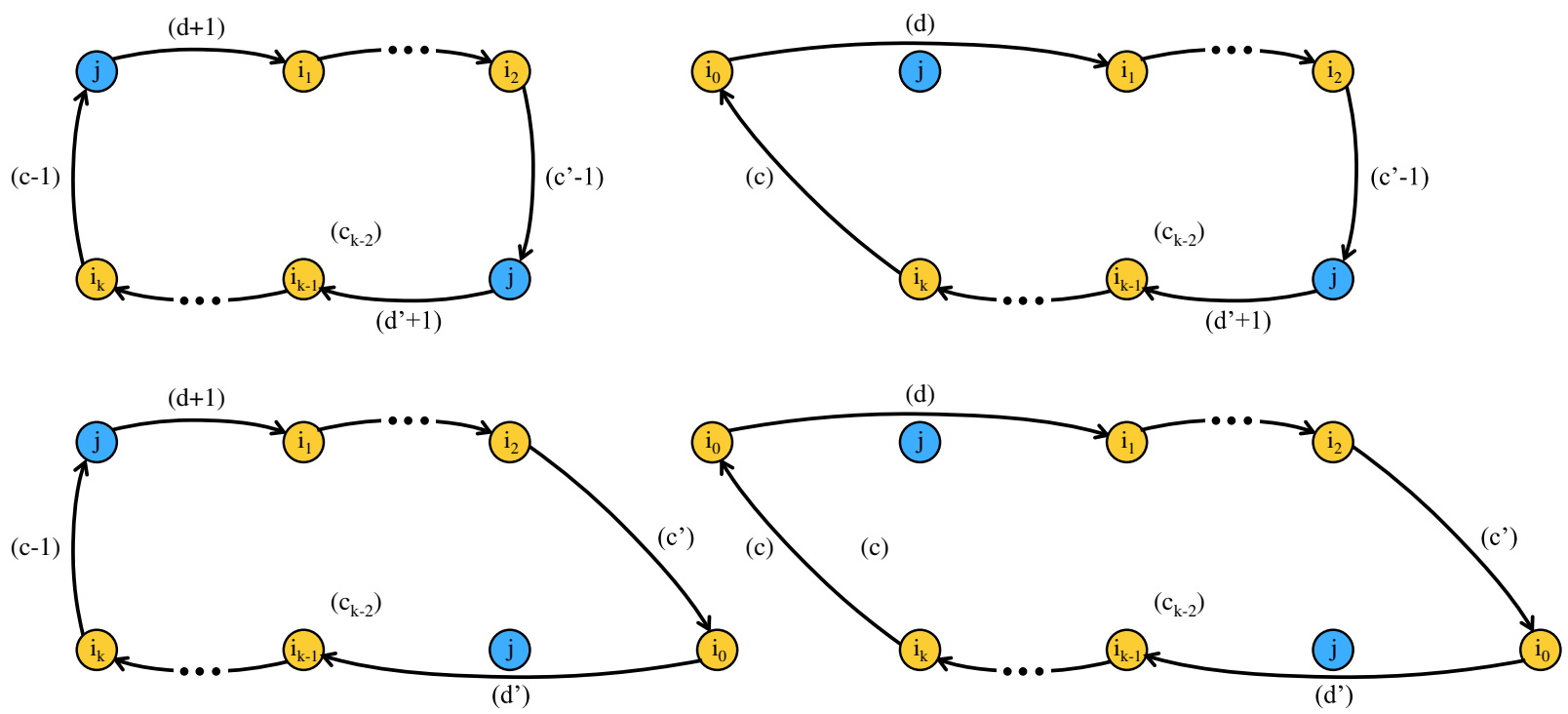

FIG. 12. The four terms generated by the cycle in Fig. 11 upon mutating on node $i_{0}$. 


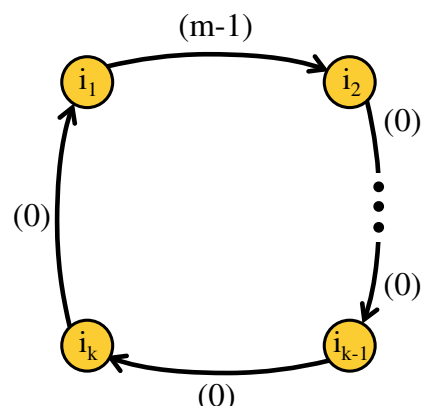

FIG. 13. Allowable potential terms can be connected by mutations to this basic configuration.

with our general rules, providing further motivation for their study.

\section{Mutation of the ranks: Fractional brane charges and $c$ vectors}

We postulate that the rank $N_{\star}$ of a mutated node transforms as

$$
N_{\star}^{\prime}=N_{0}-N_{\star},
$$

where $N_{0}$ indicates the total number of incoming chiral fields. More generally, we will indicate with $N_{c}$ the total number of incoming arrows of degree $c$. While we opt for keeping our notation as simple as possible, allowing several nodes for each degree and multiple arrows between nodes is straightforward.

Equation (4.1) coincides with the transformation of ranks for $m=1,2$, and 3, for which the mutations can be interpreted as quantum field theory dualities, as explained in Sec. IX. It is natural to assume, as we will do, that this mutation rule extends to arbitrary $m$. Below, we motivate this proposal by discussing fractional brane charges and $c$ vectors, suggesting a connection between these two classes of objects along the way. Further motivation coming from higher cluster categories is provided in Appendix C.

\section{Fractional brane charges}

In quivers with a brane realization, every node $i$ is associated to a fractional brane charge vector $Q_{i}$, the dimension of which is equal to $n$, the total number of gauge groups in the quiver. The number of arrows between a pair of nodes $i$ and $j$ is controlled by the intersection number $\left\langle Q_{i}, Q_{j}\right\rangle{ }^{25}$ We assume that objects with these properties exist for quivers without a D-brane realization.

Without loss of generality, we can focus on a local configuration of the quiver as shown in Fig. $14 .{ }^{26}$ In this case, the multiplicity of fields is absorbed in the $N_{c}$ 's. In

\footnotetext{
${ }^{25}$ Whether the intersection pairing is symmetric or antisymmetric and the details regarding the degree and orientation of arrows depend on $m$.

${ }^{26}$ This does not mean that there are no additional nodes in the quiver, which would determine the dimension $n$ of the $Q_{i}$ vectors.
}

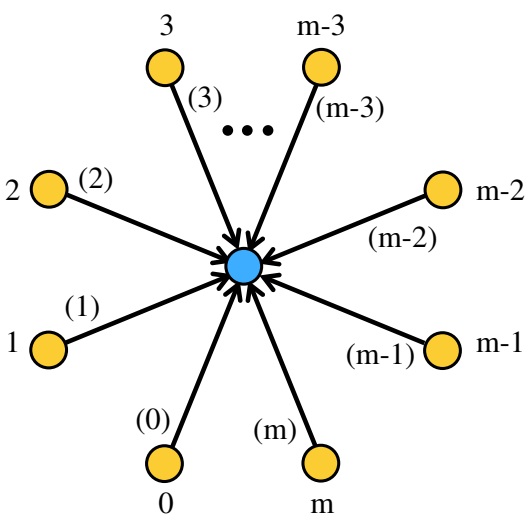

FIG. 14. Basic initial quiver. We consider consecutive mutations of the blue node.

particular, $\left\langle Q_{0}, Q_{\star}\right\rangle=1$. As will be discussed in Sec. X 2, mutation comes from a simple reorganization of the brane system, moving the branes associated to the mutated node over the ones that contribute incoming chirals to it. In this process, brane charges transform as

$$
\begin{aligned}
Q_{\star}^{\prime} & =-Q_{\star} \\
Q_{0}^{\prime} & =Q_{0}+\left\langle Q_{0}, Q_{\star}\right\rangle Q_{\star}=Q_{0}+Q_{\star} \\
Q_{1}^{\prime} & =Q_{1} \\
& \vdots \\
Q_{m}^{\prime} & =Q_{m},
\end{aligned}
$$

where we have naturally extended the known rule for $m \leq 3$ to arbitrary $m$. This rule leads to the appropriate transformation of the quiver. We refer the reader to Refs. [3,9,17] for discussions of the $m=1,2,3$ cases.

Focusing on the initial configuration in Fig. 14, the total initial brane charge is

$$
Q_{T}=N_{\star} Q_{\star}+\sum_{i=0}^{m} N_{i} Q_{i} .
$$

After mutation, the total brane charge becomes

$$
\begin{aligned}
Q_{T}^{\prime} & =N_{\star}^{\prime} Q_{\star}^{\prime}+\sum_{i=0}^{m} N_{i}^{\prime} Q_{i}^{\prime} \\
& =Q_{T}+\left[\left(N_{0}-N_{\star}\right)-N_{\star}^{\prime}\right] Q_{\star},
\end{aligned}
$$

where we have used that only $N_{\star}, Q_{\star}$, and $Q_{0}$ are modified. Conservation of the total brane charge $Q_{T}^{\prime}=Q_{T}$ requires that the second term vanishes, implying that the rank of the mutated node transforms as in (4.1).

\section{2. $c$ vectors: The $m=1$ case}

What are the mathematical counterparts of fractional branes charges? We now argue that they are (some generalization of) $c$ vectors. 
The following discussion is restricted to the $m=1$ case. In the original formulation of cluster algebras from Fomin and Zelevinsky [26], a seed for a cluster algebra is determined not only by an $n$-by- $n$ skew-symmetrizable matrix $B^{0}$ (equivalently a quiver on $n$ vertices) and by an initial cluster $\left\{x_{1}, x_{2}, \ldots, x_{n}\right\}$ but also by the data of a coefficient $2 n$-tuple $\left\{p_{1}^{ \pm}, p_{2}^{ \pm}, \ldots, p_{n}^{ \pm}\right\}$. The coefficients play a role in the binomial exchange relations

$$
x_{k} x_{k}^{\prime}=p_{k}^{+} \prod_{i=1}^{n} x_{i}^{\left[b_{k i}^{0}\right]_{+}}+p_{k}^{-} \prod_{i=1}^{n} x_{i}^{\left[-b_{k i}^{0}\right]_{+}}
$$

where $[\alpha]_{+}=\max (\alpha, 0)$. In their follow-up work [27], Fomin and Zelevinsky reexpress such seeds ${ }^{27}$ using coefficient $n$-tuples $\left\{y_{1}, y_{2}, \ldots, y_{n}\right\}$, extend the matrix $B^{0}$ to a $2 n$-by- $n$ matrix starting with appending the $n$-by- $n$ identity matrix underneath, and use the binomial exchange relations

$$
x_{k} x_{k}^{\prime}=\prod_{i=1}^{n} x_{i}^{\left[b_{k i}^{0}\right]_{+}} \prod_{j=n+1}^{2 n} y_{j-n}^{\left[b_{k i}^{0}\right]_{+}}+\prod_{i=1}^{n} x_{i}^{\left[-b_{k i}^{0}\right]_{+}} \prod_{j=n+1}^{2 n} y_{j-n}^{\left[-b_{k i}^{0}\right]_{+}}
$$

instead.

As we mutate the seed of a cluster algebra, the extended skew-symmetrizable matrix $\left[\begin{array}{c}B^{0} \\ I\end{array}\right]$ mutates according to the same rules as quiver mutation, and after a generic sequence of mutations, $\left[\begin{array}{c}B^{0} \\ I\end{array}\right]$ becomes $\left[\begin{array}{c}B \\ C\end{array}\right]$, where $C=\left[c_{i j}\right]_{i=1, j=1}^{n, n}$ is an invertible $n$-by- $n$ integer matrix. We refer to the columns of this $C$ matrix as $c$ vectors and denote them as $\mathbf{c}_{j}$ as $j=1, \ldots, n$.

Based on the quiver mutation rules, it follows that the $c$ vectors satisfy the following recurrence, e.g., see (2.9) of Ref. [28]:

$$
c_{i j}^{\prime}= \begin{cases}-c_{i j} & \text { if } j=k \\ c_{i j}+c_{i k}\left[b_{k j}\right]_{+}+\left[-c_{i k}\right]_{+} b_{k j} & \text { if } j \neq k .\end{cases}
$$

This recurrence is also a tropicalization of the recurrence for coefficient tuples made up of $\mathbf{y}_{j}$ 's, letting the $c$ vector $\mathbf{c}_{j}=\left[c_{1 j} c_{2 j} \cdots c_{n j}\right]^{T}$ denote the exponent vector of $\mathbf{y}_{j}$ in terms of the initial coefficients $\left\{u_{1}, u_{2}, \ldots, u_{n}\right\}$ (i.e., $\left.\mathbf{y}_{j}=\prod_{i=1}^{n} u_{i}^{c_{i j}}\right)$. The coefficients, i.e., detropicalized $c$ vectors, also correspond to $\mathcal{X}$ coordinates of Fock and Goncharov [29,30].

We are now ready to investigate whether $c$ vectors are related to fractional brane charges. A quick comparison of (4.7) and (4.2) reveals various similarities. To facilitate the comparison, it is convenient to translate (4.7) to the language we used to discuss fractional branes. Given $c_{i j}$,

\footnotetext{
${ }^{27}$ Here, we focus on the case of cluster algebras of geometric type with principal coefficients.
}

the $j$ index indicates a node, and $i$ runs over the components of the $c$ vector. In other words, we can identify $c_{i j}=Q_{j, i}$. Recall that we are working with $m=1$, so we only have $Q_{\star}$ for the mutated node and $Q_{0}$ and $Q_{1}$ for the incoming and outgoing chirals, respectively. If we work in the convention in which $b_{\star 0} \geq 0$, it implies that $b_{\star 1} \leq 0$ and hence $\left(b_{\star 1}\right)_{+}=0$. Furthermore, in supersymmetric configurations, $\left(-Q_{\star, i}\right)_{+}=0 .{ }^{28}$ With all this, Eq. (4.7) becomes

$$
\begin{aligned}
& Q_{\star}^{\prime}=-Q_{\star} \\
& Q_{0}^{\prime}=Q_{0}+b_{\star 0} Q_{\star} \\
& Q_{1}^{\prime}=Q_{1}
\end{aligned}
$$

in agreement with (4.2).

We conclude that for $m=1 c$ vectors can be identified with fractional brane charges. Our discussion of fractional brane charges suggest that the formulas for the ordinary quiver case, i.e., $m=1$, naturally lift to the case of $m \geq 1$. Thus, in future work, we wish to develop a theory of $c$ vectors for graded quivers with arbitrary $m$.

\section{PERIODICITY OF THE MUTATIONS}

We now show that the mutation introduced in previous sections is indeed an order- $(m+1)$ transformation, namely, that after $(m+1)$ consecutive mutations acting on the same node of a quiver we obtain the original theory.

It is sufficient to focus on the basic configuration shown in Fig. 14 as a starting point. In this figure, some of the ranks of the flavor nodes might be zero; i.e., flavors of the corresponding degrees might be absent. Our discussion extends to general initial configurations. We consider consecutive mutations of the blue node.

The transformation of flavors is simply given by a rotation, as shown in Fig. 4. It is thus clear that flavors return to the original configuration after $(m+1)$ mutations.

Initially, there are no mesons stretching between external nodes in Fig. 14. Since mesons are created at every mutation, it is important to verify that all of them disappear by the end of the mutation sequence. Let us first focus on the pair of nodes 0 and $c$, initially corresponding to flavors of degrees $(0)$ and $(c)$, both with nonvanishing ranks. If any of the ranks are zero, the corresponding flavors will be absent, and no mesons between these two nodes will ever be generated.

Figure 15 shows a sequence of $c+1$ mutations acting on the blue node. After the first mutation, a meson of degree $(m-c)$ is created between the two nodes. This remains the only meson between nodes 0 and $c$ until the $(c+1)$ th mutation, after which a meson of degree $(c-1)$ in the opposite direction is generated. The sum of the degrees of

\footnotetext{
${ }^{28}$ It is natural to conjecture that having $\left[-Q_{\star, i}\right]_{+} \neq 0$ corresponds to the inclusion of antibranes. It would be interesting to explore this idea in further detail.
} 

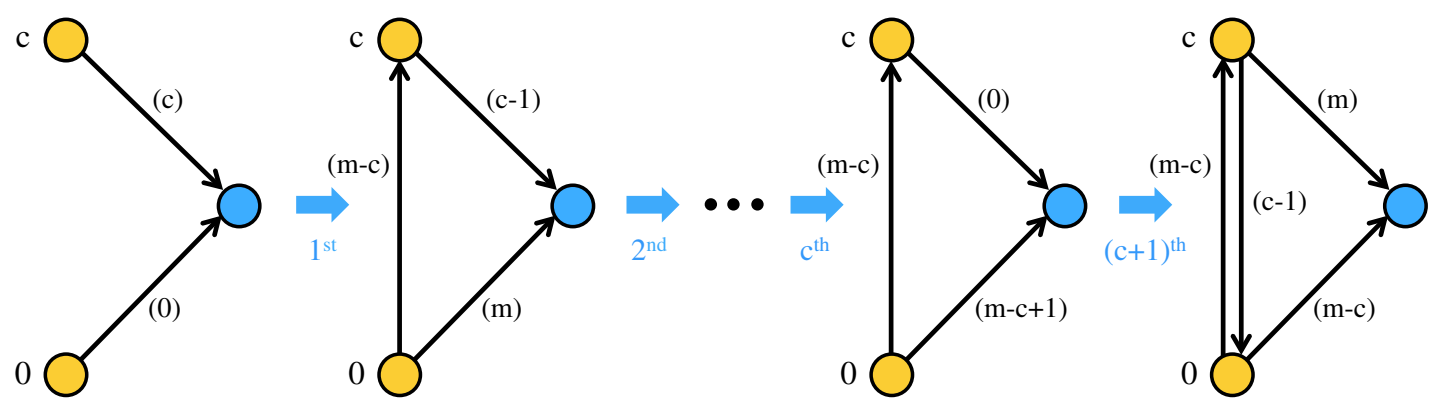

FIG. 15. Evolution of mesons connecting nodes 0 and $c$ under $c+1$ consecutive mutations of the blue node.

both mesons is $(m-1)$. In fact, they form a mass term in the potential and can be integrated out. We conclude that after $(c+1)$ mutations the mesons connecting nodes 0 and $c$ disappear. Given the transformation of flavors, only after $(m+2)$ mutations will we again generate a meson between nodes 0 and $c$.

Let us now consider a pair of arbitrary nodes $i$ and $j$, initially connected to flavors of degrees $c_{i}<c_{j}$. As shown in Fig. 16, after $c_{i}$ mutations, we reach the starting configuration of Fig. 15, with $c=c_{j}-c_{i}$. The analysis in the previous paragraph applies after this point. The meson generated at the $c_{i}$ th mutation, is removed after the $\left(c_{i}+1\right)$ th mutation by forming a massive pair with a meson going in the opposite direction. Since for any pair of nodes $c_{j}-c_{i} \leq m$, we conclude that after $(m+1)$ mutations we return to a configuration without mesons between external nodes.

The mutation rules in Sec. IV preserve the global symmetries of the theory. This in particular requires/implies that if the final quiver is identical to the initial one, as we have just shown, then the final potential also coincides with the original one.

We complete the proof of periodicity in the next section, in which we explain how, under certain conditions that generalize the cancellation of anomalies, the rank of the mutated node also returns to its original value after $(m+1)$ mutations.

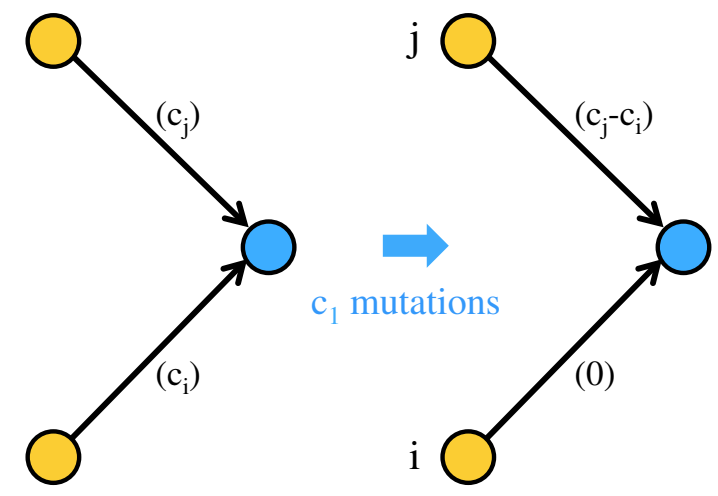

FIG. 16. Starting from a general pair of nodes, we reach the initial configuration in Fig. 15 after $c_{i}$ mutations.

\section{GENERALIZED ANOMALY CANCELLATION}

At present, physical interpretations of graded quivers as quantum field theories are only known for $m=1,2$, and 3 . This correspondence will be explained in Sec. VIII. Since anomalies play a central role in quantum field theories, it is reasonable to expect that they can be generalized to arbitrary $m$ and that they will remain important in this broader context. For example, in the case of $m=1$, cancellation of gauge anomalies requires at every node that the weighted number of incoming arrows equals the weighted number of outgoing arrows, with the weighting given by the ranks of the gauge groups.

From the discussion in Sec. IV C, in order for the rank of a node to return to the original value after $(m+1)$ consecutive mutations on it, we must have

$$
N_{\star}=\sum_{c=0}^{m}(-1)^{m-c} N_{c}+(-1)^{m+1} N_{\star} .
$$

Here, $N_{\star}$ is the rank of the node $\star$, the node to be mutated, and $N_{c}$ equals the number of arrows of degree $c$ incoming into that node as part of a double arrow. For $m=1,2$, and 3, this agrees with the cancellation of the gauge anomaly, as will be discussed in Sec. VIII. ${ }^{29}$ We will thus promote (6.1) to a generalized anomaly cancellation condition for arbitrary $m$. It is in fact quite remarkable that anomaly cancellation emerges from the periodicity condition of theories under mutations.

Equation (6.1) becomes (locally at every node)

$$
\begin{aligned}
\text { odd } \mathrm{m}: 0 & =N_{m}-N_{m-1}+\cdots-N_{1}+N_{0} \\
\text { even } \mathrm{m}: 2 N_{\star} & =N_{m}-N_{m-1}+\cdots-N_{1}+N_{0} .
\end{aligned}
$$

The rank of the gauge group $N_{\star}$ only enters anomaly cancellation for even $m$.

The preceding discussion of generalized anomalies is based on periodicity under $(m+1)$ consecutive mutations

\footnotetext{
${ }^{29}$ Here, we focus on the anomalies associated to the $S U\left(N_{i}\right)$ factors in the $U\left(N_{i}\right)=S U\left(N_{i}\right) \times U(1)$ gauge groups. We will not consider anomalies involving the $U(1)$ factors, which in D-brane constructions can be canceled by a stringy mechanism.
} 
of the same node. Since we have not defined mutation in the presence of adjoint fields, our argument does not apply to nodes containing such fields. However, for $m \leq 3$, anomalies can instead be computed by calculating the appropriate loop diagrams. It is then possible to include the contribution of matter in arbitrary representations of the gauge group, which are controlled by certain group theoretic factors. It is reasonable to expect that by directly generalizing such expressions we can incorporate the contributions of other representations, including adjoints, to generalized anomalies.

The convention of (2.4) ensures that the signs of the terms in (6.2) coincide with the ones for the corresponding physical fields.

To conclude this section, let us mention that frozen nodes can be anomalous. In physics, they correspond to global symmetry groups. The invariance of their anomalies under mutations of other nodes is called 't Hooft anomaly matching and constitutes a powerful constraint on dualities. More generally, mathematically, it is still interesting to consider theories with anomalous unfrozen nodes.

\section{MAP TO PHYSICS}

We are now ready to explain the connection between graded quivers with potentials and physics. Quivers with a maximum degree $m$ correspond to $(6-2 m)$-dimensional gauge theories with $2^{3-m}$ supercharges. More precisely, $m=1,2$, and 3 correspond to $4 \mathrm{D} \mathcal{N}=1,2 \mathrm{D} \mathcal{N}=(0,2)$, and $0 \mathrm{D} \mathcal{N}=1$ gauge theories, respectively. ${ }^{30}$ Such theories are called minimally supersymmetric. This correspondence will be explained in detail in Sec. VIII. Only theories with $m \leq 3$ have a standard gauge theory interpretation, since $m>3$ would naively correspond to theories in a negative number of dimensions. Interestingly, for any $m$, the graded quivers admit a physical realization in terms of the category of branes in the topological B-model on CY $(m+2)$-folds. This correspondence will be investigated in detail in a forthcoming paper [18].

It would be extremely interesting to determine whether there are physical systems described by graded quivers with $m>3$ or to detect a mathematical obstruction or qualitatively distinctive feature that first appears at $m=4$.

In physics, a quiver diagram summarizes the gauge symmetry and matter content of a quantum field theory. Nodes correspond to gauge groups, i.e., to vector superfields, and arrows indicate matter fields. As the dimension in which the field theory lives decreases, there are more types of matter superfields. This fact is nicely captured by the increasing number of possible degrees in the quiver as $m$ grows. The quiver diagram does not fully specify a minimally supersymmetric theory. To do so, additional information regarding interactions between matter fields

\footnotetext{
${ }^{30}$ In the coming section, we explain how many of the ideas presented here extend to the case of $m=0$.
}

TABLE I. D-brane configurations engineering quantum field theories in various dimensions.

\begin{tabular}{lccc}
\hline \hline$m$ & QFT & Original geometry & Mirror \\
\hline 1 & 4D $\mathcal{N}=1$ & IIB D3 probing CY & IIA D6 on 3-cycles \\
2 & 2D $\mathcal{N}=(0,2)$ & IIB D1 probing CY & IIB D5 on 4-cycles \\
3 & OD $\mathcal{N}=1$ & IIB D(-1) probing CY & IIA ED4 on 5-cycles \\
\hline \hline
\end{tabular}

needs to be provided. Such interactions are encoded in the potential.

A large class of these $(6-2 m)$-dimensional theories can be realized in type-IIB string theory on the world volume of $\mathrm{D}(5-2 m)$-branes probing $\mathrm{CY}(m+2)$-folds (see Refs. [3,4,31-34] and references therein). The probed CY manifolds emerge from the gauge theories as their classical moduli spaces. In this way, string theory provides a direct connection between these quivers and CY geometries, in nice parallel with the relationship based on (higher) Ginzburg algebras. Table I summarizes these setups and their mirror configurations. The use of mirror symmetry for understanding these theories is discussed in Sec. X.

When the CY $(m+2)$-folds are toric, a beautiful description of these theories in terms of objects that generalize dimer models exists. In this case, T-duality connects the $\mathrm{D}(5-2 m)$-branes probing $\mathrm{CY}(m+2)$-folds to new configurations of branes living on tori $\mathbb{T}^{m+1}$. For $m=1,2$, and 3 , these configurations are brane tilings [35,36], brane brick models [5,6,9], and brane hyperbrick models [3]. These constructions significantly streamline the connection between CY geometry and graded quivers in both directions. We envision profound connections between these combinatorial objects and the ideas presented in this paper. We postpone the exploration of this special toric case to future work.

\section{GAUGE THEORIES FOR $m=0,1,2,3$}

In this section, we discuss how the general framework of graded quivers with potentials with $m=0,1,2,3$ captures and unifies the physics of supersymmetric gauge theories in $d=6,4,2,0$, respectively. Moreover, our framework provides a systematic prescription for the transformation of the potentials under duality, which was not yet known for $d=2,0$. We include references with in-depth presentations of such quantum field theories. We start from $m=1$ and comment on $m=0$ toward the end.

$$
\text { A. } m=1: 4 \mathrm{D} \mathcal{N}=1
$$

Here, we explain how $m=1$ quivers correspond to 4D $\mathcal{N}=1$ gauge theories. There is a vast amount of literature on these theories; see, e.g., Refs. [37,38].

\section{Superfields}

Let us first discuss how the different elements in the quiver map to superfields. Nodes correspond to vector 


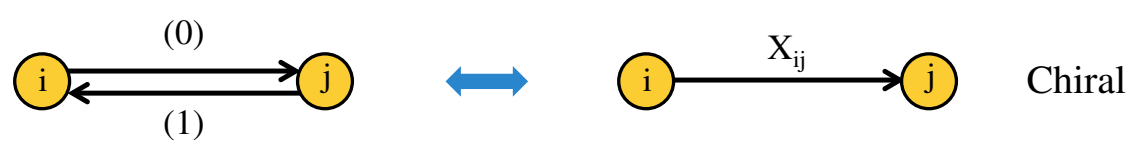

FIG. 17. $(0,1)$ arrows corresponds to $4 \mathrm{D} \mathcal{N}=1$ chiral fields.

multiplets. As explained earlier, it is sufficient to focus on arrows with degrees $0 \leq c \leq m / 2$, which can also be completed into $(c, m-c)$ double arrows. This means that in this case there is a single type of arrow, i.e., of matter superfield, which corresponds to $c=0$, i.e., to a $(0,1)$ double arrow. We identify such arrows with chiral superfields, as shown in Fig. 17. Here and in what follows, we determine the orientation of physical fields using the convention in (2.4). To follow standard 4D $\mathcal{N}=1$ notation, we call $X_{i j} \equiv \varphi_{i j}^{(0)}$. We conclude that $m=1$ quivers precisely match the most general field content of $4 \mathrm{D} \mathcal{N}=$ 1 gauge theories.

\section{Anomalies}

For every node, the generalized anomaly cancellation (6.2) takes the form

$$
0=N_{\chi_{\text {in }}}-N_{\chi_{\text {out }}},
$$

where $N_{\chi_{\text {in }}}$ and $N_{\chi_{\text {out }}}$ are the number of incoming and outgoing chiral fields, respectively. This is precisely the condition for the cancellation of the $S U\left(N_{\star}\right)^{3}$ gauge anomaly. The relative sign reflects the opposite contribution of fields transforming in the fundamental and antifundamental representations of $S U\left(N_{\star}\right)$.

\section{Potential}

Following the definition in Sec. II C, the degree of the potential for $m=1$ must be equal to 0 . Then, terms in the potential correspond to oriented cycles of chiral fields, as shown in Fig. 18. Different terms in the potential might involve different numbers of fields. In physics, we refer to the $m=1$ potential as the superpotential. An important property of the superpotential is that it is a holomorphic function of the chiral fields; i.e., it does not involve conjugate fields $\bar{X}_{i j}$.

The moduli space of these theories is determined by imposing vanishing $D$ and $F$-terms. The $F$-terms are the cyclic derivatives of the superpotential with respect to chiral fields. This agrees with (3.9).

For clarity, the figures in this section show potential terms containing a large number of fields.

\section{Kontsevich bracket}

Since the superpotential is holomorphic, the Kontsevich bracket vanishes automatically. This implies that, as is well known from physics, there is no additional constraint on the superpotential.

$$
\text { B. } m=2: 2 \mathrm{D} \mathcal{N}=(0,2)
$$

We now consider $m=2$ quivers, which correspond to $2 \mathrm{D} \mathcal{N}=(0,2)$ gauge theories. Thorough introductions to these theories can be found in Refs. [2,4,39,40].

\section{Superfields}

Once again, every node corresponds to a vector superfield. There are two types of arrows, associated to $c=0,1$. The resulting $(0,2)$ and $(1,1)$ double arrows correspond to chiral and Fermi superfields, respectively. Following standard notation, we will refer to chiral fields as $X_{i j}$ and Fermi fields as $\Lambda_{i j}$. Fermi fields are the first examples of $(m / 2$, $m / 2$ ) unoriented fields that we encounter in quantum field theories. Specifically, since $\varphi_{i j}^{(1)}$ and $\varphi_{\mathrm{op}, j i}^{(1)}$ have the same degree, we can identify the pair with either $\Lambda_{i j}$ or $\Lambda_{j i}$. The symmetry under the exchange of $\Lambda_{i j} \leftrightarrow \bar{\Lambda}_{i j}$ for any Fermi field is an important property of $2 \mathrm{D} \mathcal{N}=(0,2)$ gauge theories. Below, we will discuss this symmetry on the potential.

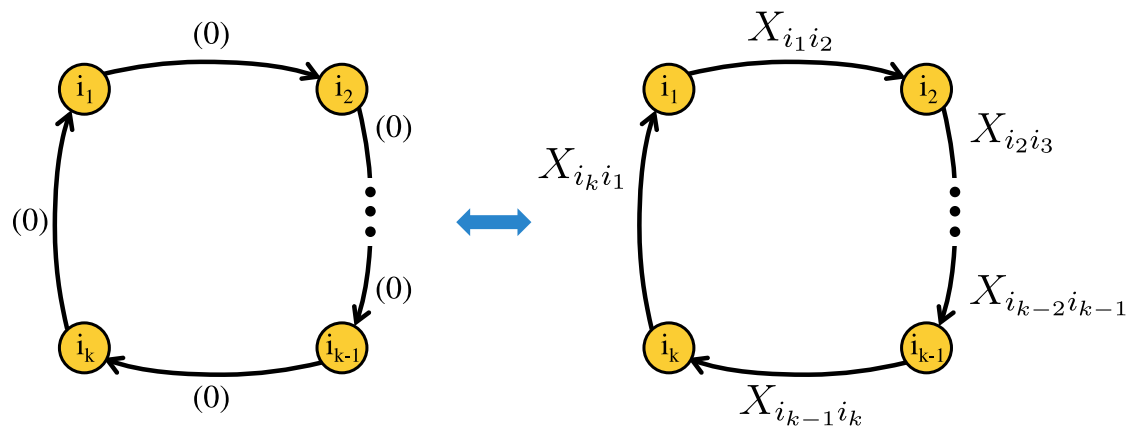

FIG. 18. The $m=1$ potential corresponds to the $4 \mathrm{D} \mathcal{N}=1$ superpotential. 


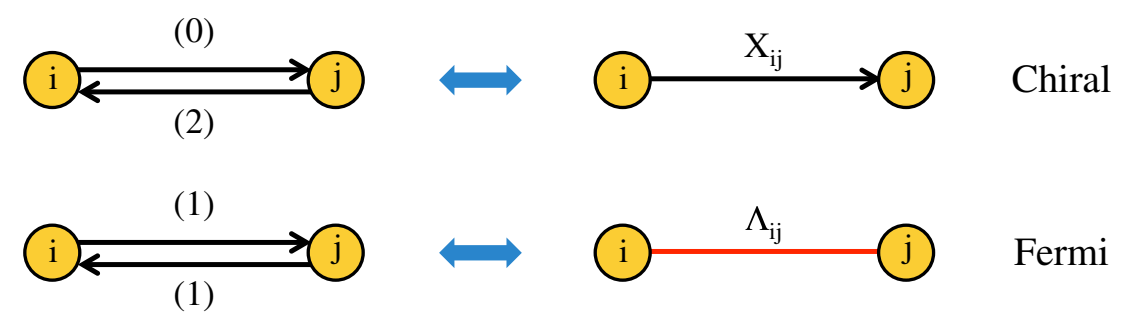

FIG. 19. $(0,2)$ and $(1,1)$ arrows correspond to $2 \mathrm{D} \mathcal{N}=(0,2)$ chiral and Fermi fields, respectively.

Figure 19 shows the map between arrows in an $m=2$ graded quiver and matter fields in a $2 \mathrm{D} \mathcal{N}=(0,2)$ theory. Given the undirected nature of Fermi fields, it is standard to represent them by undirected lines.

\section{Anomalies}

The generalized anomaly cancellation condition (6.2) becomes

$$
0=N_{\chi_{\text {in }}}+N_{\chi_{\text {out }}}-N_{F}-2 N_{\star},
$$

with $N_{\chi_{\text {in }}}, N_{\chi_{\text {out }}}, N_{F}$, and $N_{\star}$ the numbers of incoming chirals, outgoing chirals, and Fermis and the rank of the gauge group, respectively. A few words are in order for understanding this expression. First, we notice that the contributions of the incoming and outgoing chirals, i.e., of chirals transforming in the fundamental and antifundamental representations, have the same sign. This is because anomalies in $2 \mathrm{D}$ are quadratic. Second, unlike in the 4D case, there is a nonvanishing term proportional to $N_{\star}$. This is the contribution to the anomaly of gauginos in the vector multiplet. Finally, the contributions from chiral fields have a sign opposite to the ones of Fermis and vector multiplets. This is due to the opposite chirality of the fermions in these superfields. It is quite remarkable that all these details emerge from the simple requirement of periodicity under mutations.

\section{Potential}

The degree of the potential for $m=2$ is 1 . This means that all terms in the potential are of the general form shown on the left of Fig. 20; namely, they consist of a single Fermi field and an arbitrary number of chiral fields. The physical interpretation of such a potential term is interesting. In particular, following our previous discussion, a $c=1$ arrow connecting nodes $i_{1}$ and $i_{2}$ can be interpreted either as a Fermi field $\Lambda_{i_{1} i_{2}}$ or as a conjugate Fermi field $\bar{\Lambda}_{i_{2} i_{1}}$. The first possibility leads to a contribution to a so-called $J$-term, while the second option gives a contribution to an $E$-term:

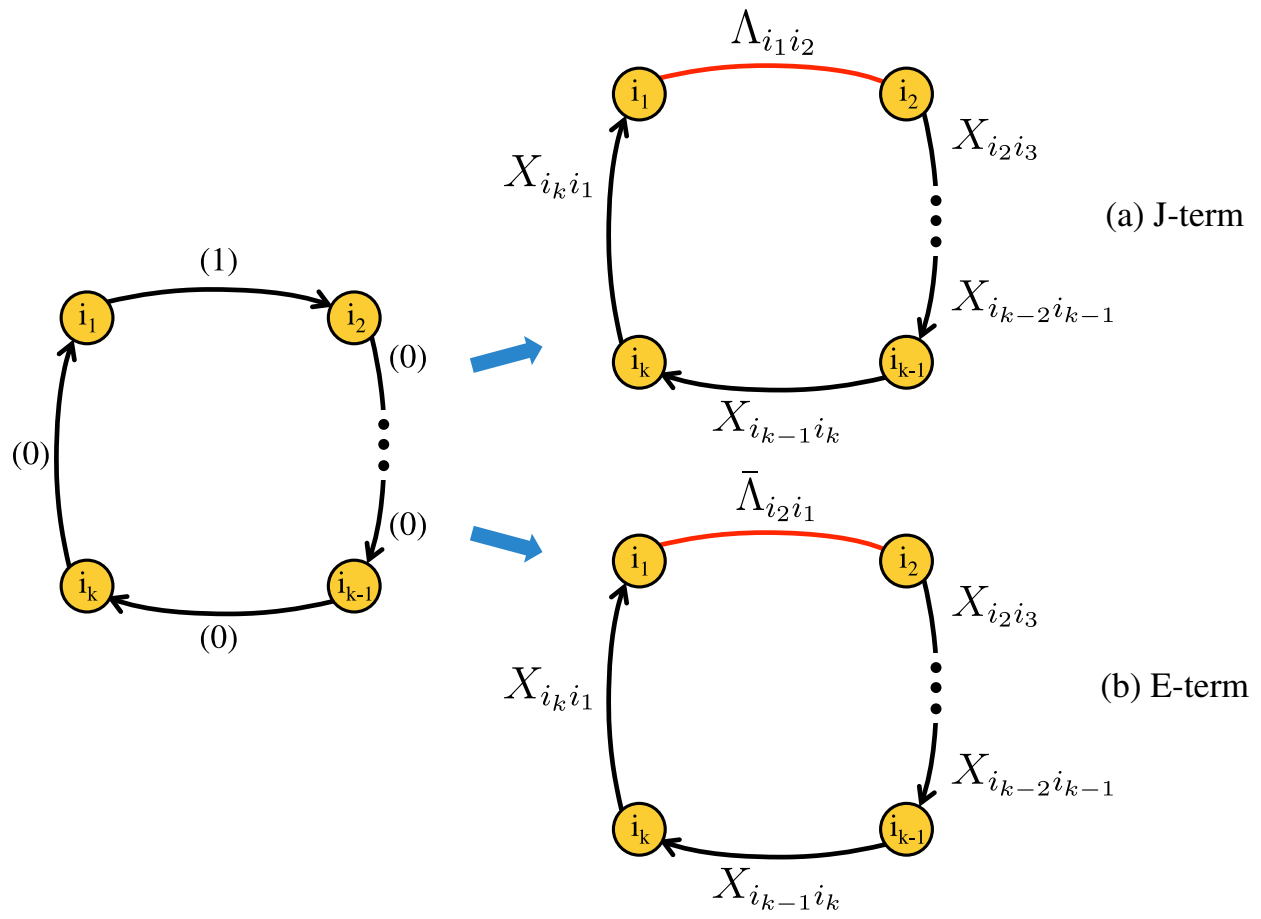

FIG. 20. There are two types of potential terms for $m=2$. They map to contributions to $J$ or $E$-terms in the corresponding $2 \mathrm{D}$ $\mathcal{N}=(0,2)$ gauge theories. 


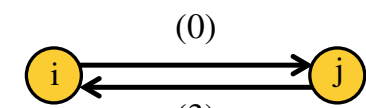

(3)

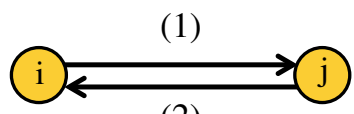

(2)
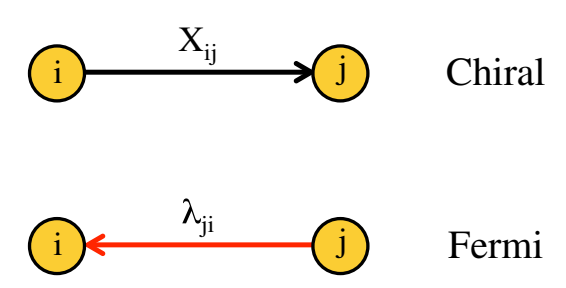

FIG. 21. (0,3) and $(1,2)$ arrows correspond to $0 \mathrm{D} \mathcal{N}=1$ chiral and Fermi fields, respectively.

$$
\varphi_{i_{1} i_{2}}^{(1)} \varphi_{i_{2} i_{3}}^{(0)} \ldots \varphi_{i_{k} i_{1}}^{(0)} \rightarrow\left\{\begin{array}{l}
J \text { term: } \Lambda_{i_{1} i_{2}} X_{i_{2} i_{3}} \ldots X_{i_{k} i_{1}} \\
E \text { term: } \bar{\Lambda}_{i_{2} i_{1}} X_{i_{2} i_{3}} \ldots X_{i_{k} i_{1}} .
\end{array}\right.
$$

More precisely, we refer to the chiral field parts of the cycles in (8.3) as $J$ and $E$-terms. Every Fermi field in the theory is associated to a $J$ and an $E$-term, which are given by sums over contributions that, generically, can be of different orders. For the terms in (8.3), we have

$$
\begin{aligned}
& J_{\Lambda_{i_{1} i_{2}}}=X_{i_{2} i_{3}} \ldots X_{i_{k} i_{1}}+\cdots \\
& E_{\Lambda_{i_{2} i_{1}}}=X_{i_{2} i_{3}} \ldots X_{i_{k} i_{1}}+\cdots,
\end{aligned}
$$

where the dots indicate possible additional terms.

The potential takes the form

$$
W=\sum_{a}\left[\Lambda_{a} J_{a}(X)+\bar{\Lambda}_{a} E_{a}(X)\right]
$$

where $a$ is an index that runs over all the Fermi fields in the theory.

The classical moduli space of the gauge theory requires vanishing $J$ and $E$-terms. This is in agreement with the discussion around (3.9), which states that only the Jacobian algebra with respect to arrows of degree $(m-1)$ is important for the moduli space. In this case, $m-1=1$, implying that we must consider the Jacobian algebra with respect to both Fermis and conjugate Fermis. In addition, as always, we demand vanishing $D$-terms.

\section{4. $\Lambda \leftrightarrow \bar{\Lambda}$ symmetry}

We have already mentioned that the unoriented nature of Fermi fields leads to a symmetry under the exchange of $\Lambda_{a} \leftrightarrow \bar{\Lambda}_{a}$ for any Fermi field. This symmetry corresponds to the exchange $J_{a} \leftrightarrow E_{a}$.

\section{Kontsevich bracket}

The potential (8.5) contains both Fermi fields and their conjugates. This implies that the vanishing of the Kontsevich bracket gives rise to a nontrivial constraint, which takes the form

$$
\sum_{a} J_{a}(X) E_{a}(X)=0
$$

This is precisely the trace condition of $2 \mathrm{D} \mathcal{N}=(0,2)$ theories [39].

\section{C. $m=3: 0 \mathrm{D} \mathcal{N}=1$}

Let us consider $m=3$ quivers. They correspond to $0 \mathrm{D}$ $\mathcal{N}=1$ gauge theories. These theories were recently studied in Ref. [3].

\section{Superfields}

Nodes correspond to gaugino superfields. There are two types of arrows, associated to $c=0,1$. The $(0,3)$ and $(1,2)$ double arrows map to chiral and Fermi superfields, respectively. We will refer to chiral fields as $X_{i j}$ and Fermi fields as $\lambda_{i j}$. Unlike what happens in the $m=2$ case, Fermi fields are oriented for $m=3$. The correspondence between double arrows and fields is illustrated in Fig. 21.

\section{Anomalies}

Anomaly cancellation becomes

$$
0=N_{\chi_{\text {in }}}-N_{\chi_{\text {out }}}+N_{F_{\text {in }}}-N_{F_{\text {out }}} .
$$

Fields transforming in the fundamental and antifundamental representations of the gauge group contribute with opposite signs. The orientation prescription of (2.4) is crucial for obtaining this correlation.

\section{Potential}

The potential for $m=3$ has degree 2. This implies that there are two possible types of potential terms, which precisely reproduce the possible interaction terms of $0 \mathrm{D}$ $\mathcal{N}=1$ gauge theories [3]. The first one, shown in Fig. 22, has the form

$$
J \text { term: } \varphi_{i_{1} i_{2}}^{(2)} \varphi_{i_{2} i_{3}}^{(0)} \ldots \varphi_{i_{k} i_{1}}^{(0)} \rightarrow \lambda_{i_{1} i_{2}} X_{i_{2} i_{3}} \ldots X_{i_{k} i_{1}}
$$

and corresponds to a contribution to a so-called $J$-term. $J$ terms are defined as the chiral field part of such loops. There is one $J$-term for every Fermi field, which becomes

$$
J_{\lambda_{i_{1} i_{2}}}=X_{i_{2} i_{3}} \ldots X_{i_{k} i_{1}}+\cdots,
$$

where the dots indicate the possibility of multiple contributions.

The second type of potential term is a contribution to an $\mathrm{H}$-term. It is shown in Fig. 23 and is given by 


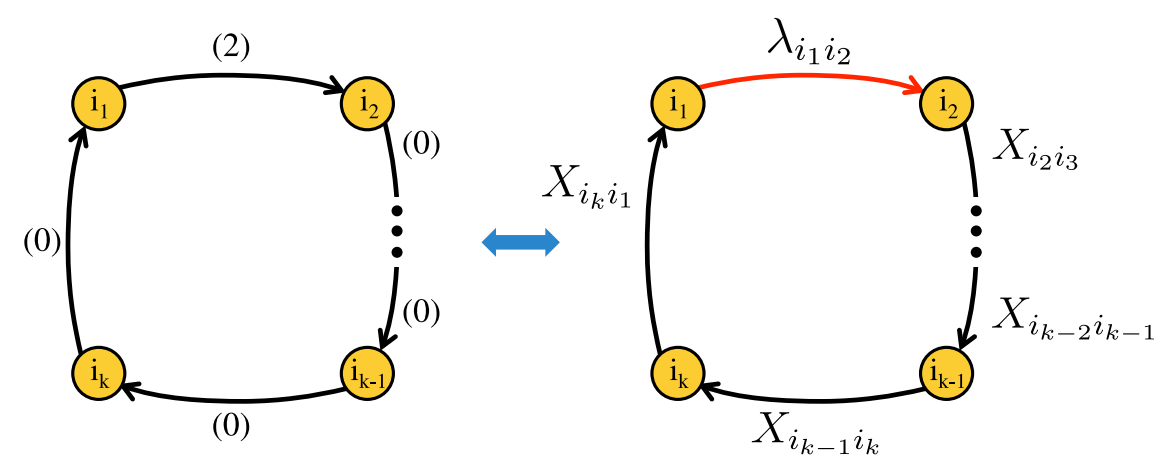

FIG. 22. Contribution to a $J$-term in a $0 \mathrm{D} \mathcal{N}=1$ gauge theory.

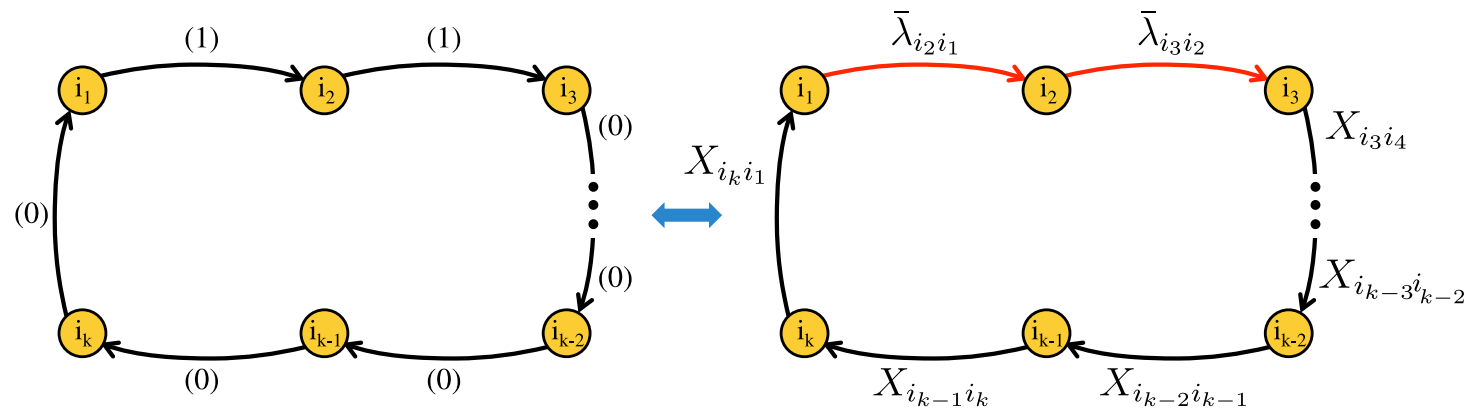

FIG. 23. Contribution to an $H$-term in a $0 \mathrm{D} \mathcal{N}=1$ gauge theory.

$H$ term: $\varphi_{i_{1} i_{2}}^{(1)} \varphi_{i_{2} i_{3}}^{(1)} \varphi_{i_{3} i_{4}}^{(0)} \ldots \varphi_{i_{k} i_{1}}^{(0)} \rightarrow \bar{\lambda}_{i_{2} i_{1}} \bar{\lambda}_{i_{3} i_{2}} X_{i_{3} i_{4}} \ldots X_{i_{k} i_{1}}$.

There is an $H$-term for every pair of Fermi fields. Once again, $H$-terms are defined in terms of the chiral fields in the loops. For (8.10), we have

$$
H_{\lambda_{i_{2} i_{1}} \lambda_{i_{3} i_{2}}}=X_{i_{3} i_{4}} \ldots X_{i_{k} i_{1}}+\cdots
$$

The full potential can be written in terms of $J$ and $H$ terms as

$$
W=\sum_{a} \lambda_{a} J_{a}+\sum_{a, b} \bar{\lambda}_{a} \bar{\lambda}_{b} H^{a b}
$$

where $a$ and $b$ run over Fermi fields.

In addition to vanishing $D$-terms, the moduli space of these theories is determined by only imposing vanishing $J$ terms. Once again, this is in full agreement with (3.9); namely, we only use the Jacobian algebra with respect to degree- $(m-1)$ arrows, which in this case are the $\lambda_{a}$ 's.

\section{Kontsevich bracket}

The potential is holomorphic in chiral fields but contains both Fermi fields and their conjugates. The vanishing of the Kontsevich bracket requires

$$
\sum_{a, b} \bar{H}^{a b}(\bar{X}) \bar{J}_{a}(\bar{X}) \lambda_{b}=0
$$

Since every $\lambda_{a}$ is independent, this condition becomes

$$
\sum_{b} \bar{H}^{a b}(\bar{X}) \bar{J}_{a}(\bar{X})=0 \text { for every } a .
$$

This is the $H$ constraint, which in physics is necessary for preserving SUSY [3].

It is striking that the mathematical formulation of SUSY gauge theories in different dimensions in terms of graded quivers with potentials provides a unified explanation for seemingly unrelated constraints on the potential, such as the trace condition in two dimensions and the $H$-constraint in zero dimensions.

\section{Comments on $m=0$ and $6 \mathrm{D} \mathcal{N}=(1,0)$}

Our discussion in previous sections started from $m=1$. This is a natural starting point since, mathematically, it corresponds to ordinary quivers and the first nontrivial example of mutations. However, our framework applies even for $m=0$, which becomes the natural initial case for the infinite tower of theories.

Extending the dictionary in Sec. VII, $m=0$ corresponds to $6 \mathrm{D} \mathcal{N}=(1,0)$ gauge theories. Such theories can be realized on the world volume of D5-branes probing CY 2folds. The case in which the $\mathrm{CY}_{2}$ is toric is particularly 


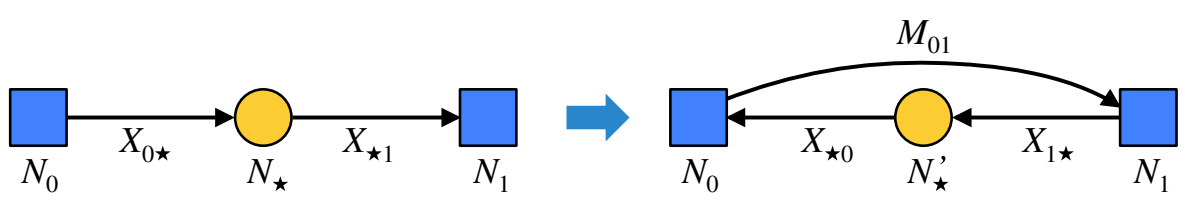

FIG. 24. The mutation of an $m=1$ quiver reproduces $4 \mathrm{D} \mathcal{N}=1$ Seiberg duality.

tractable. Toric $\mathrm{CY}_{2}$ 's can only be $\mathbb{C}^{2} / \mathbb{Z}_{n}$ orbifolds, for which the toric diagrams are given by segments of $n+1$ points in $\mathbb{Z}$ [41-44]. These setups are $T$ dual to so-called elliptic models consisting of stacks of D6-branes suspended from $n$ parallel NS5-branes on $S^{1}$ [44]. Elliptic models can be regarded as the simplest cousins of brane tilings. The corresponding necklace quivers realize the McKay correspondence for $\tilde{A}_{n-1}$ [45].

The interpretation of $m=0$ quivers as $6 \mathrm{D} \mathcal{N}=(1,0)$ gauge theories works as follows. Nodes correspond to vector multiplets, while $(0,0)$ unoriented arrows correspond to hypermultiplets. Six-dimensional $\mathcal{N}=(1,0)$ gauge theories need to be coupled to tensor multiplets in order to be anomaly free. For example, in the simple elliptic models mentioned above, there is one $(1,0)$ tensor multiplet for each NS5-brane. ${ }^{31}$ This in turn means that there is one tensor multiplet per gauge group factor. We conclude that within the class of theories considered in this paper nodes also represent the tensor multiplets. Since $m-1=-1$ in this case, these theories do not have a potential. Finally, there is no mutation, i.e., duality, in this case.

\section{MUTATIONS AS QFT DUALITIES}

For $m=1,2,3$ the mutations introduced in Sec. IV reproduce exactly the dualities of the corresponding quantum field theories. More precisely, for $m=1$, we obtain the Seiberg duality of 4D $\mathcal{N}=1$ theories [1]; for $m=2$, we recover the triality of $2 \mathrm{D}(0,2)$ theories [2]; and for $m=3$, we get the quadrality of $0 \mathrm{D} \mathcal{N}=1$ theories [3].

Seiberg duality is the prototypical and best-understood example of a SUSY quantum field theory duality. It has passed numerous tests and found countless applications. The discovery of triality is far more recent [2], and it was initially motivated by the invariance of the elliptic genus. By now, triality has been derived from Seiberg duality through compactification $[46,47]$ and realized in terms of branes $[6,9]$. Finally, quadrality was postulated based on mirror symmetry [3]. These dualities are beautifully unified when realized in terms of geometric transitions using mirror symmetry $[3,9,17]$. Remarkably, the theory of graded quivers with potentials and their mutations achieves a similar algebraic unification of dualities in different dimensions. The existence of this subjacent mathematical

\footnotetext{
${ }^{31}$ One of these tensor multiplets corresponds to the center-ofmass motion and is hence decoupled.
}

structure adds further credence to the recently proposed dualities. $^{32}$

Below, we show in simple examples how the mutation of graded quivers precisely reproduces the dualities of minimally supersymmetric gauge theories in $d=4,2,0$. In doing so, we illustrate the straightforward reformulation of the mutation rules in physics language. We will consider simple theories that can be regarded as generalizations of super QCD (SQCD) in various dimensions, consisting of a single gauge group and flavors of all $m+1$ possible types.

In the rest of this section, circular and square nodes correspond to gauge and global nodes, respectively. The ranks of the different flavor nodes are denoted $N_{i}$, where $i=0, \ldots, m$ is the degree of the corresponding field regarded as an arrow going into the gauge node as in Fig. 4. $N_{\star}$ is the original rank of the gauge group.

\section{A. $m=1: 4 \mathrm{D} \mathcal{N}=1$ Seiberg duality}

Let us first consider $m=1$. Our starting point is standard 4D SQCD, the quiver of which is shown on the left of Fig. 24. It contains two flavor nodes, which correspond to incoming and outgoing chiral fields at the gauge node. The anomaly cancellation condition (6.2) requires $N_{0}=N_{1}$, which is often referred to as the number of flavors $N_{F}$. Similarly, $N_{\star}$ is usually denoted the number of colors $N_{c}$.

According to the general rules in Sec. IV A, mutating the gauge node transforms the quiver as follows:

(i) Flavors.-Figure 4 summarizes the transformation of flavors for general $m$ in terms of a rotation. In this simple case, this rotation reduces to reversing the orientation of the flavor arrows.

(ii) Mesons.-According to rule 2, the dual theory contains a meson that, expressed in terms of the fields in the original theory, is given by $M_{01}=X_{0 \star} X_{\star 1}$.

The final quiver is shown on the right-hand side of Fig. 24. Following (4.1), the new rank of the gauge group is $N_{\star}^{\prime}=N_{0}-N_{\star}$. Finally, rule 2.a implies that the final theory contains the superpotential

$$
W=M_{01} X_{1 \star} X_{\star 0} .
$$

We conclude that for $m=1$ the mutation introduced in Sec. IV precisely coincides with Seiberg duality.

\footnotetext{
${ }^{32}$ The term "duality" might sound like a misnomer since we are referring to transformations that are not involutions, but this nomenclature has become standard in physics, so we adhere to it.
} 

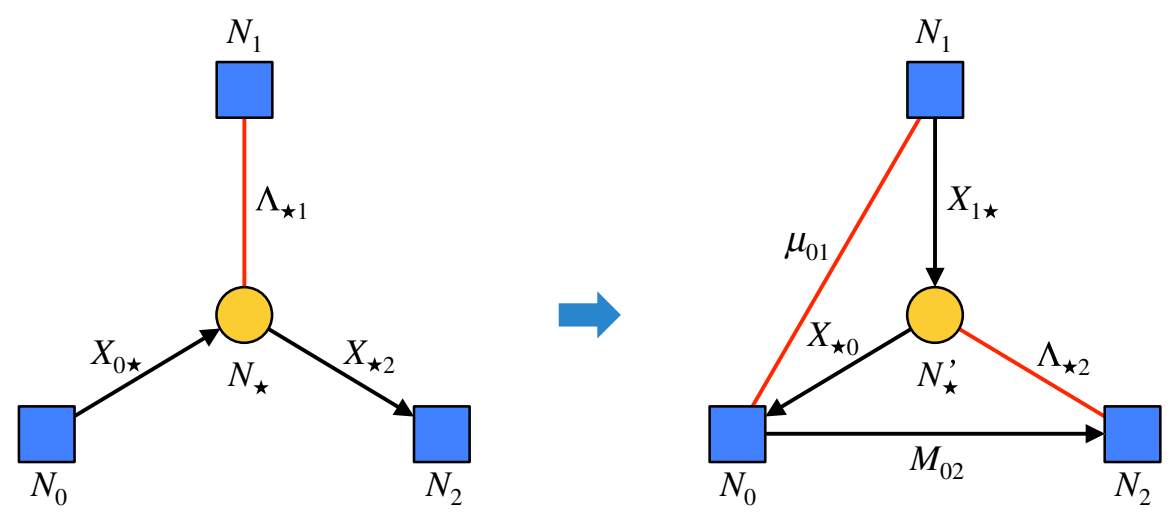

FIG. 25. The mutation of an $m=2$ quiver reproduces $2 \mathrm{D} \mathcal{N}=(0,2)$ triality.
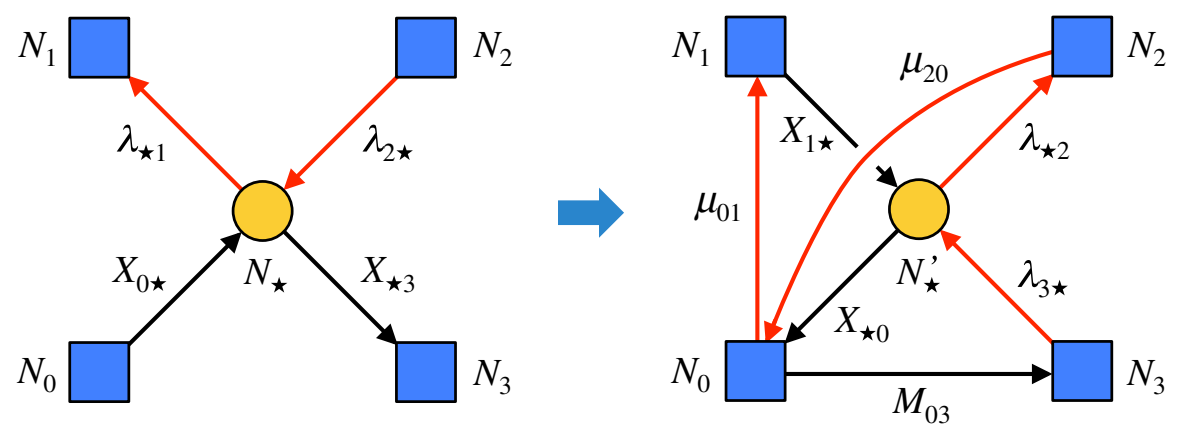

FIG. 26. The mutation of an $m=3$ quiver reproduces $0 \mathrm{D} \mathcal{N}=1$ quadrality.

\section{B. $m=2: 2 \mathrm{D} \mathcal{N}=(0,2)$ triality}

We now consider the $2 \mathrm{D}(0,2)$ theory on the left of Fig. 25. It contains three flavor nodes, corresponding to incoming chirals, outgoing chirals, and Fermis. Anomaly cancellation (6.2) requires

$$
N_{\star}=\frac{N_{0}-N_{1}+N_{2}}{2} .
$$

Mutating the gauge node, the quiver transforms as follows:

(i) Flavors. - The rotation of flavors in Fig. 4 becomes

$$
\begin{aligned}
\text { chiral in } & \rightarrow \text { chiral out } \\
\text { Fermi } & \rightarrow \text { chiral in } \\
\text { chiral out } & \rightarrow \text { Fermi. }
\end{aligned}
$$

(ii) Mesons.-Following rule 2, the incoming chirals can be composed with the outgoing chirals or the Fermis, giving rise to two mesons,

$$
\begin{aligned}
M_{02}=X_{0 \star} X_{\star 2} & (\text { chiral }) \\
\mu_{01}=X_{0 \star} \Lambda_{\star 1} & \text { (Fermi }),
\end{aligned}
$$

where we have indicated the type of superfield. The resulting quiver is presented on the right of Fig. 25.
Once again, the rank of the gauge group in the dual theory is $N_{\star}^{\prime}=N_{0}-N_{\star}$. Following rule 2.a, the potential of the dual theory consists of the cubic dual flavors-meson couplings

$$
W=\bar{\Lambda}_{\star 2} X_{\star 0} M_{02}+\mu_{01} X_{1 \star} X_{\star 0},
$$

where the first term corresponds to an $E$-term and the second one corresponds to a $J$-term. Since each term involves a different Fermi field, the trace condition (8.6) is trivially satisfied.

In summary, for $m=2$ the mutation in Sec. IV coincides with triality.

\section{C. $m=3: 0 d \mathcal{N}=1$ quadrality}

Finally, let us study the $0 \mathrm{D} \mathcal{N}=1$ theory on the left of Fig. 26. This model was originally studied in Ref. [3]. Anomaly cancellation (6.2) requires

$$
N_{0}-N_{1}+N_{2}-N_{3}=0
$$

Mutating the gauge node, the transformation of the quiver is given by: 
(i) Flavors.-From Fig. 4, the flavors mutate according to

$$
\begin{aligned}
\text { chiral in } & \rightarrow \text { chiral out } & \text { Fermi in } & \rightarrow \text { Fermi out } \\
\text { Fermi out } & \rightarrow \text { chiral in } & \text { chiral out } & \rightarrow \text { Fermi in. }
\end{aligned}
$$

(ii) Mesons.-From rule 2, we obtain three mesons:

$$
\begin{aligned}
& M_{03}=X_{0 \star} X_{\star 3} \quad \text { (chiral) } \\
& \mu_{01}=X_{0 \star} \lambda_{\star 1} \quad \text { (Fermi) } \\
& \mu_{20}=\lambda_{2 \star} \bar{X}_{0 \star} \quad \text { (Fermi). }
\end{aligned}
$$

The meson $\mu_{20}$ results from the composition between a Fermi field and a conjugate chiral field and is thus an example of anticomposition. Indeed, anticomposition was first observed in physics in the context of quadrality [3]. In view of the results of this paper, this is not surprising, since zero dimensions corresponds to $m=3$, which is the first nontrivial instance of anticomposition. ${ }^{33}$ In Ref. [3], evidence for anticomposition came from multiple fronts, including the transformation of brane charges under a geometric transition, the matching of anomalies and deformations between dual theories, and the periodicity under four consecutive dualizations. The emergence of anticomposition from a simple mathematical structure is reassuring.

The final quiver is presented on the right-hand side of Fig. 26. The rank of the gauge group in the dual theory is $N_{\star}^{\prime}=N_{0}-N_{\star}$. As in the two previous examples, since the original theory has no potential, the final potential follows entirely from rule 2 . $a$ and is given by

$$
W=\mu_{01} X_{1 \star} X_{\star 0}+\lambda_{3 \star} X_{\star 0} M_{03}+\bar{\mu}_{20} \bar{\lambda}_{\star 2} X_{\star 0} .
$$

The first two terms correspond to $J$-terms, while the last one corresponds to an $H$-term. The $H$-constraint (8.14) is trivially satisfied.

We conclude that for $m=3$ the mutation in Sec. IV reproduces quadrality.

We conclude this section with some comments regarding the mutation of potentials. The basic physics principle for determining the potentials of dual theories is that every term that is allowed by the symmetries of a theory must be present. The rules for mutating the potential introduced in Sec. IV B beautifully implement this principle. While the prescription based on symmetries is absolutely general, it can become hard to apply in complicated theories. The rules of Sec. IV B are local; namely, they focus on the modification of the quiver in the neighborhood of

\footnotetext{
${ }^{33} \mathrm{We}$ can think that anticomposition is also present for $m=2$, but it acts rather trivially due to the Fermi-conjugate Fermi symmetry.
}

the mutated node and are hence much more practical. In fact, they can be automatically implemented in a computer. Before this work, such local rules were only known for Seiberg duality $(m=1)$. For triality, the most detailed understanding of the mutation of potentials was attained for toric theories [4-7,9]. Even in this class of theories, the potential of dual theories must be read off from periodic quivers or brane brick models, and doing so can become quite challenging. For $0 \mathrm{D} \mathcal{N}=1$ theories, local rules for the transformation of the potential under quadrality are just not known. The rules in Sec. IV B hence represent a significant development in our understanding on how potentials mutate in four dimensions, two dimensions, and zero dimensions. The list of known explicit examples in two dimensions and zero dimensions is still limited but rapidly growing (see, e.g., Refs. [3-9]). It is indeed possible to verify that our prescription reproduces all of them.

\section{MIRROR SYMMETRY: GRADED QUIVERS FOR TORIC CALABI-YAUS}

In this section, we focus on toric CY $(m+2)$-folds, which give rise to a particularly nice family of $m$-graded quivers. We have already seen hints of this class of theories in Secs. VII and VIII, in which we discussed D-branes probing these geometries for $m=0,1,2,3$. A powerful way for connecting toric geometry to quivers involves mirror symmetry. Our primary goal is to emphasize that, while this construction has a D-brane interpretation for $m \leq 3$, it actually applies to arbitrary $m$. Below, we present a brief review of the key ideas and refer the reader to Refs. [3,9,48,49] for further details.

A toric $\mathrm{CY}_{m+2} \mathcal{M}$ is specified by a toric diagram $V$, which is a convex set of points in $\mathbb{Z}^{m+1}$. The corresponding mirror geometry $[50,51]$ is an $(m+2)$-fold $\mathcal{W}$ defined as a double fibration over the complex $W$ plane,

$$
\begin{aligned}
& W=P\left(x_{1}, \ldots, x_{m+1}\right) \\
& W=u v,
\end{aligned}
$$

where $u, v \in \mathbb{C}$ and $x_{\mu} \in \mathbb{C}^{*}, \mu=1, \ldots, m+1 . P\left(x_{1}, \ldots, x_{m+1}\right)$ is the Newton polynomial, which is defined as

$$
P\left(x_{1}, \ldots, x_{m+1}\right)=\sum_{\vec{v} \in V} c_{\vec{v}} x_{1}^{v_{1}} \ldots x_{n-1}^{v_{m+1}},
$$

where the $c_{\vec{v}}$ are complex coefficients and the sum is over points $\vec{v}$ in the toric diagram. We can set $m+2$ of the coefficients to 1 by rescaling the $x_{\mu}$ 's.

The critical points of $P$ are given by $\left(x_{1}^{*}, \ldots, x_{m+1}^{*}\right)$ satisfying

$$
\left.\frac{\partial}{\partial x_{\mu}} P\left(x_{1}, \ldots, x_{m+1}\right)\right|_{\left(x_{1}^{*}, \ldots, x_{m+1}^{*}\right)}=0 \quad \forall \mu .
$$


The corresponding critical values on the $W$ plane are $W^{*}=P\left(x_{1}^{*}, \ldots, x_{m+1}^{*}\right)$. For toric diagrams with at least one internal point, it can be shown that the number of critical points of $P$, which we call $G$, is equal to the normalized volume of the toric diagram [48]. In more detail, the normalization is determined with respect to the volume of a minimal "tetrahedron" in $(m+1)$ dimensions.

The double fibration consists of a holomorphic $m$ complex-dimensional surface $\Sigma_{W}$ coming from $P\left(x_{1}, \ldots, x_{m+1}\right)$ and a $\mathbb{C}^{*}$ fibration from $u v$. The corresponding $S^{m} \times S^{1}$ is fibered over a vanishing path, which is a line segment connecting $W=0$ and $W=W^{*}$, and gives rise to an $S^{m+2}{ }^{34}$ We refer to these spheres as $\mathcal{C}_{i}, i=1, \ldots, G$.

The $\mathcal{C}_{i}$ are in one-to-one correspondence with vanishing cycles $C_{i}$ at $W=0$, where the $S^{1}$ fiber vanishes. Every $\mathcal{C}_{i}$ gives rise to a vanishing cycle $C_{i}$ with $S^{m+1}$ topology. The $C_{i}$ live on the Riemann surface $\Sigma_{0}$, defined by $P\left(x_{1}, \ldots, x_{m+1}\right)=0$. As we explain below, the quiver theory is determined by how the $C_{i}$ 's intersect.

\section{A. Tomography}

A convenient way of visualizing the geometry of the $S^{m}$ 's is in terms of tomography $[9,49]$. The $x_{\mu}$ tomography corresponds to the projection of the $S^{n-2}$ spheres at $W=0$ onto the $x_{\mu}$ plane. An attractive feature of tomography is that it is easy to scale: every time $m$ is increased by 1 , we just include an additional $x_{\mu}$ plane. Various explicit examples of tomography for $m=1$ and 2 can be found in Ref. [9].

\section{From mirror symmetry to quivers}

The quiver diagram can be read from the mirror geometry. In fact, the mirror geometry specifies the full theory, namely, not only its quiver but also its potential. The theories associated to toric CY $(m+2)$-folds are fully encoded by periodic quivers living on an $(m+1)$ dimensional torus $\mathbb{T}^{m+1}$. All the terms in the potential are mapped to minimal plaquettes in the periodic quiver. We refer the reader to Refs. [3-5,35] for implementations of this construction to $m \leq 3$. In this section, we outline the basics of the map between mirror geometries and quiver theories. A detailed study of the quiver theories associated to toric CY $(m+2)$-folds for arbitrary $m$ will be presented elsewhere [52].

Every vanishing cycle $C_{i}$ corresponds to a node in the quiver. According to our previous discussion, the number of nodes is then equal to the normalized volume of the toric diagram.

Every intersection between vanishing cycles gives rise to a field in the quiver. Depending on the coefficients in the Newton polynomial, intersections might not be fully

\footnotetext{
${ }^{34}$ More precisely, vanishing paths can be curved. See Ref. [9] for a discussion.
}

resolved; i.e., they might have higher multiplicity. Explicit examples of this situation are studied in Ref. [9].

Finally, the periodic quiver is obtained by taking the coamoeba projection

$$
\left(x_{1}, \ldots, x_{m+1}\right) \mapsto\left(\arg \left(x_{1}\right), \ldots, \arg \left(x_{m+1}\right)\right),
$$

which maps the intersections between vanishing cycles to the positions of the corresponding fields on the torus $\mathbb{t}^{m+1}$.

Example: $\mathbb{C}^{6} / \mathbb{Z}_{6}$.-To illustrate these ideas, let us briefly consider the $\mathbb{C}^{6} / \mathbb{Z}_{6}$ orbifold with action $(1,1,1,1,1,1)$. The toric diagram for this geometry is given by the following collection of points in $\mathbb{Z}^{5}$ :

$$
\begin{array}{cc}
(1,0,0,0,0) & \\
(0,1,0,0,0) & (0,0,0,0,0) \\
\vdots & (-1,-1,-1,-1,-1) \\
(0,0,0,0,1) &
\end{array}
$$

Six of the coefficients of the corresponding Newton polynomial can be scaled to 1, leaving a single free coefficient. In the notation of (10.2), we pick this coefficient to be $c_{(0,0,0,0,0)}$. Setting it to zero, we obtain

$P\left(x_{1}, x_{2}, x_{3}, x_{4}, x_{5}\right)=x_{1}+x_{2}+x_{3}+x_{4}+x_{5}+\frac{1}{x_{1} x_{2} x_{3} x_{4} x_{5}}$.

The normalized volume of the toric diagram defined by (10.6) is 6. This leads to six critical points of $P$, which in turn map to six nodes in the quiver, as expected for a $\mathbb{C}^{6} / \mathbb{Z}_{6}$ orbifold. The critical values are $W_{j}^{*}=6 \omega^{j}$, with $\omega=e^{i \pi / 3}$ and $j=1, \ldots, 6$. Figure 27 shows the vanishing paths on the $W$ plane and the $x_{1}$ tomography.

The quiver theory associated to this geometry has $m=4$. The theory has an $S U(6)$ global symmetry corresponding to the isometry of the orbifold. ${ }^{35}$ The quiver diagram consists of six nodes and arrows $\Phi_{i, i+c+1}^{(c)}, c=0,1,2$, transforming in the $(c+1)$-index antisymmetric representation of $S U(6)$. These properties generalize straightforwardly to $\mathbb{C}^{m+2} / \mathbb{Z}_{m+2}$ orbifolds with action $(1, \ldots, 1)$, as will be explained in Ref. [18]. The $\Phi_{i, i+3}^{(2)}$ fields are unoriented.

Figure 28 shows the quiver diagram for this theory. Black, red, and purple lines correspond to degrees 0,1 ,

\footnotetext{
${ }^{35}$ Notice that the $S U(6)$ global symmetry follows from the fact that we picked an orbifold that has the same action on each complex plane. Other $\mathbb{C}^{6} / \mathbb{Z}_{6}$ orbifolds have different global symmetries.
} 

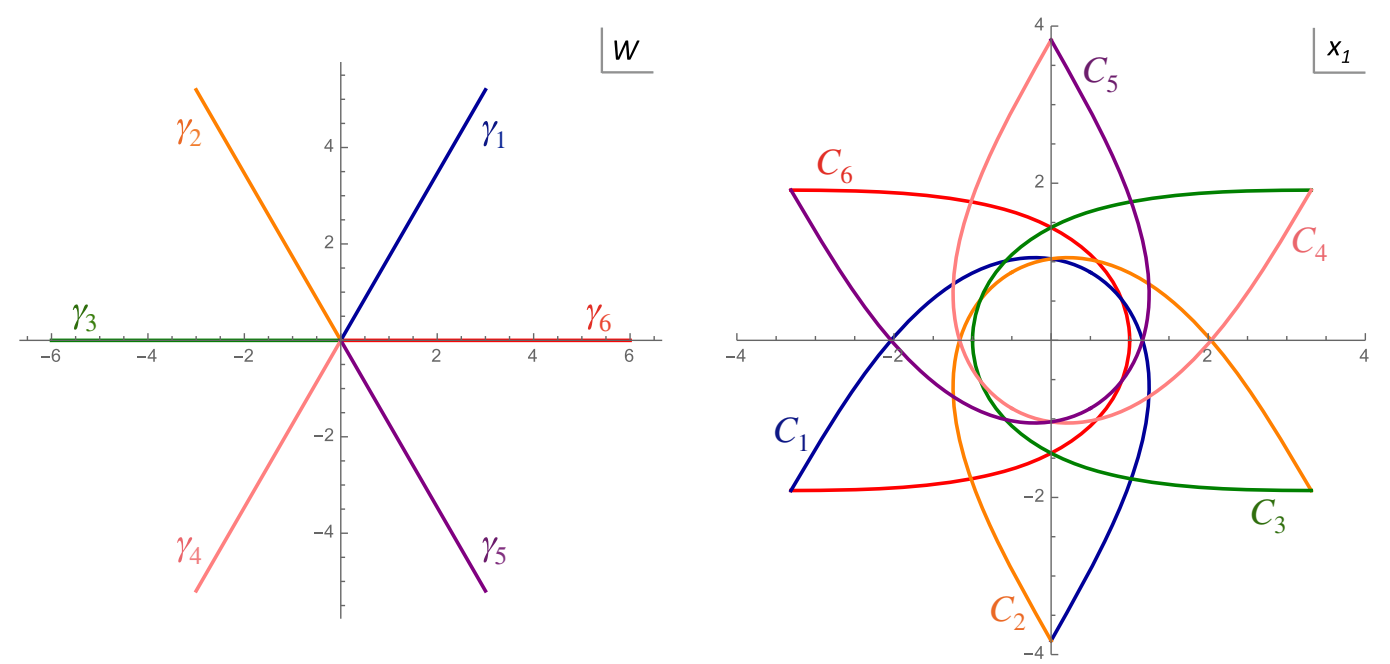

FIG. 27. Vanishing paths and $x_{1}$ tomography for the $\mathbb{C}^{6} / \mathbb{Z}_{6}$ orbifold with action $(1,1,1,1,1,1)$.

and 2, respectively. We also indicate the $S U(6)$ representation in which each type of field transforms.

The potential is purely cubic and takes the form

$$
\begin{aligned}
W= & \sum_{i=1}^{6} \epsilon_{a_{1} \ldots a_{6}}\left[\epsilon^{a_{3} \ldots a_{8}} \Phi_{i, i+1}^{a_{1}(0)} \Phi_{i+1, i+2}^{a_{2}(0)} \bar{\Phi}_{a_{7} a_{8} ; i, i+2}^{(1)}\right. \\
& +\Phi_{i, i+1}^{a_{1}(0)} \Phi_{i+1, i+3}^{a_{2} a_{3}(1)} \Phi_{i+3, i}^{a_{4} a_{5} a_{6}(2)} \\
& +\epsilon^{a_{4} \ldots a_{9}} \Phi_{i, i+1}^{a_{1}(0)} \Phi_{i+1, i+3}^{a_{2} a_{3}(1)} \bar{\Phi}_{a_{7} a_{8} a_{9} ; i, i+3}^{(2)} \\
& \left.+\Phi_{i, i+2}^{a_{1} a_{2}(1)} \Phi_{i+2, i+4}^{a_{3} a_{4}(1)} \Phi_{i+4, i}^{a_{5} a_{6}(1)}\right] .
\end{aligned}
$$

The bifundamental indices are taken $\bmod (6)$. The $a_{\mu}$ superscripts and subscripts are $S U(6)$ fundamental and antifundamental indices, respectively. They are contracted such that the potential is $S U(6)$ invariant. This is the first example of a graded quiver theory associated to a toric $\mathrm{CY}_{6}$ to appear in the literature.

Mirror symmetry and degree.-So far, we have discussed how every intersection between vanishing cycles gives rise

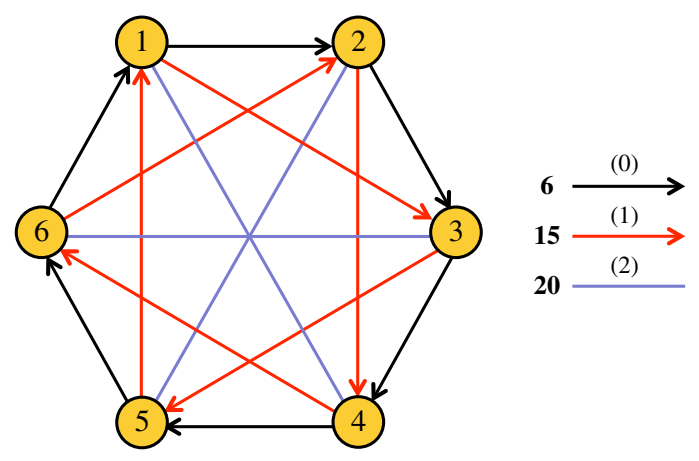

FIG. 28. Quiver diagram for the $\mathbb{C}^{6} / \mathbb{Z}_{6}$ orbifold with action $(1,1,1,1,1,1)$. to a field in the quiver. We have not, however, explained how to determine the corresponding degree. Understanding this is a crucial ingredient for completing the map between the mirror geometry and graded quivers.

This question has been already addressed for the cases of $m=1$ and 2. For $m=1$, the two possible degrees correspond to the two orientations of chiral fields and follow directly from the orientation of the intersecting cycles. This prescription is equivalent to the one based on the directions of intersecting zigzag paths on brane tilings $[48,53]$. For $m=2$, the degree can also be determined by a detailed analysis of the intersection [9]. An alternative systematic approach consists of connecting the geometry of interest to an orbifold by partial resolution. It is straightforward to determine field degrees for orbifolds and to follow them through the process of partial resolution. This method has been successfully exploited for $m=1$ and 2 (see, e.g., Refs. [4,31-34] and references therein) and will be studied for general $m$ in Ref. [52].

Let us mention a general connection between the degree and the mirror. Consider an arbitrary vanishing cycle $C_{\star}$. Other vanishing cycles intersecting with $C_{\star}$ provide flavors to the corresponding node. For $m=1,2$, and 3 , it has been observed that the corresponding vanishing paths are arranged on the $W$ plane according to the cyclic order of increasing degree discussed in Sec. II A. The cyclic order emerges from geometry. This is more than an empirical observation; as we explain in the coming section, it is at the heart of the geometric realization of Seiberg duality, triality, and quadrality in terms of geometric transitions in the mirror $[3,9,17]$. We thus expect this property, which is schematically shown in Fig. 29, to hold for arbitrary $m$.

It is reasonable to expect that a prescription for reading the degree of an intersection directly from the mirror might exist. We leave this interesting question for future work. Note that the tomography of the mirror of a $\mathrm{CY}_{m+2}$ 


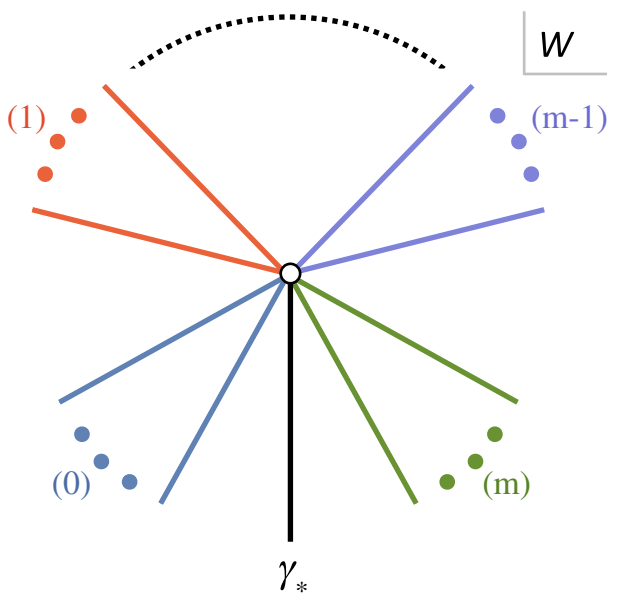

FIG. 29. For any reference cycle, the other vanishing paths are degree ordered on the $W$ plane.

involves $(m+1) x_{i}$ planes. This is precisely the same number of possible degrees in the corresponding quiver.

\section{Mirror symmetry and mutations}

For completeness, let us briefly discuss how the mutations of Sec. IV are realized as geometric transitions in the mirror geometry. This understanding was developed for $m=1$ in Ref. [17], for $m=2$ in Ref. [9], and for $m=3$ in Ref. [3]. Indeed, in Ref. [3], it was emphasized that mirror symmetry provides a unification of the mutations for different values of $m$. The previous works focused on the physically understood instances of $m \leq 3$. Our main new statement is that this geometric implementation of mutations applies to arbitrary $m$.

The mutation of a node in the quiver associated to the vanishing cycle $C_{*}$ corresponds to the geometric transition shown in Fig. 30. The moduli of the underlying CY $(m+2)$-fold are changed until the vanishing path $\gamma_{\star}$ moves past all the degree- 0 vanishing paths, i.e., those

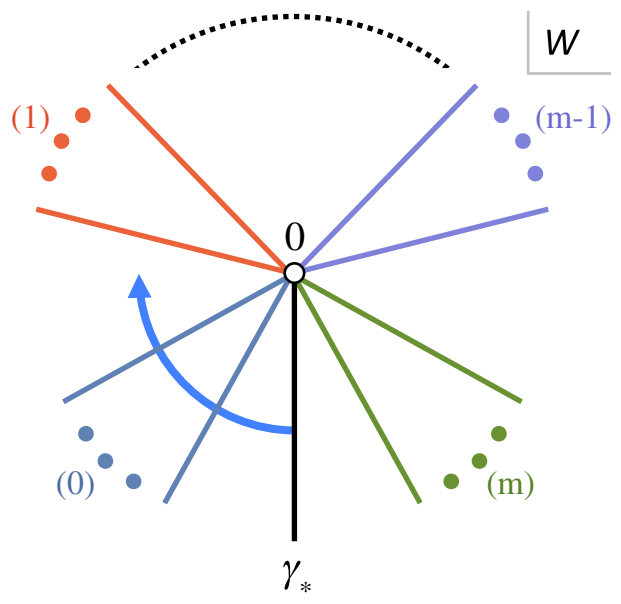

FIG. 30. A mutation is realized as a geometric transition in the mirror. contributing incoming chirals to the mutated node, on the $W$ plane.

Figure 30 makes it clear that the mutation is an order- $(m+1)$ operation, since the vanishing paths associated to different degrees divide the $W$ plane into $(m+1)$ wedges.

\section{ALGEBRAIC DIMENSIONAL REDUCTION}

In physics, dimensional reduction is the process that starts from a gauge theory in $d$ dimensions, assumes that fields are independent of $\Delta d$ of them, and results in a new gauge theory in $d^{\prime}=d-\Delta d$ dimensions. The relevant cases for this paper are the $6 \mathrm{D} \rightarrow 4 \mathrm{D}, 4 \mathrm{D} \rightarrow 2 \mathrm{D}$, and $2 \mathrm{D} \rightarrow 0 \mathrm{D}$ reductions of minimally supersymmetric gauge theories. ${ }^{36}$ Each of them decreases the dimension $d \rightarrow d^{\prime}=d-2$ while increasing $m \rightarrow m^{\prime}=m+1$. From the perspective of the number of dimensions in which the gauge theory lives, it is clear that dimensional reduction cannot proceed beyond $m=3$, since it would require us to go below zero dimensions. We instead focus on the corresponding CY. Dimensional reduction increases the dimension of the $\mathrm{CY}$ by 1 in a very special way, simply adding a $\mathbb{C}$ factor to the original geometry. The underlying geometry, i.e., the moduli space, thus changes as follows:

$$
\mathrm{CY}_{m+2} \rightarrow \mathrm{CY}_{m+3}=\mathrm{CY}_{m+2} \times \mathbb{C} \text {. }
$$

It is natural to adopt (11.1) as the definition of algebraic dimensional reduction acting on graded quivers, or dimensional reduction for short. ${ }^{37}$ This procedure coincides with dimensional reduction for the physical cases and generalizes it to arbitrary $\mathrm{m}^{38} \mathrm{~A}$ more appropriate name for this operation would perhaps be $C Y$ dimensional increase.

We now explain how this generalized notion of dimensional reduction admits an elegant implementation within the framework of graded quivers.

\section{A. Quiver}

Let us first discuss how the quiver transforms under dimensional reduction. We use the notation of (2.4) for arrows. To facilitate their distinction, we refer to the fields in the original theory as $\Phi$ and those in the dimensionally reduced theory as $\Psi$. Dimensional reduction is given by

\footnotetext{
${ }^{36}$ It is important to note that the dimensional reduction of a minimally supersymmetric theory is not minimally supersymmetric, but it has twice the minimal amount of SUSY in the lower dimension. Despite the extended SUSY, dimensionally reduced theories can be written in minimal SUSY language, which is what we do in this section.

${ }^{37}$ We will refer to this procedure simply as dimensional reduction for brevity and to emphasize the connection to the standard case.

${ }^{38}$ In the type-IIB string theory constructions discussed in Sec. VII, the upper bound on the dimension of the CY is 5. This follows from the fact that the ambient space is ten dimensional.
} 


$$
\begin{array}{ccc}
m & & m+1 \\
\operatorname{node}_{i} & \rightarrow & \operatorname{node}_{i}+\operatorname{adjoint~chiral}_{i i}^{(0)} \\
\Phi_{i j}^{(c)} & \rightarrow & \Psi_{i j}^{(c)}+\tilde{\Psi}_{i j}^{(c+1)}
\end{array}
$$

where $0 \leq c \leq m / 2$. The previous table also applies when $i=j$, namely, when the starting theory contains adjoint fields. Notice that, modulo the new $\Psi_{i i}^{(0)}$ fields, this procedure preserves the chiral field content of the theory.

Equation (11.2) fully determines the dimensional reduction of a quiver. It is interesting to consider the undirected fields of degree $m / 2$ that can be present in theories with even $m$ in further detail. According to (11.2), we get

$$
\frac{\text { even } m}{\Phi_{i j}^{(m / 2)} \rightarrow \quad \Psi_{i j}^{(m / 2)}+\tilde{\Psi}_{i j}^{(m / 2+1)}=\Psi_{i j}^{(m / 2)}+\tilde{\Psi}_{j i}^{(m / 2)},}
$$

where we have used the fact that $\tilde{\Psi}_{i j}^{(m / 2+1)}=\tilde{\Psi}_{j i}^{(m / 2)}$. We went from a degree greater than $m^{\prime} / 2$ to a degree smaller than $m^{\prime} / 2$ by reversing the orientation of the arrow. In summary, the undirected fields $\Psi_{i j}^{(m / 2)}$ are dimensionally reduced to a pair of fields of degree $m / 2$ with opposite orientations.

\section{B. Potential}

Let $W_{m}$ denote the original potential, and let $W_{m+1}$ be the one for the dimensionally reduced theory. There are two types of contributions to $W_{m+1}$ :

(1) Dimensional reduction of terms in $W_{m}$.-The degree of the potential increases by 1 , going from $m-1$ in the parent theory to $m^{\prime}-1=m$ in the dimensionally reduced one. According to (11.2), every arrow $\Phi_{i j}^{(c)}$ in the initial theory gives rise to a pair of arrows $\Psi_{i j}^{(c)}+\tilde{\Psi}_{i j}^{(c+1)}$. The dimensional reduction of $W_{m}$ is then straightforward. For every term in $W_{m}$, we replace every field with the corresponding $\Psi_{i j}^{(c)}$ except for one, which we instead replace by $\tilde{\Psi}_{i j}^{(c+1)}$. We repeat this process for all fields in the term. This procedure generates a series of terms in $W_{m+1}$ for every term in $W_{m}$.

Schematically, for any term in $W_{m}$, we have

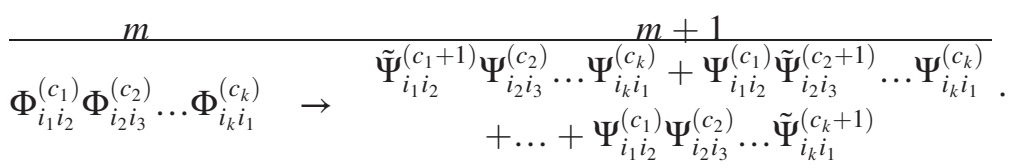

(2) New terms involving adjoints.-In addition, $W_{m+1}$ contains a new class of terms. For every arrow $\Phi_{i j}^{(c)}$ in the original quiver, we introduce the pair of potential terms in the dimensionally reduced one,

$$
\Psi_{i i}^{(0)} \Psi_{i j}^{(c)} \tilde{\Psi}_{j i}^{(m-c-1)}-\tilde{\Psi}_{j i}^{(m-c-1)} \Psi_{i j}^{(c)} \Psi_{j j}^{(0)},
$$

which uses the adjoint chiral fields arising from the dimensional reduction of every node. ${ }^{39}$ The choice of relative sign is a convention.

These two steps generate all possible terms in $W_{m+1}$ that are consistent with the symmetries of the theory.

It is straightforward to check that the prescription introduced in this section reproduces the $6 \mathrm{D} \rightarrow 4 \mathrm{D}$, $4 \mathrm{D} \rightarrow 2 \mathrm{D}$, and $2 \mathrm{D} \rightarrow 0 \mathrm{D}$ dimensional reduction of minimally supersymmetric gauge theories.

\section{Dimensional reduction of the moduli space}

We now explain how, under dimensional reduction, the moduli space transforms simply as in (11.1). For simplicity, let us restrict to theories in which the ranks of all nodes are

\footnotetext{
${ }^{39}$ These fields should not be confused with other adjoint fields that descend from preexisting adjoints in the initial theory.
}

equal to 1 . Following the discussion in Sec. III C, for computing the moduli space, we should focus exclusively on the chiral fields. Let us first consider the adjoint chiral fields $\Psi_{i i}^{(0)}$ descending from nodes in the parent theory. They only appear in the potential $W_{m+1}$ through the terms (11.5). The relations arising from the cyclic derivatives of those terms with respect to either $\Psi_{i j}^{(c)}$ or $\tilde{\Psi}_{j i}^{(m-c-1)}$ imply that $\Psi_{i i}^{(0)}=\Psi_{j j}^{(0)}$ for any $i$ and $j$. All of the $\Psi_{i i}^{(0)}$, s are thus equal and give rise to the decoupled $\mathbb{C}$ factor in (11.1). Next, we know that the chiral fields in the original theory $\Phi_{i j}^{(0)}$ subject to vanishing $D$-terms and the relations coming from the initial potential $W_{m}$ correspond to the geometry that we call $\mathrm{CY}_{m+2}$ in (11.1). According to (11.2), every such chiral gives rise to a chiral $\Psi_{i j}^{(0)}$ in the dimensionally reduced theory. The $D$-terms remain the same. Furthermore, the potential terms in (11.4) guarantee that the relations for the $\Psi_{i j}^{(0)}$, s that follow from $W_{m+1}$ are precisely the same as the ones for the $\Phi_{i j}^{(0)}$ 's due to $W_{m}$. We conclude that the $\Psi_{i j}^{(0)}$, s give rise to the $\mathrm{CY}_{m+2}$ factor in (11.1).

Let us conclude this section with a few clarifications. The dimensional reduction procedure we have introduced is analogous to, and for $m \leq 2$ coincides with, standard 
dimensional reduction at the level of the classical Lagrangian. We are not making any statement about the well-known subtleties regarding the interplay between dimensional reduction and the renormalization group (RG) flow. In fact, there is no notion of RG flow for $m>2$. In addition, it is clear that, in its current basic form, dimensional reduction does not connect the dualities for $m$ and $m^{\prime}=m+1$. Having said that, we would like to stress that dimensional reduction is, however, a powerful tool for determining quiver theories for CY $(m+3)$-folds starting from those of CY $(m+2)$ folds. This tool will be heavily exploited in the context of the topological B-model in Ref. [18].

\section{CONCLUSIONS AND OUTLOOK}

Let us conclude by summarizing some of the key results of this paper:

(i) In Secs. II-VI, we introduced a mathematical framework based on graded quivers with potentials and higher Ginzburg algebras that provides a unified description of minimally supersymmetric quantum field theories in even dimensions. Moreover, for $m>3$, our framework establishes the existence of an infinite tower of quiver theories with potentials generalizing such gauge theories and specifies their structure. These quivers have a physical implementation in terms of the category of branes in the topological B-model on higher-dimensional CYs. Our mathematical framework is even broader and applies to generic theories regardless of whether or not they have a string theoretic origin.

(ii) In Secs. VII-IX, we discussed in detail the correspondence between the $0 \leq m \leq 3$ cases and gauge theories in six dimensions to zero dimensions. Our framework succinctly captures their field content, interactions, anomaly cancellation, constraints on the Lagrangian coming from SUSY, etc.

Moreover, mutations of these quivers precisely correspond to dualities of the associated quantum field theories. Some of these dualities, like triality and even more so quadrality, have been discovered recently, and their studies are therefore limited. Identifying the mathematical structures underlying them is thus a significant step forward.

(iii) We were led to postulate a completely new, infinite class, of generalizations of the few known dualities to $m>3$. These new mutations encode the transformation of the category of branes of the topological B-model under geometric transitions in the underlying CY $(m+2)$-folds. This problem will be studied in further detail in Ref. [18].

(iv) The mathematical framework has considerable practical applications. In Sec. IV B, we introduced a complete set of local rules for the mutation of the potential for arbitrary $m$. This is particularly useful for cases with a gauge theory interpretation. Let us contextualize this. The $m=1$ case, i.e., $4 \mathrm{D} \mathcal{N}=1$, has been thoroughly studied in the literature. The $m=2$ case, namely, $2 \mathrm{D} \mathcal{N}=(0,2)$, remains relatively less explored. For example, the most detailed studies of the mutation of potentials under triality correspond to toric theories [6]. In this class of models, the dual potential can in principle be determined from periodic quivers. However, there are explicit examples in which this prescription is insufficient, and it needs to be supplemented by additional considerations [6]. The rules introduced in this paper overcome these obstacles. Finally, an algorithmic prescription for the transformation of the potential for $m=3$, i.e., $0 \mathrm{D} \mathcal{N}=1$, was not known. Our results fully solve these issues and, moreover, explicitly answer the question for arbitrary $m$.

(v) In Sec. X, we outlined how to use mirror symmetry to determine the quiver theories associated to toric CY $(m+2)$-folds for $m>3$. We also presented the first example in the literature of a graded quiver with potential associated to a $\mathrm{CY}_{6}$ as well as its construction in terms of mirror symmetry.

(vi) Finally, we introduced a generalization of dimensional reduction to arbitrary $m$. An interesting application of this procedure is the iterative construction of quiver theories for higher-dimensional CY singularities starting from lower-dimensional ones. Infinite classes of such explicit CY/quiver theory pairs, with arbitrarily high $m$, will be presented in Ref. [18].

Our results also considerably extend the mathematical understanding of these theories. In particular:

(i) Our explicit rules for the mutation of potentials supersede the treatment in Ref. [14], which did not incorporate potentials, and are more explicit and combinatorial than the rules in Ref. [15], which are based on differentials for dg algebras. Appendix B explains the nontrivial relation between our approach and the one in Ref. [15].

In addition, the current paper adds an important feature to the mathematical discussion in Ref. [15], namely, the fact that successive mutation at the same node is $(m+1)$ periodic. This is natural from the physics standpoint, see Sec. V, and adds to the mathematics.

Our work suggests several interesting directions for future investigation. A few of them are as follows:

(i) Graded quivers with potentials nicely describe SUSY gauge theories in even dimensions. Does a similar unified description exist for gauge theories and their dualities in odd dimensions?

(ii) In Sec. X, we outlined how higher-dimensional generalizations of dimer models living on $\mathbb{T}^{m+1}$ can be constructed for toric CY $(m+2)$-folds for arbitrary $m$. For $m=2$, such generalizations are called brane brick models and have been introduced in Ref. [5]. Similarly, brane hyperbrick models correspond to $m=3$ and were first postulated in 
Ref. [3]. In future work, we plan to develop these constructions for general values of $m$ and investigate how they bridge geometry to the corresponding quivers. In particular, this will require the generalization of combinatorial notions such as perfect matchings, zigzag paths, etc. [52].

(iii) It would be interesting to extend the mathematical understanding of mutations of graded quivers with potentials to the case in which the mutated node contains adjoint fields. This is a promising direction for uncovering new order- $(m+1)$ dualities for $m>1$. For $m=2$, 3, i.e., $d=2$, 0 , these would be generalizations of triality and quadrality analogous to the generalization of Seiberg duality to 4D SQCD with an adjoint chiral field [54-56].

Furthermore, there are clear questions worth studying in mathematics, which include the following:

(i) The graded quivers, potentials and mutations discussed in this paper match up exactly with oriented flips in $(m+2)$-angulations of polygons. These details will be fleshed out in a forthcoming mathematical companion [57].

(ii) Brane brick models, brane hyperbrick models, and their extensions to $m>3$ lead to higher-dimensional generalizations of perfect matchings [52]. The connection between these objects and higher cluster tilting objects leads to new avenues for investigating algebraic exchange relations that complement graded quiver mutations, thereby extending the algebraic structure beyond the $m=1$ case.

\section{ACKNOWLEDGMENTS}

We would like to thank A. Garver, S. Gurvets, A. Hasan, R.-K. Seong, and especially S. Lee and C. Vafa for enjoyable discussions. We are also indebted to S. Oppermann for useful correspondence. S. F. gratefully acknowledges support from the Simons Center for Geometry and Physics, Stony Brook University, where some of the research for this paper was performed during the Simons Summer Workshop. The work of S. F. is supported by the U.S. National Science Foundation Grant No. PHY-1518967 and by a Professional Staff Congress of the City University of New York (PSCCUNY) award. The work of G. M. is supported by NSF Grant No. DMS-1362980.

\section{APPENDIX A: ALLOWABLE POTENTIAL TERMS AND MUTATIONS}

Definition 1: A configuration of $k$ arrows, with orientations of double arrows chosen so that it is an oriented cycle, graded as $\left(c_{1}, c_{2}, \ldots, c_{k}\right)$ is an allowable potential term if and only if

$$
c_{1}+c_{2}+\cdots+c_{k}=m-1 .
$$

Definition 2: Given a graded quiver $\bar{Q}$, we call a node $i \in Q_{0}$ is mesonic if there is an incoming chiral, i.e., an arrow of degree 0 , incident to $v$. We call that $i$ nonmesonic otherwise.

Claim 4: Any allowable potential term is mutation equivalent to a configuration of the form $(m-1,0,0, \ldots, 0)$ with exactly one nonchiral with degree $(m-1)$ as an oriented cycle via a sequence of nonmesonic mutations. ${ }^{40}$

Proof: A configuration $(m-1,0,0, \ldots, 0)$ clearly satisfies (A1). Furthermore, a nonmesonic mutation replaces a 2-path of arrows having degrees $(d, e)$ with one of degrees $(d-1, e+1)$, and hence the sum on the left-hand side of (A1) is unchanged by such mutations. Thus, it is clear that all allowable potential terms will satisfy identity (A1).

We now show that any $\left(c_{1}, c_{2}, \ldots, c_{k}\right)$ satisfying this equation is indeed reachable from $(m-1,0,0, \ldots, 0)$ via nonmesonic mutations. ${ }^{41}$ First, we note that

$$
\begin{aligned}
\left(c_{1},\right. & \left.c_{2}, \ldots, c_{d}, 0,0, \ldots, 0, c_{k}\right) \\
& \sim\left(c_{1}, c_{2}, \ldots, c_{d}-1,1,0, \ldots, 0, c_{k}\right) \\
& \sim\left(c_{1}, c_{2}, \ldots, c_{d}-1,0,1, \ldots, 0, c_{k}\right) \\
& \sim \cdots \sim\left(c_{1}, c_{2}, \ldots, c_{d}-1,0,0, \ldots, 1, c_{k}\right) \\
& \sim\left(c_{1}, c_{2}, \ldots, c_{d}-1,0,0, \ldots, c_{k}+1\right)
\end{aligned}
$$

by mutating at the $(d+1)$ st, $(d+2)$ nd, $\ldots, k$ th node in order. Using this identity repeatedly, we convert between configurations

$$
\begin{aligned}
& \left(c_{1}, c_{2}, \ldots, c_{d-1}, c_{d}, 0,0, \ldots, 0, c_{k}\right) \\
& \quad \sim\left(c_{1}, 0, \ldots, 0,0,0,0, \ldots, 0, c_{2}+c_{3}+\cdots+c_{d}+c_{k}\right) .
\end{aligned}
$$

Since we assumed that $c_{1}+c_{2}+\cdots+c_{k}=m-1$ up front, this last entry is still from $\{0,1,2, \ldots, m-1\}$. And one final sequence of applications of this identity yields

$$
\begin{aligned}
& \left(0,0, \ldots, 0,0,0,0, \ldots, 0, c_{1}+c_{2}+c_{3}+\cdots+c_{k}\right) \\
& \quad=(0,0, \ldots, 0,0,0,0, \ldots, 0, m-1) .
\end{aligned}
$$

Up to cyclic rotation, we have a configuration of the desired form.

\section{APPENDIX B: MUTATION OF DIFFERENTIALS AND RELATION TO OPPERMANN'S WORK}

We now discuss how the differential structure transforms under mutation. In particular, we will show that $\{W, W\}=$ 0 is preserved by mutations. While doing so, we will

\footnotetext{
${ }^{40}$ This statement applies in the obvious way in the case $m=1$.

${ }^{41}$ In the proof, we assume that no node containing adjoint fields needs to be mutated along the sequence.
} 
discuss the connections between our approach and Oppermann's work in Ref. [15].

Claim 5: If the potential $W$ vanishes under the Kontsevich backet, i.e., $\{W, W\}=0$, then after mutation, the resulting potential $W^{\prime}$ still vanishes under the Kontsevich bracket, i.e., $\left\{W^{\prime}, W^{\prime}\right\}=0$.

Proof: This has been proven as Theorem 8.1 of Ref. [15]. We now provide an alternative proof using the description of differentials given in Sec. III and the mutation rules for the potential in Sec. IV B.
Let us write the potential as

$$
W=\sum_{\sigma \in W} c_{\sigma} W_{\sigma},
$$

where $\sigma$ indicates a cycle in the potential, $W_{\sigma}=$ $\varphi_{1, \sigma} \varphi_{2, \sigma} \cdots \varphi_{k_{\sigma}, \sigma}$ is the corresponding term involving $k_{\sigma}$ arrows, and we explicitly indicate the possibility of numerical coefficients $c_{\sigma}$. For the proof, we set all $c_{\sigma}$ 's to be 1 and introduce a new notation for arrows, in which $\varphi_{i, \sigma}$ indicates the $i$ th arrow in the cycle $\sigma$. Then,

$$
\begin{aligned}
\{W, W\} & =d W=\sum_{\sigma} d\left(W_{\sigma}\right)=\sum_{\sigma} d\left(\varphi_{1, \sigma}\right) \varphi_{2, \sigma} \cdots \varphi_{k_{\sigma}, \sigma}+(-1)^{\left|\varphi_{1, \sigma}\right|} \varphi_{1, \sigma} d\left(\varphi_{2, \sigma} \cdots \varphi_{k_{\sigma}, \sigma}\right) \\
& =\sum_{\sigma}\left(\partial_{\varphi_{1, \sigma}^{\mathrm{op}} W} W \varphi_{2, \sigma} \cdots \varphi_{k_{\sigma}, \sigma}+(-1)^{\left|\varphi_{1, \sigma}\right|} \varphi_{1, \sigma} d\left(\varphi_{2, \sigma}\right) \varphi_{3, \sigma} \cdots \varphi_{k_{\sigma}, \sigma}+(-1)^{\left|\varphi_{1, \sigma}\right|+\left|\varphi_{2, \sigma}\right|} \varphi_{1, \sigma} \varphi_{2, \sigma} d\left(\varphi_{3, \sigma} \cdots \varphi_{k_{\sigma}, \sigma}\right)\right. \\
& =\sum_{\sigma} \sum_{i=1}^{k_{\sigma}}(-1)^{\sum_{j=1}^{i}\left|\varphi_{j, \sigma}\right|} \varphi_{1, \sigma} \varphi_{2, \sigma} \cdots \varphi_{i-1, \sigma}\left(\partial_{\left.\varphi_{i, \sigma}^{\mathrm{op}} W\right) \varphi_{i+1, \sigma} \cdots \varphi_{k_{\sigma}, \sigma} .}\right.
\end{aligned}
$$

In this previous expression, the factors involving derivatives are given by

$$
\partial_{\varphi_{i, \sigma}^{\mathrm{op}}} W=\sum_{\theta \in W, W_{\theta} \supset \varphi_{i, \sigma}^{\mathrm{op}}} \varphi_{1, \theta} \varphi_{2, \theta} \cdots \varphi_{j-1, \theta} \varphi_{j+1, \theta} \cdots \varphi_{k_{\theta}, \theta},
$$

where $\varphi_{i, \sigma}^{\mathrm{op}}$ coincides with $\varphi_{j, \theta}$ and is hence removed from the corresponding summand in (B3). Consequently, the condition $\{W, W\}=0$ in the initial theory implies that the double sum in (B2) decomposes into a sum of alternating sums of the form

$$
\begin{aligned}
& \varphi_{1, \sigma} \varphi_{2, \sigma} \cdots \varphi_{i-1, \sigma} \varphi_{j+1, \theta} \cdots \varphi_{k_{\theta}, \theta} \varphi_{1, \theta} \varphi_{2, \theta} \cdots \varphi_{j-1, \theta} \varphi_{i+1, \sigma} \cdots \varphi_{k_{\sigma}, \sigma} \\
& \quad-(-1)^{\sum_{j=1}^{i}\left|\varphi_{j, \sigma}\right|} \varphi_{j+1, \theta} \cdots \varphi_{k_{\theta}, \theta} \varphi_{1, \theta} \varphi_{2, \theta} \cdots \varphi_{j-1, \theta} \varphi_{i+1, \sigma} \cdots \varphi_{k_{\sigma}, \sigma} \varphi_{1, \sigma} \varphi_{2, \sigma} \cdots \varphi_{i-1, \sigma}
\end{aligned}
$$

The first term comes from the potential terms $W_{\sigma}$ and $W_{\theta}$, while the second one comes from the potential terms

$$
\begin{aligned}
& W_{\beta}=\varphi_{j+1, \theta} \cdots \varphi_{k_{\theta}, \theta} \varphi_{\beta} \varphi_{1, \sigma} \varphi_{2, \sigma} \cdots \varphi_{i-1, \sigma} \\
& W_{\alpha}=\varphi_{i+1, \sigma} \cdots \varphi_{k_{\sigma}, \sigma} \varphi_{\alpha} \varphi_{1, \theta} \varphi_{2, \theta} \cdots \varphi_{j-1, \theta},
\end{aligned}
$$

where $\varphi_{\alpha}^{\mathrm{op}}=\varphi_{\beta}$. Equation (B4) can then be written as

$$
\partial_{\varphi_{i, \sigma}} W_{\sigma} \partial_{\varphi_{j, \theta}} W_{\theta}-\partial_{\varphi_{\alpha}} W_{\alpha} \partial_{\varphi_{\beta}} W_{\beta}
$$

We thus conclude that in $\{W, W\}$ every pair of terms of the form (B6) independently cancels due to signed cyclic equivalence (2.5). Furthermore, for each cancellation, it is only necessary to focus on a set of four potential terms of the type $W_{\sigma}+W_{\theta}+W_{\alpha}+W_{\beta}$. Figure 31 shows a graphical representation of this process. This provides a clear strategy for proving that $\left\{W^{\prime}, W^{\prime}\right\}=0$ after mutation: it is sufficient to follow the evolution of such combinations of four potential terms. There are two possibilities, depending on whether some of the arrows in (B6) pass through the mutated node $k$ or not. Below, we analyze each of them independently.

Let us first consider the case in which no arrow in (B6) runs through node $k$. Then, mutating at node $k$ will leave $W_{\sigma}+W_{\theta}+W_{\alpha}+W_{\beta}$ and the alternating sum of (B4) invariant, and it will thus still vanish after mutation.

Next, let us consider the case in which some arrows in (B6) go through node $k$. This case can be separated into two possibilities. First, let us assume that node $k$ is not incident to $\varphi_{i, \sigma}^{\mathrm{op}}=\varphi_{j, \theta}$ nor $\varphi_{\alpha}^{\mathrm{op}}=\varphi_{\beta}$. Instead, node $k$ is at some intermediate point of the 2-path $\stackrel{\varphi_{r, \sigma}^{\left(r_{r}\right)}}{\longrightarrow} \stackrel{\varphi_{r+1, \sigma}^{\left(c_{r+1}\right)}}{\longrightarrow} \cdot$, where we have expanded our notation to indicate the degree of the arrows in the exponents. If the degree $c_{r}=0$, i.e., if $\varphi_{r, \sigma}^{(0)}$ is a chiral going into $k$, then a mesonic arrow corresponding to the composition $\varphi_{r, \sigma} \varphi_{r+1, \sigma}$ is created according to rule 2. Following rule 2.b, the product $\varphi_{r, \sigma} \varphi_{r+1, \sigma}$ is replaced by the meson in both $W_{\sigma}$ and $W_{\alpha}$ (respectively, $W_{\beta}$ ). The resulting alternating sum in $\left\{W^{\prime}, W^{\prime}\right\}$ still vanishes. Additionally, a new cubic term is added to the potential 

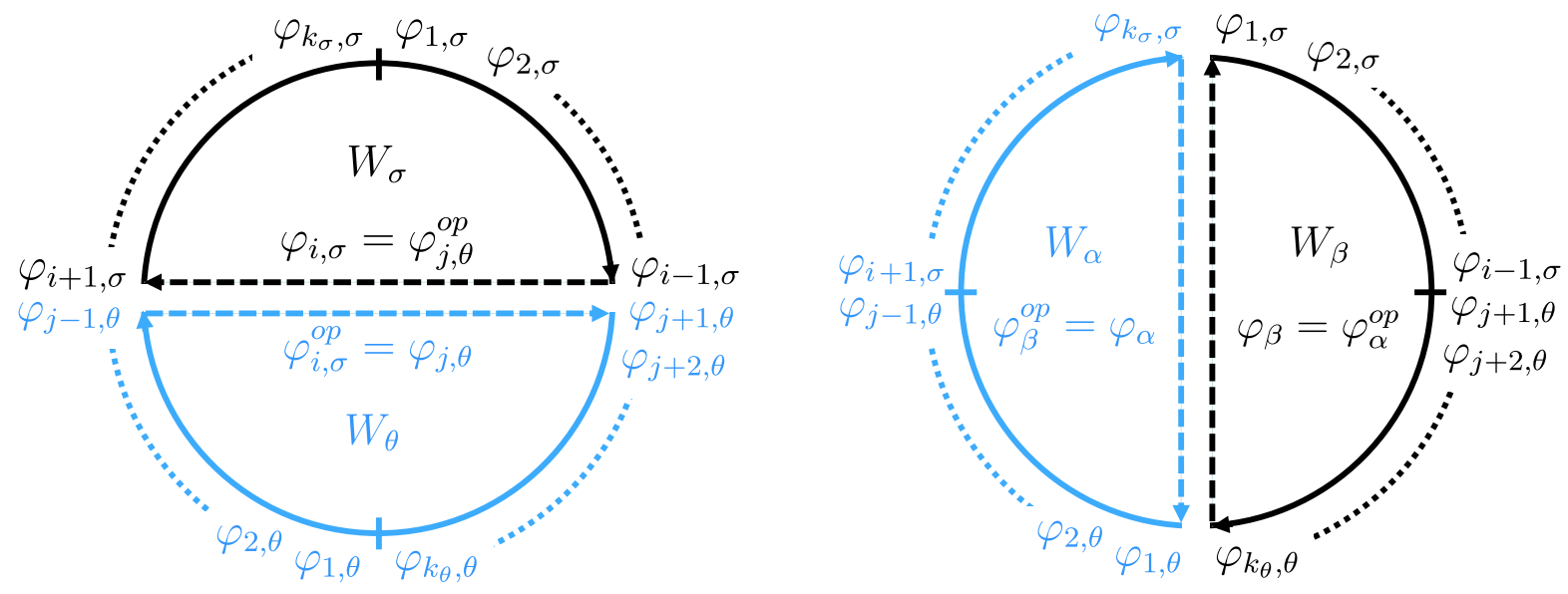

FIG. 31. Graphical representation of the two terms in (B4), which follow from four potential terms $W_{\sigma}+W_{\theta}+W_{\alpha}+W_{\beta}$.
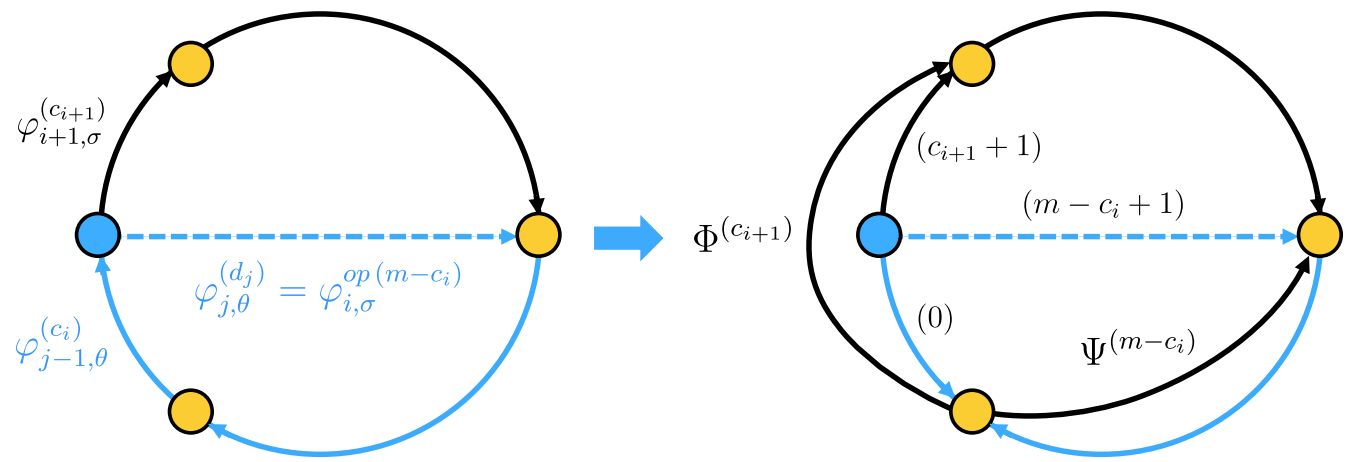

FIG. 32. Mutation at a node sitting at a triple intersection of arrows in Fig. 31. In this figure, we assume $c_{i}=0$ and $d_{j}=m-c_{i}$.

according to rule 2.a, but since the opposite of this mesonic arrow is not also adjoined, this contributes 0 to $\left\{W^{\prime}, W^{\prime}\right\}$ as well. If instead $c_{r} \neq 0$, then we use rule 2.c instead and replace $\varphi_{r, \sigma}^{\left(c_{r}\right)} \varphi_{r+1, \sigma}^{\left(c_{r+1}\right)}$ with $\varphi_{r, \sigma}^{\left(c_{r}-1\right)} \varphi_{r+1, \sigma}^{\left(c_{r+1}+1\right)}$. Again, after this replacement, $\left\{W^{\prime}, W^{\prime}\right\}=0$ still holds.

Lastly, let us consider the other possibility, in which we mutate at $k$, which is incident to $\varphi_{j, \theta}$ or $\varphi_{\beta}$. This means that the mutated node is located at the end point of one of the dashed arrows in Fig. 31, at the triple intersection between a dashed arrow and the two solid lines of a different color. ${ }^{42}$ We illustrate this situation in Fig. 32, in which we explicitly indicate the mutated node (blue) and the three other relevant nodes connected to it (yellow).

Without loss of generality, we consider the node at the intersection of the three arrows $\varphi_{j-1, \theta}$ (incoming), $\varphi_{j, \theta}=$ $\varphi_{i, \sigma}^{\mathrm{op}}$ (outgoing), and $\varphi_{i+1, \sigma}$ (outgoing). We then apply rules 2.a-2.d as appropriate, depending on whether $\varphi_{j-1, \theta}$ is

\footnotetext{
${ }^{42}$ We assume such cycles go through node $k$ only once. The proof for cycles that pass multiple times through the mutated node is analogous but requires a more lengthy analysis.
}

chiral or not. We replace $W_{\sigma}+W_{\theta}+W_{\alpha}+W_{\beta}$ with $W_{\sigma}^{\prime}+W_{\theta}^{\prime}+W_{\alpha}^{\prime}+W_{\beta}^{\prime}+W_{D}^{\prime}$, where $W_{D}^{\prime}$ is the new term arising from rule 2 .d. We obtain

$$
\begin{aligned}
W_{\sigma}^{\prime}= & \varphi_{i+1, \sigma}^{\left(c_{i+1}+1\right)} \varphi_{i+2, \sigma}^{\left(c_{i+2}\right)} \cdots \varphi_{i-1, \sigma}^{\left(c_{i-1}\right)} \overline{\varphi_{j, \theta}}\left(c_{i}-1\right) \\
W_{\theta}^{\prime}= & \varphi_{j+1, \theta}^{\left(d_{j+1}\right)} \varphi_{j+2, \theta}^{\left(d_{j+2}\right)} \cdots \varphi_{j-2, \theta}^{\left(d_{j-2}\right)} \Psi^{\left(m-c_{i}\right)} \\
& +\Psi^{\left(m-c_{i}\right)} \overline{\bar{\varphi}_{j, \theta}}\left(c_{i}-1\right) \overline{\varphi_{j-1, \theta}}(0) \\
W_{\alpha}^{\prime}= & \varphi_{1, \theta}^{\left(d_{1}\right)} \varphi_{2, \theta}^{\left(d_{2}\right)} \cdots \varphi_{j-2, \theta}^{\left(d_{j-2}\right)} \Phi^{\left(c_{i+1}\right)} \varphi_{i+2, \sigma}^{\left(c_{i+2}\right)} \cdots \varphi_{k_{\sigma}, \sigma}^{\left(c_{k_{\sigma}}\right)} \varphi_{\alpha}^{(a)} \\
& +\Phi^{\left(c_{i+1}\right)}{\overline{\varphi_{i+1, \sigma}}}^{\left(m-c_{i+1}-1\right)} \bar{\varphi}_{j-1, \theta}^{(0)} \\
W_{\beta}^{\prime}= & W_{\beta}=\varphi_{j+1, \theta}^{\left(d_{j+1}\right)} \cdots \varphi_{k_{\theta},}^{\left(d_{k_{\theta}}\right)} \bar{\varphi}_{\alpha}^{(m-a)} \varphi_{1, \sigma}^{\left(c_{1}\right)} \varphi_{2, \sigma}^{\left(c_{2}\right)} \cdots \varphi_{i-1, \sigma}^{\left(c_{i-1}\right)} \\
W_{D}^{\prime}= & -\bar{\Psi}^{\left(c_{i}\right)} \Phi^{\left(c_{i+1}\right)} \varphi_{i+2, \sigma}^{\left(c_{i+2}\right)} \cdots \varphi_{i-1, \sigma}^{\left(c_{i-1}\right)},
\end{aligned}
$$

where, in order not to clutter the notation, we used $\bar{\varphi}$ for $\varphi^{\mathrm{op}}$. Keeping track of the extra negative term resulting from rule 2.d, we indeed obtain $\left\{W^{\prime}, W^{\prime}\right\}=0$ in this case as well. In particular, $\left\{W_{\sigma}^{\prime}+W_{\theta}^{\prime}+W_{\alpha}^{\prime}+W_{\beta}^{\prime}+W_{D}^{\prime}, W_{\sigma}^{\prime}+\right.$ $\left.W_{\theta}^{\prime}+W_{\alpha}^{\prime}+W_{\beta}^{\prime}+W_{D}^{\prime}\right\}$ equals 


$$
\begin{aligned}
\partial_{\overline{\Psi^{\left(c_{i}\right)}}} & W_{D^{\prime}} \partial_{\Psi^{\left(m-c_{i}\right)}} W_{\theta^{\prime}}+\partial_{\varphi_{\alpha}^{(a)}} W_{\alpha^{\prime}} \partial_{\bar{\varphi}_{\alpha}(m-a)} W_{\beta^{\prime}} \\
& +\partial_{\varphi_{i+1, \sigma}^{\left(c_{i+1}+1\right)}} W_{\sigma^{\prime}} \partial_{\overline{\varphi_{i+1, \sigma}}}^{\left(m-c_{i+1}-1\right)} W_{\alpha^{\prime}} \\
= & -\Phi^{\left(c_{i+1}\right)} \varphi_{i+2, \sigma}^{\left(c_{i+2}\right)} \cdots \varphi_{i-1, \sigma}^{\left(c_{i-1}\right)} \varphi_{j+1, \theta}^{\left(d_{j+1}\right)} \varphi_{j+2, \theta}^{\left(d_{j+2}\right)} \cdots \varphi_{j-2, \theta}^{\left(d_{j-2}\right)} \\
& -\Phi^{\left(c_{i+1}\right)} \varphi_{i+2, \sigma}^{\left(c_{i+2}\right)} \cdots \varphi_{i-1, \sigma}^{\left(c_{i-1}\right)} \overline{\varphi_{j, \theta}}\left(c_{i}-1\right) \overline{\varphi_{j-1, \theta}} \\
& +\varphi_{1, \theta}^{\left(d_{1}\right)} \varphi_{2, \theta}^{\left(d_{2}\right)} \cdots \varphi_{j-2, \theta}^{\left(d_{j-2}\right)} \Phi^{\left(c_{i+1}\right)} \varphi_{i+2, \sigma}^{\left(c_{i+2}\right)} \cdots \varphi_{k_{\sigma}, \sigma}^{\left(c_{k_{\sigma}}\right)} \varphi_{1, \sigma}^{\left(c_{1}\right)} \varphi_{2, \sigma}^{\left(c_{2}\right)} \cdots \\
& \times \varphi_{i-1, \sigma}^{\left(c_{i-1}\right)} \varphi_{j+1, \theta}^{\left(d_{j+1}\right)} \cdots \varphi_{k_{\theta}, \theta}^{\left(d_{k_{\theta}}\right)} \\
& +\varphi_{i+2, \sigma}^{\left(c_{i+2}\right)} \cdots \varphi_{i-1, \sigma}^{\left(c_{i-1}\right)}{\overline{\varphi_{j, \theta}}}^{\left(c_{i}-1\right)} \bar{\varphi}_{j-1, \theta}{ }^{(0)} \Phi^{\left(c_{i+1}\right)}=0
\end{aligned}
$$

up to signed cyclic equivalence.

The prescription for mutating the potential and reducing it via massive terms that we introduced in Sec. IV B was partially motivated by Sec. VI of Oppermann's work [15], which is formulated in terms of the higher Ginzburg algebra. The latter has the advantage of showing that the derived endomorphism ring of the graded quiver with potential is invariant under mutation (Ref. [15], Theorem 1.1). In the remainder of this section, we discuss how the terminology of Ref. [15] compares with ours.

To help the reader interested in a more detailed comparison with Ref. [15], we will clarify the notation in that paper and use it in the discussion that follows. There are two new operations acting on arrows $\alpha$ of the quiver:

(i) $\alpha^{-1}$.- Roughly speaking, this is the same as $\alpha_{\mathrm{op}}$. The distinction between the two is subtle and depends on whether we regard the quiver as consisting of single arrows, in which case we use $\alpha^{-1}$ to label a piece of a mesonic arrow, or double arrows, for which we use the notation $\alpha_{\mathrm{op}}$.

(ii) $\alpha^{*}$. - This is a compact way of indicating a mutated flavor, namely, the mutation of an arrow connected to the mutated node. The rule for mutating flavors was given in Sec. IV A. Oppermann's convention is to also flip the orientation of the flavors. Hence, in his notation, our mutation takes the form $\alpha \rightarrow \alpha_{\mathrm{op}}^{*}$.

In Ref. [15], the transformation of the potential in a mutation on node $k$ is described in an extremely compact form as

$$
W \rightarrow W^{\prime}=\operatorname{dec}_{\mathrm{cyc}} W+\sum_{\substack{\alpha:\left(\frac{0)}{)} k \\ \varphi: k \stackrel{(c)}{\longrightarrow}\right.}} \alpha \operatorname{dec}(\varphi \bar{\varphi}) \alpha^{*}
$$

Such a compact expression becomes possible thanks to the introduction of the functions dec and $\mathrm{dec}_{\mathrm{cyc}}$, defined as an action on a cycle $\gamma$ and then extended linearly to act on a potential. In particular, if $\gamma$ is a cycle that is never incident to node $k$, the node in which mutation is occurring, then $\operatorname{dec}(\gamma)$ and $\operatorname{dec}_{\mathrm{cyc}}(\gamma)$ are defined to be equal to $\gamma$.
Otherwise, for every 2-path $\varphi_{i, k} \varphi_{k, j}$ contained $^{43}$ inside of $\gamma$, we replace such a 2-path with the element $\left(\varphi_{i, k} \varphi_{k, j}-\sum_{\alpha: \underset{(0)}{\rightarrow} k} \varphi_{i, k} \alpha^{-1} \alpha \varphi_{k, j}\right)$. The result is $\operatorname{dec}(\gamma)$. For the case of $\operatorname{dec}_{\text {cyc }}(\gamma)$, this operation is taken cyclically, meaning that if $\gamma$ starts and ends at the node $k$, then $\left(1-\sum_{\alpha: \underset{(0)}{\rightarrow} k} \alpha^{-1} \alpha\right)$ is also multiplied at the beginning of the cycle. In particular, to compare our combinatorial rule for mutation of potentials to that in Ref. [15], we split his rule into two pieces: (a) $W \rightarrow \operatorname{dec}_{\text {cyc }} W$

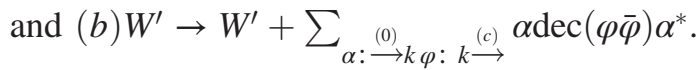

The first piece agrees with our rules 2.b and 2.c implicitly, since terms appearing in the potential remain in it after mutation. The only differences are that the degrees of the arrows are updated accordingly and, in the case of a composition or anticomposition involving a degree-0 incoming arrow $\alpha$, the 2-path $\alpha \varphi$ (respectively, $\varphi \alpha^{-1}$ ) becomes a single arrow $[\alpha \varphi]$ (respectively, $\left[\varphi \alpha^{-1}\right]$ ) of degree matching that of $\varphi$ prior to mutation. The square bracket notation $[\alpha \beta]$ indicates this single mesonic arrow corresponding to the composition of $\alpha$ and $\beta$.

On the other hand, rule 2.d is applied explicitly since the insertion of the factor $\left(1-\sum_{\alpha: \underset{(0)}{\rightarrow} k} \alpha^{-1} \alpha\right)$ yields ${ }^{44}$ one new potential term in which the $\alpha$ and $\alpha^{-1}$ are paired with two different flavors to yield new mesonic arrows $\left[\varphi_{i k} \alpha^{-1}\right]$ and $\left[\alpha \varphi_{k j}\right]$. Focusing on the prescence of a single chiral from a vertex denoted as $i_{0}$, i.e., denoted as $\alpha: i_{0} \rightarrow^{(0)} k$, we have $\left[\varphi_{i k} \alpha^{-1}\right]: i \rightarrow^{(c)} i_{0}$ and $\left[\alpha \varphi_{k j}\right]: i_{0} \rightarrow^{(d)} j$. This is consistent since this insertion of the above factor turns the term $W_{\gamma}=\varphi_{i_{1}, i_{2}} \cdots \varphi_{i_{r}, i} \varphi_{i, k} \varphi_{k, j} \varphi_{j, i_{r+4}} \cdots \varphi_{i_{\ell}, i_{1}}$ into $W_{\gamma}-\varphi_{i_{1}, i_{2}} \cdots$ $\varphi_{i_{r}, i}\left[\varphi_{i, k} \alpha^{-1}\right]\left[\alpha \varphi_{k, j}\right] \varphi_{j, i_{r+4}} \cdots \varphi_{i_{\ell}, i_{1}}$.

Lastly, rule 2.a, which adjoins a new cubic term involving a product of three arrows for every composition $[\alpha \varphi]$, involving an incoming arrow $\alpha$ of degree 0 and any outgoing arrow $\varphi$, is exactly of the form ${ }^{45}$

$$
\alpha \operatorname{dec}(\varphi \bar{\varphi}) \alpha^{*}=[\alpha \varphi] \bar{\varphi} \alpha^{*} .
$$

Notice that, since we are assuming that there are not adjoints at the node under mutation, we indeed have $\operatorname{dec}(\varphi \bar{\varphi})=\varphi \bar{\varphi}$. In particular, the source of $\bar{\varphi}$ is a node other than $k$.

\footnotetext{
${ }^{43}$ For convenience, we now think of cycle $\gamma=\varphi_{i_{1}, i_{2}} \varphi_{i_{2}, i_{3}} \ldots$ $\varphi_{i_{\ell}, i_{1}}$, where the subscripts denote the head and tail of the constituent arrows. We also let $i, k$, and $j$ label three of these vertices in a row.

${ }^{44}$ Originally, our rule $2 . d$ did not specify the sign of the new potential term. However, as motivated by Ref. [15] and to ensure $\left\{W^{\prime}, W^{\prime}\right\}=0$, it is mathematically natural to give such new potential terms from the case of two mesons a coefficient of -1 .

${ }^{45}$ In Ref. [15], potential terms are read right to left rather than left to right, and outgoing chirals are used instead of incoming chirals. These two reversals from our convention cancel each other out.
} 
Note that Secs. 7 and 8 of Ref. [15] explicitly describe the cyclic derivatives with respect to certain arrows. Putting this together, the vanishing of the square of the differential in the mutated theory is proved. This is equivalent to showing that the Kontsevich bracket vanishes for the mutated potential, hence giving an alternative to our proof based on the mutation rules given in Sec. IV B.

\section{APPENDIX C: BACKGROUND ON CLUSTER CATEGORIES}

We include a brief background on cluster categories and higher cluster categories for the interested reader. This provides some of the motivation behind graded (i.e., colored) quivers from the mathematical perspective.

We first focus on the $m=1$ case of ordinary quivers. The path algebra $k Q$ of a finite acyclic quiver $Q$, i.e., a quiver that contains no cycles, satisfies a lot of important mathematical properties. Such a path algebra is a hereditary finite-dimensional basic algebra, and its modules form an Abelian $k$ category that is also Krull-Schmidt. In other words, given two representations (equivalently, modules) of the path algebra $M$ and $N$, the set of homomorphisms between $M$ and $N$ is a $k$-vector space denoted as $\operatorname{Hom}(M, N)$. Further, any representation can be written as a direct sum of indecomposable representations in a unique way up to reordering.

From this, we form $\mathcal{D}(k Q)$, the bounded derived category of $k Q$ with shift functor [1], the indecomposable objects of which are all of the form $M[i]$, where $M$ is an indecomposable of $k Q$ and $i \in \mathbb{Z}$ signifies an application the shift functor (or its inverse) a certain number of times. The bounded derived category is a triangulated category, which means that we can write down certain sequences $A \rightarrow B \rightarrow C \rightarrow$ known as (distinguished) triangles.

Given an indecomposable representation $C$, there is a unique triangle $\rightarrow_{-} \rightarrow C \rightarrow$. We define the AuslanderReiten translation of indecomposable $C$ to be $\tau C=A$, unique indecomposable that fills in to the leftmost object of the distinguished triangle with $C$ as the rightmost object. The Auslander-Reiten translation has the property that it sends projective indecomposable objects to 0 and otherwise sends nonprojective indecomposables to indecomposables.

We work with a certain quotient of the bounded derived category known as the cluster category $\mathcal{C}_{1}(H)$ defined as $\mathcal{D}(H) /\left(\tau^{-1} \circ[1]\right)$, where $\tau$ is Auslander-Reiten translation and [1] is the shift functor. Because of this identification, if $P_{i}$ is the projective indecomposable associated to node $i \in Q_{0}$, then $\tau P_{i}=P_{i}[1]$ rather than zero in $\mathcal{C}_{1}(H)$. Furthermore, $\tau P_{i}[1]=I_{i}$, the injective indecomposable associated to node $i \in Q_{0}$.

Remark 2: For a finite acyclic quiver $Q$ and a node $i \in Q_{0}$, the projective indecomposable $P_{i}$ is the module with the basis given by all paths beginning at $i$ and ending at any other node. In particular, $P_{i}=k Q e_{i}$. In contrast, the injective indecomposable $I_{i}$ is the module with the basis given by all paths ending at $i$, i.e., $I_{i}=e_{i} k Q$. Lastly, $k Q=$ $P_{1} \oplus P_{2} \oplus \cdots \oplus P_{n}$ as a module because of the orthogonality of idempotents.

The cluster category is again triangulated and KrullSchmidt and also has the property that it is a 2-Calabi-Yau category, meaning that the Serre functor $\nu=[1] \tau$ is equivalent to [2] (since $\tau^{-1} \circ[1] \sim i d$ ). Cluster categories provide a categorification for cluster algebras, and by the CalderoChapoton map, the Laurent expansions of cluster variables even correspond to rigid indecomposables of $\mathcal{C}_{1}(H)$.

This motivated the higher m-cluster category, which is the triangulated $(m+1)$-Calabi-Yau category obtained by the quotient $\mathcal{D}(H) /\left(\tau^{-1} \circ[m]\right)$. Here, $(m+1)$-Calabi-Yau signifies that the Serre function $\nu=[1] \tau \sim[m+1]$.

The cluster-tiling objects are maximally dimensional direct sums of indecomposables that have no self-extensions. They can also be organized into what are called exchange triangles,

$$
\begin{aligned}
X_{i} & \rightarrow B_{0} \rightarrow X_{i}^{\prime} \rightarrow \\
X_{i}^{\prime} & \rightarrow B_{1} \rightarrow X_{i}^{\prime \prime} \rightarrow \\
X_{i}^{\prime \prime} & \rightarrow B_{2} \rightarrow X_{i}^{\prime \prime \prime} \rightarrow \\
& \vdots \\
X_{i}^{(m)} & \rightarrow B_{m} \rightarrow X_{i} \rightarrow,
\end{aligned}
$$

where

$$
B_{c}=\bigoplus_{\substack{(c) \\ \varphi: i \stackrel{\rightarrow}{\rightarrow} j}} X_{j}
$$

Higher tilting objects are $X_{1} \oplus X_{2} \oplus \ldots \oplus X_{n}$, where we take one element out, i.e., $X_{i}$, and then $B_{0}$ is some direct sum of the other $X_{j}$ 's. These category theory definitions generalizing tilting theory (from the 1970s) lead to the work of Ref. [14] to define graded quivers combinatorially from this algebra. See Ref. [58] for a related but different treatment.

From a physical point of view, these exchange triangles also give rise to a relationship between ranks

$$
N_{B_{0}}=N_{X_{i}}+N_{X_{i}^{\prime}},
$$

which nicely agrees with the transformation rule for ranks under mutation (4.1).

Note that in the $m=1$ case this sequence reduces to

$$
X_{i} \rightarrow B_{0} \rightarrow X_{i}^{\prime} \rightarrow \quad \text { and } \quad X_{i}^{\prime} \rightarrow B_{1} \rightarrow X_{i} \rightarrow
$$

because of the $(m+1)=2$ periodicity. In fact, this leads to a single exchange relation,

$$
C C\left(X_{i}\right) C C\left(X_{i}^{\prime}\right)=C C\left(B_{0}\right)+C C\left(B_{1}\right),
$$




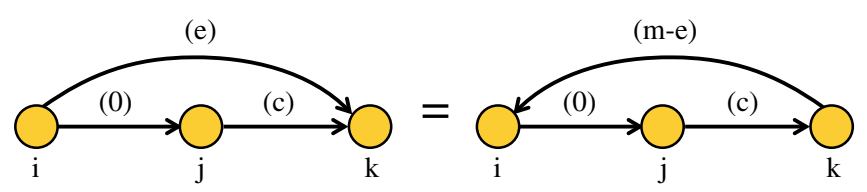

FIG. 33. A special local configuration considered in Ref. [14].

where $C C$ denotes the Caldero-Chapton map $[59,60]$. This relates the cluster category to cluster variables and cluster algebras in the $m=1$ case. An analog of such an algebraic structure for higher $m$ is an open question.

\section{Constraints in the potential and higher cluster categories}

The treatment of graded quivers and higher cluster categories in Ref. [14] does not include potentials. ${ }^{46}$ However, that work proposes a condition that is closely related to our general discussion of potentials. Proposition 5.1 of Ref. [14] states that if a graded quiver associated to a higher-cluster tilting object has a local configuration of the form shown in Fig. 33 then the degree $(e)$ must be $(c)$ or $(c+1)$. This local configuration is an allowable potential term if and only if $(e)=(c+1)$.

Forbidding other cyclic configurations of three arrows is important because otherwise mutation at the middle node $j$ would lead to a configuration in which monochromaticity breaks. In particular, as we saw in Sec. II C 2, the only massive potential terms are of the form $\varphi_{i k}^{(c)} \varphi_{k i}^{(m-c-1)} \sim \varphi_{i k}^{(c)} \varphi_{i k}^{(c+1)}$. As a result, only 2-cycles arising from mutation at the middle term where Proposition 5.1 of Ref. [14] holds, i.e., $e=c+1$, can be deleted. The other case of $(e)=(c)$ yields multiple arrows of degree $(c)$.

\section{Comments on mesons at nodes with multiple incoming chirals}

Notice that rule 2 of Sec. IVA regarding the generation of mesons under mutation forbids new mesons coming from 2-paths of the form $i \stackrel{(0)}{\rightarrow} \stackrel{(m)}{\rightarrow} k$, or equivalently from the anticomposition $i \stackrel{(0)}{\rightarrow} j \stackrel{(0)}{\leftarrow} k$. This differs from Buan and Thomas (Ref. [14], Sec. X), which creates new arrows from such compositions. In fact, they not only generate a new arrow $\left.i \frac{(m)}{j}\right)_{k}$ from the composition $i \stackrel{(0)}{\rightarrow} \stackrel{(m)}{\rightarrow} k$ but also a new arrow $i \frac{j}{(m)} k$ (which is equivalent to $i \underset{j}{j} k$ ). This second arrow

\footnotetext{
${ }^{46}$ While that work does not consider potentials explicitly, it is possible to argue that their manipulations are mostly consistent with assuming a totally generic potential. This statement is true, modulo the observation we made in Sec. IV B regarding the inconsistent removal of chiral-chiral pairs for $m>1$, for which they cannot form mass terms in the potential, in Ref. [14].
}

arises since every arrow in sight is in reality a double arrow and thus the configuration $i \stackrel{(0)}{\rightarrow} j \stackrel{(m)}{\rightarrow} k$ is not only equivalent to $i \stackrel{(0)}{\rightarrow} j \stackrel{(0)}{\leftarrow} k$ but is also equivalent to $i \stackrel{(m)}{\longleftarrow} j \stackrel{(0)}{\longleftarrow} k$, and so we have compositions in both directions. The authors of Ref. [14] include a rule that removes any chiral-chiral 2cycle, which results in the cancellation of these two arrows between nodes $i$ and $k$. Since they do not work with potentials for graded quivers, the result of constructing two new mesons from such compositions and then canceling them as a pair is identical to our rule of this paper forbidding compositions from $i \stackrel{(0)}{\rightarrow} j \stackrel{(c)}{\rightarrow} k$ with $c=m$ in the first place.

Not only does rule 2.a as we stated it avoid extra bookkeeping that would arise from the creation and deletion of such 2-cycles, but more importantly, it is in fact necessary since, as explained in Sec. II C 2, chiralchiral pairs cannot correspond to mass terms in the potential for $m>1$. Hence, this 2-cycle could not correspond to a mass term, and rule 3 would not apply for reducing it. Nonetheless, up to this nuance, ${ }^{47}$ the definition of colored quiver mutation in Ref. [14] indeed agrees with our definition of graded quiver mutation. Furthermore, while they do not implement the full treatment that would follow from a potential, the results they focus on are insensitive to this discrepancy.

\section{APPENDIX D: SILTING}

There is a variant of tilting, known as silting. Roughly speaking, it can be regarded as the $m \rightarrow \infty$ or $\mathrm{CY}_{\infty}$ limit of graded quivers and their dualities. In particular, in this case, there is no upper limit on the degree of arrows, which can grow arbitrarily under mutations. Similarly, sequences of repeated mutations on the same node are not periodic. Oppermann's work on potentials for graded quivers [15] was in fact motivated by considering this particular setting. In this Appendix, we sketch the relationship between silting and higher cluster categories, thereby providing the mathematical bridge between Buan and Thomas [14] and Oppermann [15].

Following Buan et al. [61], we now compare silting objects and $m$-cluster tilting objects. Let $H$ be a finitedimensional hereditary algebra. For $m \geq 1$, the $m$-cluster category $\mathcal{C}_{m}$ is defined as the quotient category $\mathcal{D} / \tau^{-1}[m]$. Here, $\mathcal{D}$ is the bounded derived category of $H, \tau$ is Auslander-Reiten translation in $\mathcal{D}$, and $[m]$ signifies applying the shift functor $[1] m$ times.

The $m$-cluster category is a Krull-Schmidt category, meaning that we can decompose objects into finite direct sums of indecomposables. Let $\bmod H$ denote the indecomposables objects of $H$ and $\bmod H[i]$ denote the set after

\footnotetext{
${ }^{47}$ As well as a reversal of the role of incoming and outgoing that we include to better match physics.
} 
applying $i$ copies of the shift functor to each indecomposable. Because $\mathcal{C}_{m}$ is a quotient of $\mathcal{D}$, a fundamental domain for the set of indecomposables is given by

$$
\begin{aligned}
\mathcal{S}_{m}= & \bmod H[0] \cup \bmod H[1] \cup \ldots \cup \bmod H[m-1] \\
& \times \cup\left\{P_{1}[m], P_{2}[m], \ldots, P_{n}[m]\right\},
\end{aligned}
$$

where $P_{1}, P_{2}, \ldots, P_{n}$ are projective objects in $H$.

Proposition 1: (Proposition 2.4 of Ref. [61]): With the above setup, let $T$ be an object of $\mathcal{D}$ given as a direct sum of indecomposables, all of which lie in $\mathcal{S}_{m}$. Then, $T$ is a silting object if and only if $T$ is an $m$-cluster tilting object in $\mathcal{C}_{m}$.

Theorem 1: (Theorem 3.5, Corollary 3.6 of Ref. [61]): Let $T_{1} \oplus T_{2} \oplus \cdots T_{n}$ be a basic silting object in $\mathcal{D}$, where $T_{i}$ are indecomposable and $n$ is the number of isomorphism classes of simple $H$ modules (here, "basic" means that each of the $T_{i}$ 's are distinct). Assume that all $T_{i}=M[j]$, where $M$ is an indecomposable of $H$ and $j \geq 0$. Choose $m$ large enough so that each $T_{i} \in \mathcal{S}_{m}$.

Then, for each $i \in\{1,2, \ldots, n\}$, there are $(m+1)$ nonisomorphic complements $M_{0}, M_{1}, \ldots, M_{m}$ lying in $\mathcal{S}_{m}$ for the almost complete silting object

$$
T / T_{i}=T_{1} \oplus T_{2} \oplus \cdots \oplus T_{i-1} \oplus T_{i+1} \oplus \cdots \oplus T_{n}
$$

Furthermore, $T / T_{i}$ has a countably infinite number of nonisomorphic complements $M_{i}$ for $i \in \mathbb{Z}$, where there exists $M_{-1}$ and $M_{m+1}$ such that $M_{j+m+1}=M_{m+1}[j]$ and $M_{-j-1} \cong M_{-1}[-j]$ for $j \geq 0$.
[1] N. Seiberg, Electric-magnetic duality in supersymmetric nonAbelian gauge theories, Nucl. Phys. B435, 129 (1995).

[2] A. Gadde, S. Gukov, and P. Putrov, $(0,2)$ trialities, J. High Energy Phys. 03 (2014) 076.

[3] S. Franco, S. Lee, R.-K. Seong, and C. Vafa, Quadrality for supersymmetric matrix models, J. High Energy Phys. 07 (2017) 053.

[4] S. Franco, D. Ghim, S. Lee, R.-K. Seong, and D. Yokoyama, $2 d(0,2)$ quiver gauge theories and D-branes, J. High Energy Phys. 09 (2015) 072.

[5] S. Franco, S. Lee, and R.-K. Seong, Brane brick models, toric Calabi-Yau 4-folds and 2d $(0,2)$ quivers, J. High Energy Phys. 02 (2016) 047.

[6] S. Franco, S. Lee, and R.-K. Seong, Brane brick models and 2d (0, 2) triality, J. High Energy Phys. 05 (2016) 020.

[7] S. Franco, S. Lee, and R.-K. Seong, Orbifold reduction and 2d (0,2) gauge theories, J. High Energy Phys. 03 (2017) 016.

[8] S. Franco, D. Ghim, S. Lee, and R.-K. Seong, Elliptic genera of $2 \mathrm{~d}(0,2)$ gauge theories from brane brick models, J. High Energy Phys. 06 (2017) 068.

[9] S. Franco, S. Lee, R.-K. Seong, and C. Vafa, Brane brick models in the mirror, J. High Energy Phys. 02 (2017) 106.

[10] R. Tatar, Geometric constructions of two dimensional $(0,2)$ SUSY theories, Phys. Rev. D 92, 045006 (2015).

[11] F. Benini, N. Bobev, and P. M. Crichigno, Two-dimensional SCFTs from D3-branes, J. High Energy Phys. 07 (2016) 020.

[12] S. Schafer-Nameki and T. Weigand, F-theory and 2d $(0,2)$ theories, J. High Energy Phys. 05 (2016) 059.

[13] F. Apruzzi, F. Hassler, J. J. Heckman, and I. V. Melnikov, UV completions for non-critical strings, J. High Energy Phys. 07 (2016) 045.

[14] A. B. Buan and H. Thomas, Coloured quiver mutation for higher cluster categories, Adv. Math. 222, 971 (2009).

[15] S. Oppermann, Quivers for silting mutation, Adv. Math. 307, 684 (2017).
[16] M. Van den Bergh, Calabi-Yau algebras and superpotentials, Selecta Math. (N.S.) 21, 555 (2015).

[17] F. Cachazo, B. Fiol, K. A. Intriligator, S. Katz, and C. Vafa, A geometric unification of dualities, Nucl. Phys. B628, 3 (2002).

[18] B-Branes, Calabi-Yau singularities and graded quivers (to be published).

[19] R. Bocklandt and L. Le Bruyn, Necklace Lie algebras and noncommutative symplectic geometry, Math. Z. 240, 141 (2002).

[20] V. Ginzburg, Non-commutative symplectic geometry, quiver varieties, and operads, Math. Res. Lett. 8, 377 (2001).

[21] M. Kontsevich, Formal (non)commutative symplectic geometry, in The Gelfand Mathematical Seminars, 19901992 (Birkhäuser, Boston, MA, 1993), p. 173.

[22] V. Ginzburg, Calabi-Yau algebras, arXiv:math/0612139.

[23] B. Keller and D. Yang, Derived equivalences from mutations of quivers with potential, Adv. Math. 226, 2118 (2011).

[24] S. Ladkani, Finite-dimensional algebras are $(m>2)$ Calabi-Yau tilted, arXiv:1603.09709.

[25] M. A. Bertani-Økland and S. Oppermann, Mutating loops and 2-cycles in 2-CY triangulated categories, J. Algebra 334, 195 (2011).

[26] S. Fomin and A. Zelevinsky, Cluster algebras. I. Foundations, J. Am. Math. Soc. 15, 497 (2002).

[27] S. Fomin and A. Zelevinsky, Cluster algebras. IV. Coefficients, Compos. Math. 143, 112 (2007).

[28] T. Nakanishi and S. Stella, Diagrammatic description of $c$ vectors and $d$-vectors of cluster algebras of finite type, Electron. J. Comb. 21, 107 (2014).

[29] V. Fock and A. Goncharov, Moduli spaces of local systems and higher Teichmüller theory, Publ. Math. Inst. Hautes Études Sci. 103, 1 (2006).

[30] V. V. Fock and A. B. Goncharov, Cluster ensembles, quantization and the dilogarithm, Ann. Sci. Éc. Norm. Supér. Série 4 42, 865 (2009). 
[31] D. R. Morrison and M. R. Plesser, Nonspherical horizons. 1., Adv. Theor. Math. Phys. 3, 1 (1999).

[32] C. Beasley, B. R. Greene, C. Lazaroiu, and M. Plesser, D3branes on partial resolutions of Abelian quotient singularities of Calabi-Yau threefolds, Nucl. Phys. B566, 599 (2000).

[33] B. Feng, A. Hanany, and Y.-H. He, D-brane gauge theories from toric singularities and toric duality, Nucl. Phys. B595, 165 (2001).

[34] B. Feng, A. Hanany, and Y.-H. He, Phase structure of D-brane gauge theories and toric duality, J. High Energy Phys. 08 (2001) 040.

[35] S. Franco, A. Hanany, K. D. Kennaway, D. Vegh, and B. Wecht, Brane dimers and quiver gauge theories, J. High Energy Phys. 01 (2006) 096.

[36] S. Franco, A. Hanany, D. Martelli, J. Sparks, D. Vegh, and B. Wecht, Gauge theories from toric geometry and brane tilings, J. High Energy Phys. 01 (2006) 128.

[37] K. A. Intriligator and N. Seiberg, Lectures on supersymmetric gauge theories and electric-magnetic duality, Nucl. Phys. B, Proc. Suppl. 45, 1 (1996).

[38] A. Signer, ABC of SUSY, J. Phys. G 36, 073002 (2009).

[39] E. Witten, Phases of $\mathrm{N}=2$ theories in two dimensions, Nucl. Phys. B403, 159 (1993).

[40] H. Garcia-Compean and A. M. Uranga, Brane box realization of chiral gauge theories in two-dimensions, Nucl. Phys. B539, 329 (1999).

[41] K. A. Intriligator, RG fixed points in six-dimensions via branes at orbifold singularities, Nucl. Phys. B496, 177 (1997).

[42] J. D. Blum and K. A. Intriligator, New phases of string theory and 6-D RG fixed points via branes at orbifold singularities, Nucl. Phys. B506, 199 (1997).

[43] K. A. Intriligator, New string theories in six-dimensions via branes at orbifold singularities, Adv. Theor. Math. Phys. 1, 271 (1998).

[44] I. Brunner and A. Karch, Branes at orbifolds versus Hanany Witten in six-dimensions, J. High Energy Phys. 03 (1998) 003.

[45] M. Reid, La correspondance de McKay, Astérisque 276, 53 (2002).
[46] M. Honda and Y. Yoshida, Supersymmetric index on $T^{2} \times$ $S^{2}$ and elliptic genus, arXiv:1504.04355.

[47] A. Gadde, S. S. Razamat, and B. Willett, On the reduction of 4d $\mathcal{N}=1$ theories on $\mathbb{S}^{2}$, J. High Energy Phys. 11 (2015) 163.

[48] B. Feng, Y.-H. He, K. D. Kennaway, and C. Vafa, Dimer models from mirror symmetry and quivering amoebae, Adv. Theor. Math. Phys. 12, 489 (2008).

[49] M. Futaki and K. Ueda, Tropical coamoeba and torusequivariant homological mirror symmetry for the projective space, Commun. Math. Phys. 332, 53 (2014).

[50] K. Hori and C. Vafa, Mirror symmetry, arXiv:hep-th/ 0002222.

[51] K. Hori, A. Iqbal, and C. Vafa, D-branes and mirror symmetry, arXiv:hep-th/0005247.

[52] C. Closset, S. Franco, J. Guo, A. Hasan, and E. Sharpe, Bbranes, Calabi-Yau singularities and graded quivers (to be published).

[53] A. Hanany and D. Vegh, Quivers, tilings, branes and rhombi, J. High Energy Phys. 10 (2007) 029.

[54] D. Kutasov, A Comment on duality in $N=1$ supersymmetric nonAbelian gauge theories, Phys. Lett. B 351, 230 (1995).

[55] D. Kutasov and A. Schwimmer, On duality in supersymmetric Yang-Mills theory, Phys. Lett. B 354, 315 (1995).

[56] D. Kutasov, A. Schwimmer, and N. Seiberg, Chiral rings, singularity theory and electric-magnetic duality, Nucl. Phys. B459, 455 (1996).

[57] G. Musiker (to be published).

[58] A. King and Y. Qiu, Exchange graphs and Ext quivers, Adv. Math. 285, 1106 (2015).

[59] P. Caldero and F. Chapoton, Cluster algebras as Hall algebras of quiver representations, Commentarii mathematici Helvetici 81, 595 (2006).

[60] A. B. Buan, R. Marsh, M. Reineke, I. Reiten, and G. Todorov, Tilting theory and cluster combinatorics, Adv. Math. 204, 572 (2006).

[61] A. B. Buan, I. Reiten, and H. Thomas, Three kinds of mutation, Journal of algebra 339, 97 (2011). 